\title{
The Effect of Reaction on
}

\section{Compressor Performance}

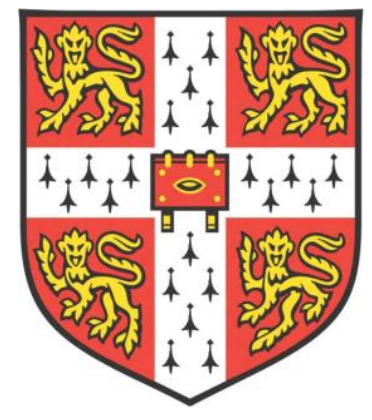

\section{Krishan S Chana}

Department of Engineering

University of Cambridge

This dissertation is submitted for the degree of Doctor of Philosophy 



\section{Declaration}

I hereby declare that except where specific reference is made to the work of others, the contents of this dissertation are original and have not been submitted in whole or in part for consideration for any other degree or qualification in this, or any other university. This dissertation is my own work and contains nothing which is the outcome of work done in collaboration with others, except as specified in the text and Acknowledgements. This dissertation contains fewer than 65,000 words including appendices, references, footnotes, tables and equations and has fewer than 150 figures.

Krishan S Chana

September 2020 



\section{Acknowledgements}

The author would like to thank a number of people who have supported the work in this thesis. Most importantly Rob Miller, whose supervision of the work has been invaluable. His enthusiasm has elicited many memorable discussions, which have shaped the work over time. The author is grateful for the freedom entrusted to him to develop his ideas.

The author would also like to thank John Denton for sharing his thoughts on the work. His flair as a compressor designer has been particularly inspirational. In addition, the calculations in this Thesis would not have been possible without his program, TBlock.

Discussions with Nick Cumpsty and Simon Gallimore have provided insight into the impact of the work on compressor design. Chris Hall, from Rolls-Royce Derby, has supported the work from the start. His trust has allowed the work to develop naturally.

The support of John Denton, Tom Hynes and John Adamczyk was critical in the early development of the 'rotation model', for which the author is very thankful.

The Whittle Laboratory has been a wonderful environment to work in. It has been a privilege to be part of such an encouraging community. The technical expertise of Tony Dickens, James Taylor and Ho-On To have been particularly encouraging.

It has been a privilege to have the opportunity to follow on from the work of Roy Smith, whose life work in engineering has been inspiring to myself and many others.

The author would like to thank Stuart Rankin and his team at the University of Cambridge High Performance Computing Service for providing their technical support. In addition, Rolls-Royce plc. and EPSRC for funding this work. As well as Homerton College for funding my attendance to the annual ASME Turbo Expo conferences. This has given me the chance to meet the broader international gas turbine community.

Finally, I would like to thank my friends and family, whom I love dearly. None of the work in this thesis would have been possible without their support. 



\begin{abstract}
Reaction is the fundamental parameter by which the asymmetry of the velocity triangle of a stage is set. Little is understood about the effect that reaction has on either the efficiency or the operating range of a compressor. A particular difficulty in understanding the effect of reaction is that the rotor and stator have a natural asymmetry caused by the centrifugal effects in the rotor boundary layer, being much larger than those in the stator boundary layer.

In the thesis a novel approach has been taken: McKenzie's 'linear repeating stage' concept is used to remove the centrifugal force effects. The centrifugal effects are then reintroduced as a body force. This allows the velocity triangle effect and centrifugal effect to be decoupled. The ability to accurately decouple these two asymmetries has led to a number of major findings.

The thesis shows the surprising result that, depending on how the solidity of the stage is set, $50 \%$ reaction can either result in the maximum, or the minimum, profile loss. When the solidity is set by the shape factor of the suction-surface boundary layer at the blade trailing-edge, and conventional levels of design work coefficient $\left(\Psi_{d}=0.44\right)$ and flow coefficient $\left(\Phi_{\mathrm{d}}=0.60\right)$ are set, the profile loss becomes independent of reaction.

When the centrifugal effects are removed, $50 \%$ reaction is shown to minimise endwall loss, maximise stage efficiency and maximise operating range. When the centrifugal effects are reintroduced, the compressor with the maximum design efficiency is found to rise in reaction by $5 \%$ (from $50 \%$ reaction to $55 \%$ reaction) and the compressor with the maximum operating range is found to rise in reaction by $15 \%$ (from $50 \%$ reaction to $65 \%$ reaction).
\end{abstract}

In a real multistage compressor there is often a requirement for axial flow at the inlet and exit the compressor. This naturally results in high reaction. In the central stages 
of the compressor, it is possible to maximise the stage efficiency by reducing the reaction to $55 \%$. This is done by raising the interstage swirl through the first stage and dropping it through the last stage. It is shown that if a 10 stage compressor, which originally had a constant stage reaction of $75 \%$, was rebladed so that the central 8 stages had $55 \%$ reaction, then the overall design efficiency would rise by $0.58 \%$. 


\section{Table of contents}

List of figures $\quad$ xiii

Nomenclature $\quad$ xix

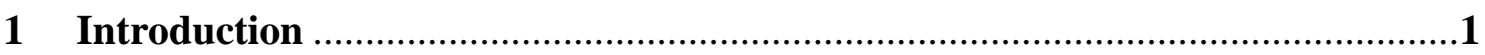

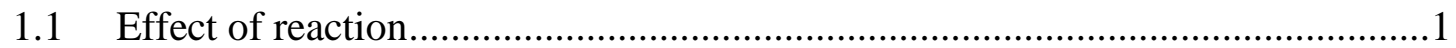

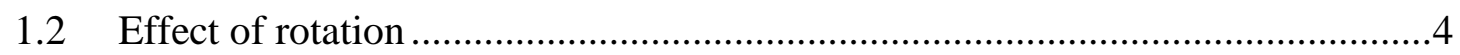

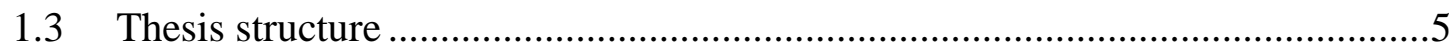

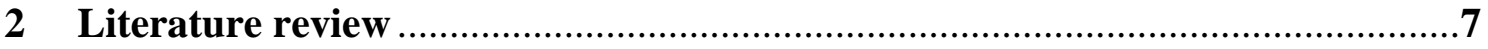

2.1 Effect of reaction on compressor performance ............................................

2.1.1 Design performance of the $50 \%$ reaction stage ......................................

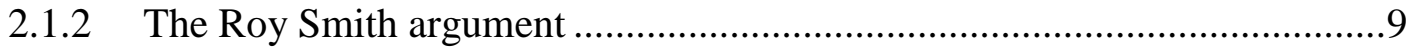

2.1.3 Parametric studies of reaction .................................................................. 10

2.1.4 Influence of reaction across the Smith Chart ............................................14

2.1.5 Impact of reaction on endwall boundary layer re-energisation...................15

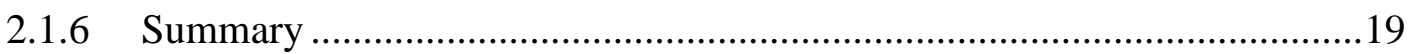

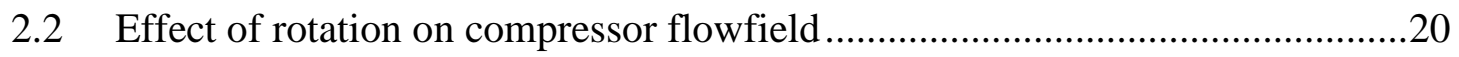

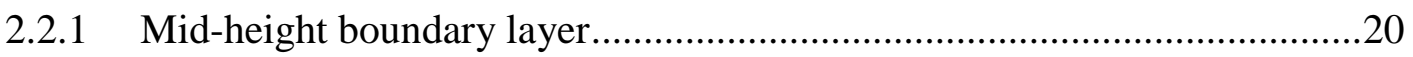

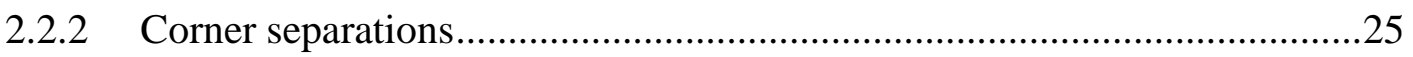

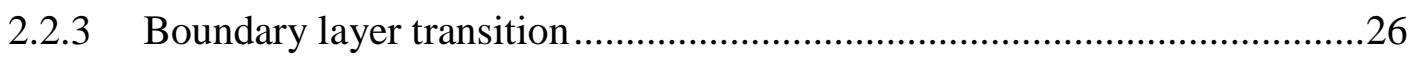

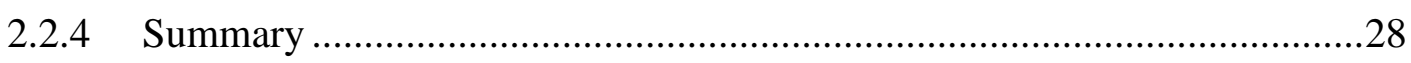

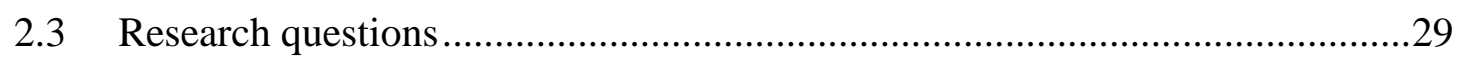

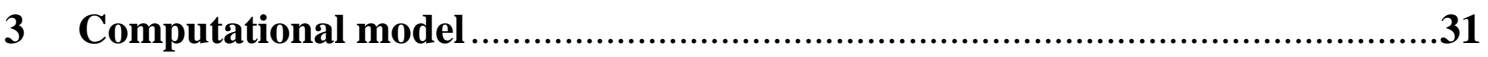

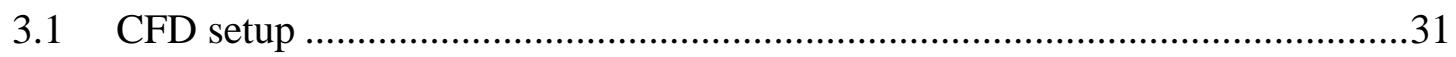

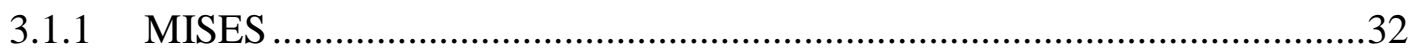

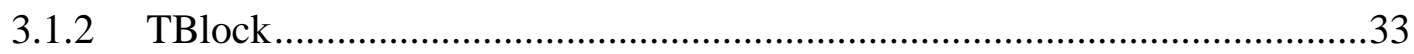




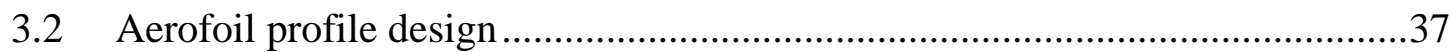

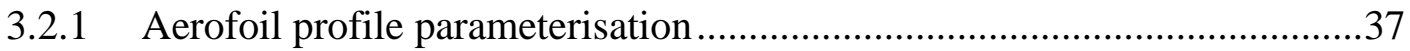

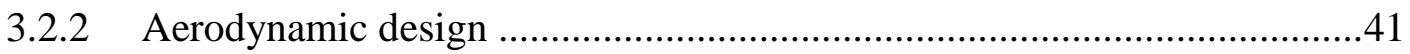

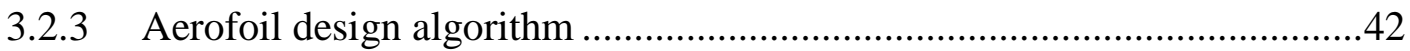

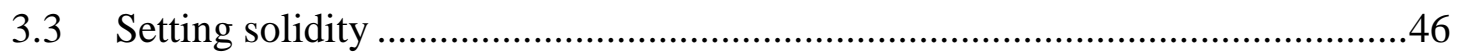

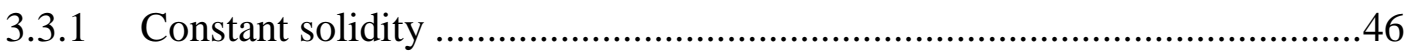

3.3.2 Solidity set by equivalent diffusion ratio ................................................47

3.3.3 Solidity set by trailing-edge shape factor................................................48

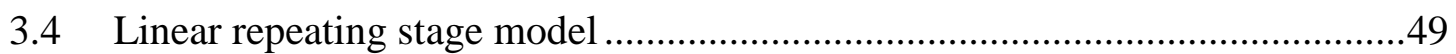

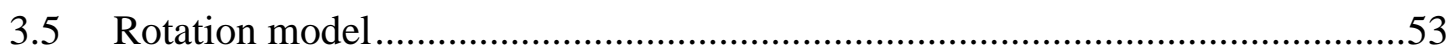

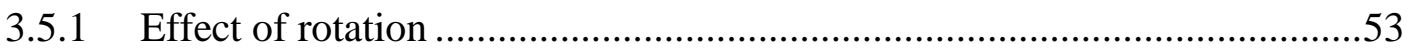

3.5.2 Reintroduction of differential rotational forces........................................54

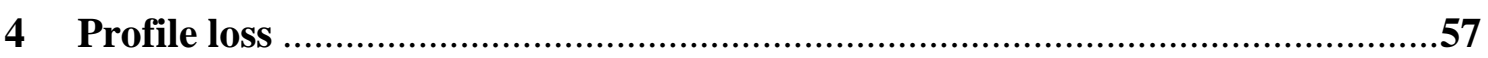

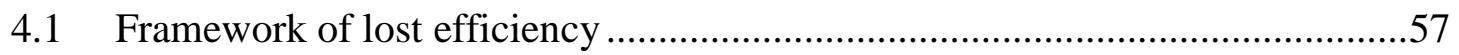

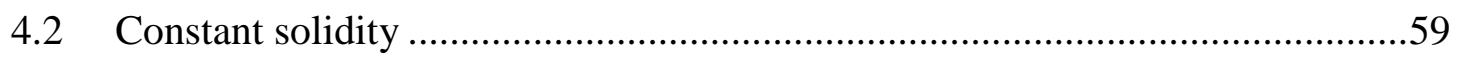

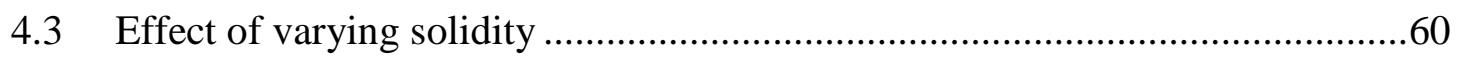

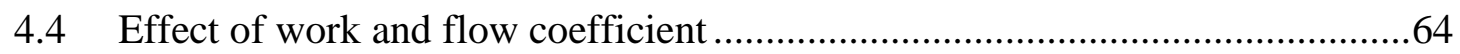

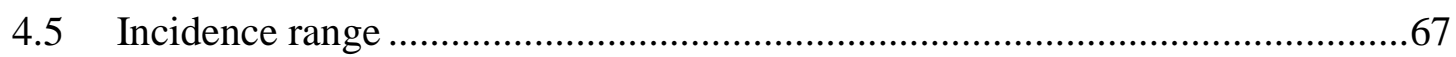

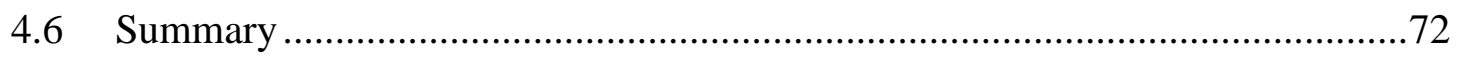

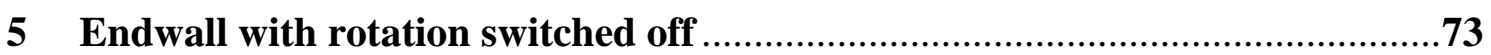

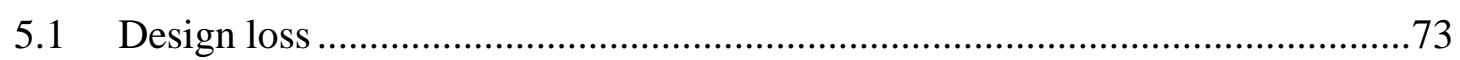

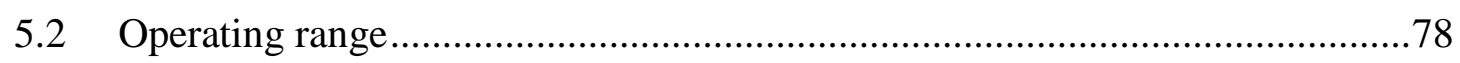

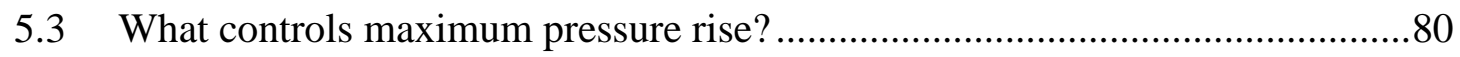

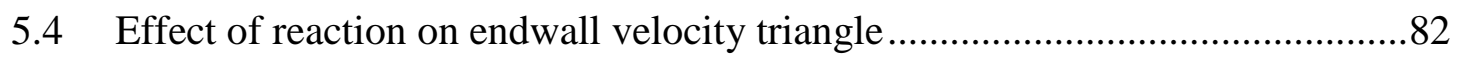

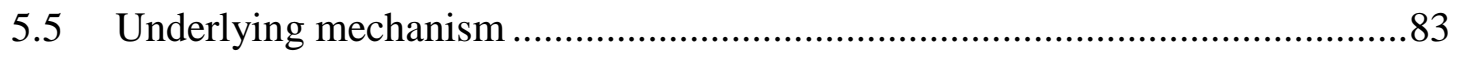

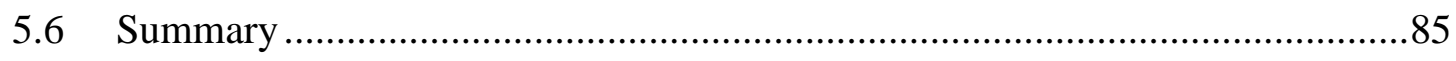

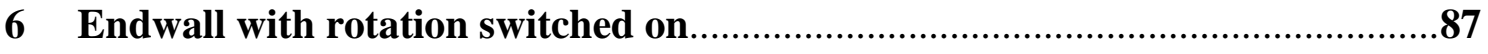

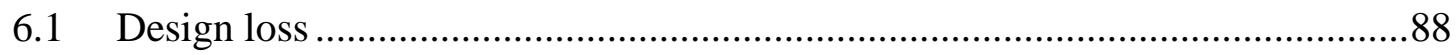

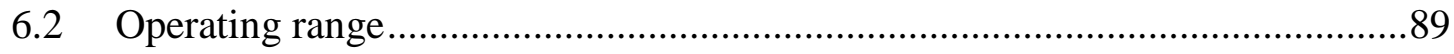

6.3 Why does rotation extend operating range?.................................................92

6.4 Why are rotation effects larger in the rotor than in the stator? .........................94

6.5 Why does rotation extend operating range further at high reaction? .................96 


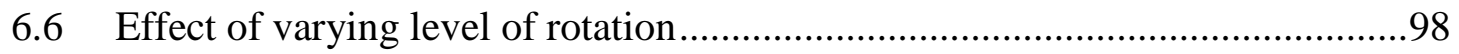

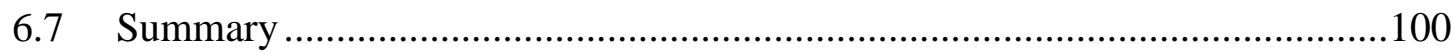

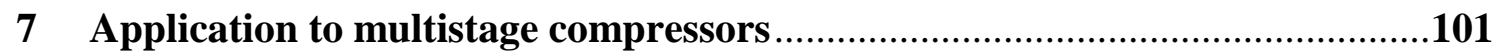

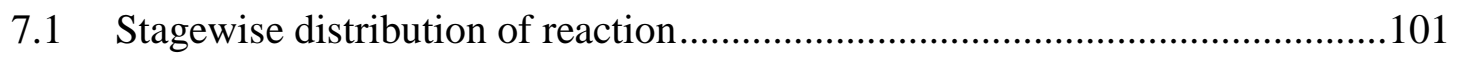

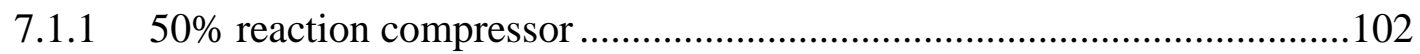

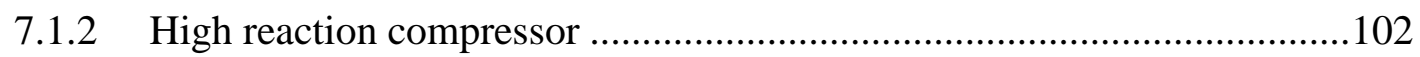

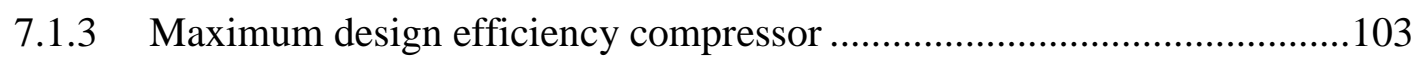

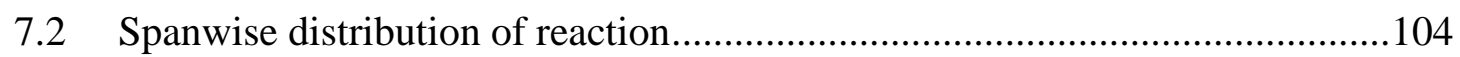

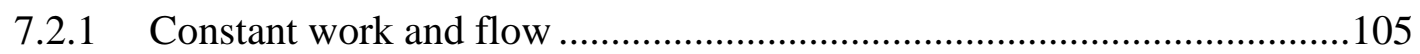

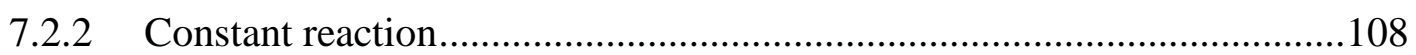

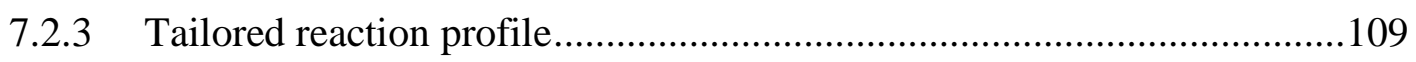

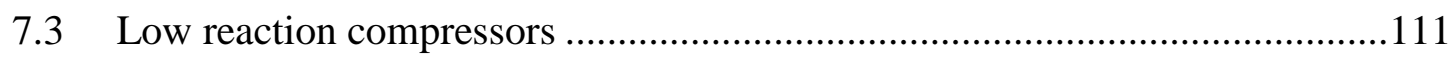

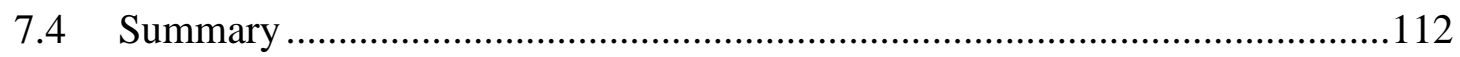

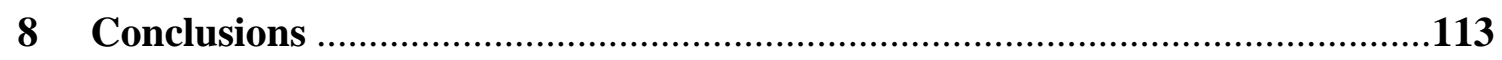

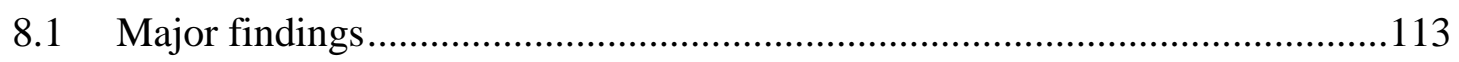

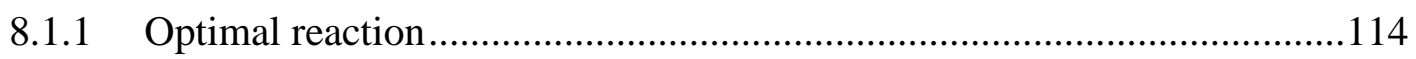

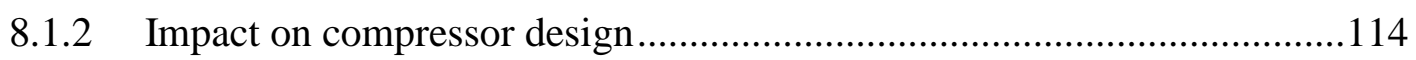

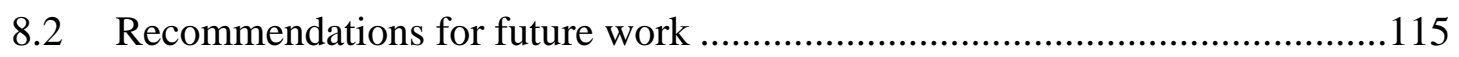

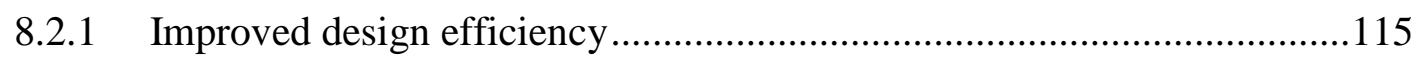

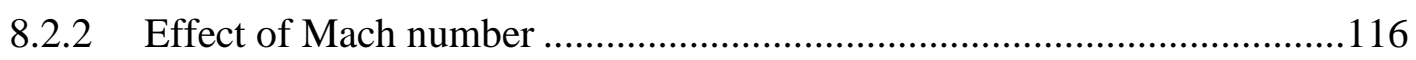

8.2.3 Exploiting rotation effects in 3-D rotor design ......................................116

$\begin{array}{ll}\text { References } & 117\end{array}$

$\begin{array}{lll}\text { Appendix A Low order endwall loss model } & 123\end{array}$

$\begin{array}{lll}\text { Appendix B Derivation of radial equilibrium equation } & 125\end{array}$ 



\section{List of figures}

1.1 Effect of reaction on velocity triangle asymmetry. 50\% reaction (left) and 70\% reaction (right)

1.2 Effect of reaction on aerofoil profile stagger. LHS: rotor and RHS: stator. .3

1.3 Two effects of rotation: (1) bulk passage effect; and (2) differential boundary layer effect

2.1 Rotor inlet (left) and stator inlet (right) velocity triangles for a 50\% (black) and $70 \%$ (red) reaction compressor.

2.2 Estimated variation in lost efficiency based on profile loss, with reaction. Adapted from Cumpsty (1987) ......

2.3 Variation of stage lost efficiency with reaction, produced using data from Dickens \& Day (2011).

2.4 Comparison of $50 \%$ reaction (dotted) to high reaction (solid) on efficiency and operating range. Adapted from Horlock (1958)....

2.5 Smith chart for 50\% reaction (top) and 90\% reaction (bottom) compressor.

Adapted from Casey (1987)

2.6 Variation of stalling static-pressure rise coefficient with stagger angle, taken from Koch (1981)

2.7 Definition of the minimum velocity $V_{\min }$ in the determination of the "effective dynamic pressure factor' $F_{e f}$, for an example velocity triangle design. Adapted from Koch (1981)

2.8 Variation of stalling static-pressure rise coefficient with 'effective dynamic pressure factor' $F_{\text {ef }}$, taken from Koch (1981).....

2.9 Suction-surface limiting streamlines for $50 \%$ reaction, illustrating the asymmetry between loss mechanisms in the rotor and the stator.....

2.10 LHS: Schematic of blade boundary layer (red) and freestream streamline (black) in the meridional view, in the rotor. RHS: Blade boundary layer (red) and freestream velocity triangle (black) 
2.11 Surface limiting streamlines on rotor blade suction-surface (left) and pressuresurface (right). Adapted from Zierke (1994a)

2.12 Corner separation flow topology of rotor (left) and stator (right). Adapted from Taylor (2019)

2.13 Isosurfaces of $\lambda_{2}$-criterion coloured by spanwise velocity showing the pressure-side leg of the horseshoe vortex with the Coriolis force activated (left) and switched off (right), taken from Baum et al. (2016)

2.14 Schematic illustrating a vortex aligned with the axis of rotation and the direction of the associated Coriolis force for the case with rotation (left) and opposite rotation

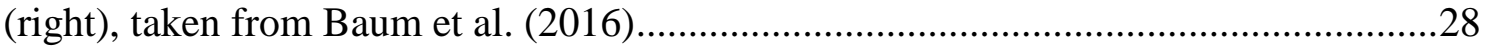

3.1 Example grid produced by MISES for 10\% reaction rotor …................................32

3.2 Example grid produced by Tblock for 50\% reaction stage ...................................34

3.3 Example first grid layer produced by Tblock for 50\% reaction stage.....................35

3.4 Compressor design in meridional (top) and blade-to-blade view (bottom).............36

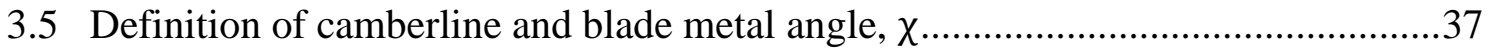

3.6 Non-dimensional blade metal angle distribution for $10 \%$ reaction rotor .................39

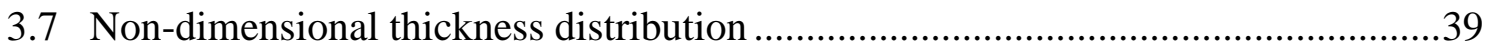

3.8 Non-dimensional transformed thickness distribution ..........................................40

3.9 Definition of 'smooth flow' condition (left) and local incidence (right) ................42

3.10 Flow chart of setting blade inlet and exit metal angles .......................................44

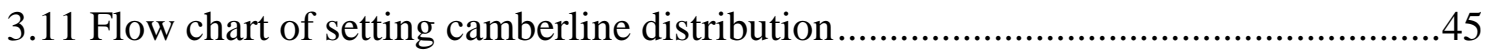

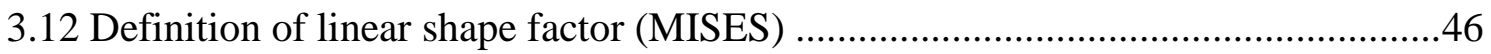

3.13 Flow chart of setting solidity based on constant trailing-edge shape factor............48

3.14 Experimental measurements from 12-stage compressor, taken from Smith (1970)

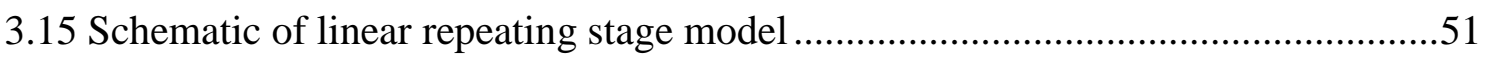

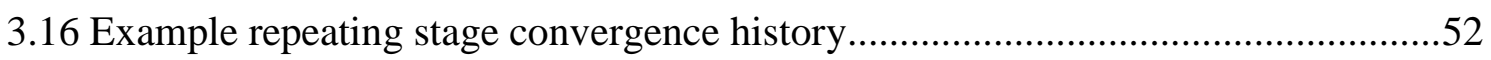

3.17 Two effects of rotation: (1) bulk passage effect; and (2) differential boundary layer

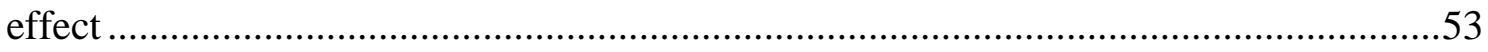

3.18 Real compressor (left) and compressor cascade (right) with rotation model ..........54

3.19 Example perturbation centrifugal force calculation ............................................55

4.1 Blade row lost efficiency for the case of constant solidity and blade loss coefficient

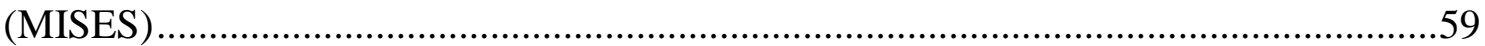

4.2 Stage lost efficiency for the cases of constant solidity, $\mathrm{D}_{\mathrm{eq}}^{*}$ and $\mathrm{H}_{\mathrm{te}}$ (MISES) ......60 
4.3 Reduction in solidity relative to $50 \%$ reaction for the cases of constant $\mathrm{D}_{\text {eq }}^{*}$ and $\mathrm{H}_{\text {te }}$ (MISES). .61

4.4 Change in term 1 (equation 4.7) and term 2 (equation 4.8) relative to 50\% reaction for the rotor.

4.5 Change in term 1 (equation 4.7) and term 2 (equation 4.8) relative to $50 \%$ reaction for the stator.

4.6 Smith Chart showing the effect of reaction on efficiency for the case of constant solidity

4.7 Smith Chart showing the effect of reaction on efficiency for the case of constant $\mathrm{D}_{\mathrm{eq}}^{*}$

4.8 Variation of stage lost efficiency with reaction at points A, B and C in Figures 4.6 and 4.7

4.9 Change in suction-surface trailing edge shape factor relative to $50 \%$ reaction for the cases of constant solidity and $\mathrm{D}_{\mathrm{eq}}^{*}$ (MISES).

4.10 Rotor lost efficiency loop showing the effect of reaction on incidence range for the case of constant $\mathrm{H}_{\mathrm{te}}$ (MISES). Line increment: 0.1 ...... .68

4.11 Definition of negative incidence (left) and positive incidence (right)

4.12 Stator lost efficiency loop showing the effect of reaction on incidence range for the case of constant $\mathrm{H}_{\text {te }}$ (MISES). Line increment: 0.1

4.13 Lost efficiency loop showing the effect of reaction on rotor incidence range for the case of constant solidity (MISES). Line increment: 0.1 .70

4.14 Lost efficiency loop showing the effect of reaction on rotor incidence range for the

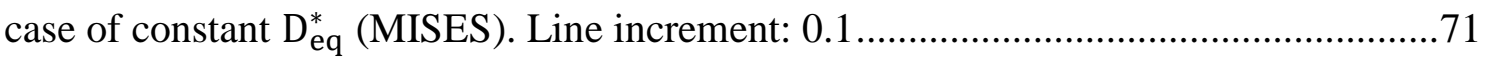

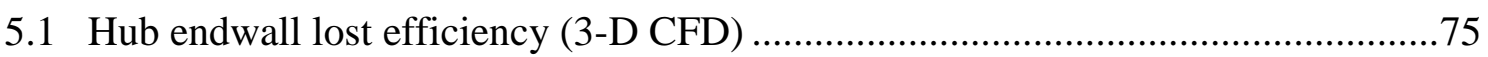

5.2 Effect of reaction on velocity triangle asymmetry. 50\% reaction (left) and $70 \%$ reaction (right)

5.3 Mid-span streamline relative to hub for 30\% reaction (left) and $70 \%$ reaction

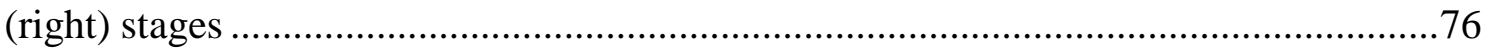

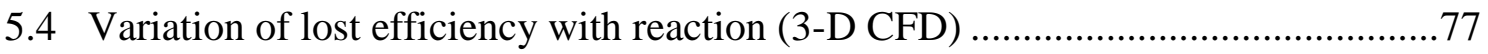

5.5 Static-pressure rise characteristics for compressors without clearances (3-D CFD)

5.6 Static-pressure rise characteristics for compressors with clearances (3-D CFD)....79

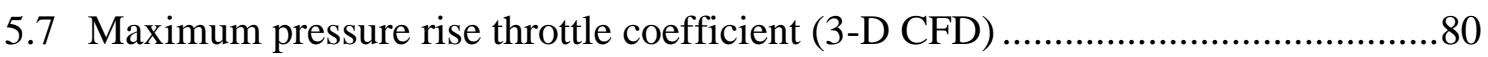

5.8 Stator suction-surface limiting streamlines for the case of zero clearances (3-D CFD) 
5.9 Spanwise variation in stator static-pressure rise coefficient with zero clearance (3-

D CFD)

5.10 Stator inlet velocity tringles for hub endwall (solid) and freestream (dashed) regions

5.11 Decomposition of the flow into freestream (left) and hub endwall (right)

5.12 Total-pressure rise (left) and work coefficient (right) characteristics for hub endwall region

5.13 Mechanism of maximum pressure rise reduction

6.1 Change in lost efficiency for cases with rotation switched on and off (3-D CFD) 88

6.2 Static-pressure rise characteristics for compressors without clearances and rotation on (3-D CFD)

6.3 Static-pressure rise characteristics for compressors with clearances and rotation on

(3-D CFD)

6.4 Throttle coefficients for cases with zero clearances and rotation switched on and off (3-D CFD).

6.5 Throttle coefficients for cases with $1 \%$ clearances and rotation switched on and off (3-D CFD)

6.6 Suction-surface limiting streamlines for $50 \%$ reaction with rotation switched off (3-D CFD)

6.7 Suction-surface limiting streamlines for 50\% reaction with rotation switched on (3D CFD)

6.8 Differential boundary layer effect of rotation for 50\% reaction (3-D CFD) ..........96

6.9 Differential boundary layer effect of rotation (3-D CFD) ....................................96

6.10 Stator suction-surface limiting streamlines for $70 \%$ reaction without clearances (3-

D CFD)

6.11 Spanwise variation of stator static-pressure rise for case of $70 \%$ reaction (3-D CFD)

6.12 Mechanism rotational effects increase the maximum pressure rise of a stage ........98

6.13 Lost efficiency for cases with varying level of rotation (3-D CFD) ......................99

6.14 Throttle coefficients for cases with varying level of rotation (3-D CFD)

7.1 Comparison of three stagewise distributions of reaction for a 10 stage compressor

7.2 Spanwise variation in reaction for a free vortex design (equation 7.6), where meanline reaction is set to $55 \%$

7.3 Spanwise variation in reaction for a free vortex design (equation 7.6), where hub reaction is set to $65 \%$ 
7.4 Spanwise variation in reaction for $\mathrm{n}=0$ design (equation 7.12 ), where meanline reaction is set to 55

7.5 Mid-span streamline in absolute frame of reference for $30 \%$ reaction (left) and $70 \%$ reaction (right) stages .

B.1 Components of acceleration for a small fluid element on an axisymmetric path..126 



\section{Nomenclature}

$$
\begin{aligned}
& \text { Symbols } \\
& \mathrm{c}=\text { chord } \\
& \mathrm{k}=\text { throttle coefficient } \\
& \mathrm{p}=\text { pressure } \\
& \mathrm{r}=\text { effective radius } \\
& \mathrm{t}=\text { blade maximum thickness } \\
& \mathrm{T}=\text { temperature } \\
& \mathrm{U}=\text { rotor blade speed } \\
& \mathrm{V}=\text { absolute velocity } \\
& \mathrm{W}=\text { relative velocity } \\
& \Delta \mathrm{h}_{0}=\text { change in specific stagnation enthalpy } \\
& \Delta \mathrm{r}=\text { blade span } \\
& \Delta \mathrm{s}=\text { change in specific entropy } \\
& \mathrm{AR}=\text { aspect ratio } \\
& \mathrm{C}_{\mathrm{d}}=\text { dissipation coefficient } \\
& \mathrm{C}_{0}=\text { isentropic stage reference velocity } \\
& \mathrm{C}_{\mathrm{p}}=\text { local static-pressure rise coefficient } \\
& \mathrm{D}_{\mathrm{eq}}^{*}=\text { equivalent diffusion ratio } \\
& \mathrm{DF}=\text { diffusion factor } \\
& \mathrm{DH}=\text { de Haller number } \\
& \mathrm{F}_{\mathrm{c}}=\text { perturbation centrifugal force }
\end{aligned}
$$




$$
\begin{aligned}
\mathrm{F}_{\mathrm{r}} & =\text { radial force } \\
\mathrm{H}_{\mathrm{te}} & =\text { trailing edge boundary layer shape factor } \\
\mathrm{M}_{\mathrm{u}} & =\text { Mach number based on blade speed } \\
\mathrm{Re}_{\mathrm{c}} & =\text { Reynolds number based on chord } \\
\dot{\mathrm{S}} & =\text { entropy creation }
\end{aligned}
$$

\section{Subscripts}

$$
\begin{aligned}
0 & =\text { stagnation } \\
1 & =\text { rotor inlet } \\
2 & =\text { stator inlet } \\
\mathrm{bl} & =\text { boundary layer } \\
\mathrm{d} & =\text { design } \\
\mathrm{fs} & =\text { freestream } \\
\text { in } & =\text { inlet } \\
\mathrm{x} & =\text { axial } \\
\theta & =\text { circumferential }
\end{aligned}
$$

\section{Greek Symbols}

$$
\begin{aligned}
\Phi & =\text { flow coefficient } \\
\Psi & =\text { work coefficient } \\
\Lambda & =\text { reaction } \\
\varepsilon & =\text { clearance } \\
\psi^{\prime} & =\text { static-pressure rise coefficient } \\
\eta & =\text { isentropic efficiency } \\
\sigma & =\text { solidity }
\end{aligned}
$$




\section{Chapter 1}

\section{Introduction}

\subsection{Effect of reaction}

In the central stages of a multistage compressor, it is typically argued that symmetrical rotor and stator velocity triangles maximise the stage efficiency. Horlock (1958) and Cumpsty (1989) say this is because the static-pressure rise is split equally between the rotor and stator and so the adverse pressure gradient is balanced. This is the definition of $50 \%$ reaction, as described by equation 1.1 .

$$
\Lambda=\frac{\Delta \mathrm{p}_{\text {rotor }}}{\Delta \mathrm{p}_{\text {stage }}}
$$

Denton (1993) also argues for symmetrical velocity triangles maximising the stage efficiency, based on balancing the relative inlet velocities into the rotor and stator. Only in a compressor stage of $50 \%$ reaction are the relative inlet velocities into the rotor and stator equal. At any other value of reaction, the relative inlet velocities into the rotor and stator are not equal. As the increase in specific entropy due to the surface boundary layers on each blade is proportional to the cube of the surface velocity, the blade with the increased relative inlet velocity will have a greater increase in specific entropy than the reduction in the other blade. There is therefore a reduction in stage efficiency for asymmetric velocity triangles. 
These views were the views of the author until he received a personal communication from Dr L.H. Smith (LHS) on 15th October 2015:

'I have found that 50\% reaction does not always give the highest efficiency. For a given flow coefficient and work coefficient, 75\% reaction gives higher efficiency than 50\% reaction. This happens because the lower solidities can be used with high reaction blading, holding $D_{e q}^{*}$ constant.'

LHS went on to reference a discussion he wrote for Lieblein's (1959) paper where he used Lieblein's effective diffusion ratio $\mathrm{D}_{\mathrm{eq}}^{*}$ to set the solidity of a stage, and this showed that $50 \%$ reaction did not produce a compressor with the highest design efficiency. There is further evidence for this by Casey (1987) who used a preliminary design system to study the effect of reaction.

In real compressors the reaction is not an independent design variable. This is because at the inlet and exit of a multistage compressor there is zero absolute swirl and this results in high reaction. This corresponds to designs with typically high reactions in the range $70 \%$ to $90 \%$, McKenzie (1997). In the central stages of the compressor, it is possible reduce the reaction to $50 \%$ by raising the interstage swirl through the first few stages and dropping it through the last few stages.

However the reaction is chosen, it is important to understand how it effects the overall efficiency and operating range of the compressor. Figure 1.1 compares the stage velocity triangles for a 50\% (left) and 70\% (right) reaction design, both with equal flow and work coefficient. It illustrates why reaction is the fundamental parameter which sets the asymmetry between the rotor and stator velocity triangles. 


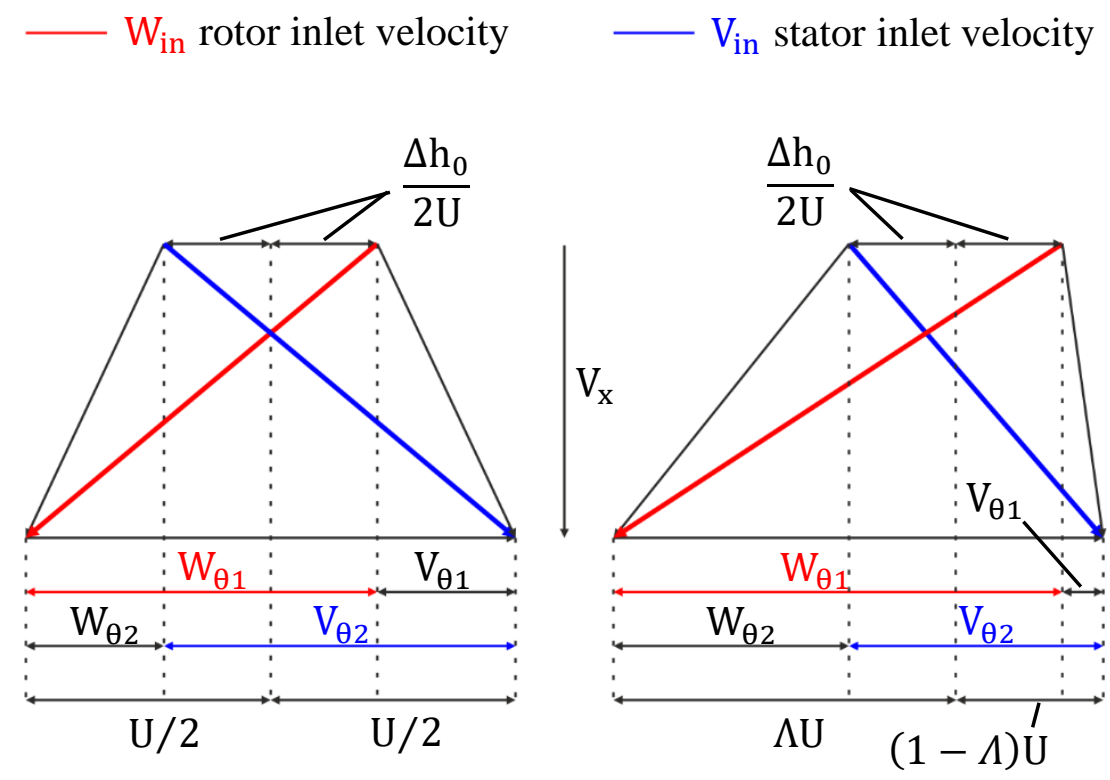

Figure 1.1: Effect of reaction on velocity triangle asymmetry. 50\% reaction (left) and $70 \%$ reaction (right).

As the reaction rises, the rotor inlet velocity $W_{\text {in }}$ rises and the stator inlet velocity $V_{\text {in }}$ drops. It follows that as the reaction rises, the rotor stagger increases and the stator stagger decreases. This is illustrated in Figure 1.2, which compares the aerofoil profiles for the $50 \%$ and $70 \%$ reaction rotors on the left, and the stators on the right. In the case of the $50 \%$ reaction stage, the rotor and stator aerofoil profiles are identical.

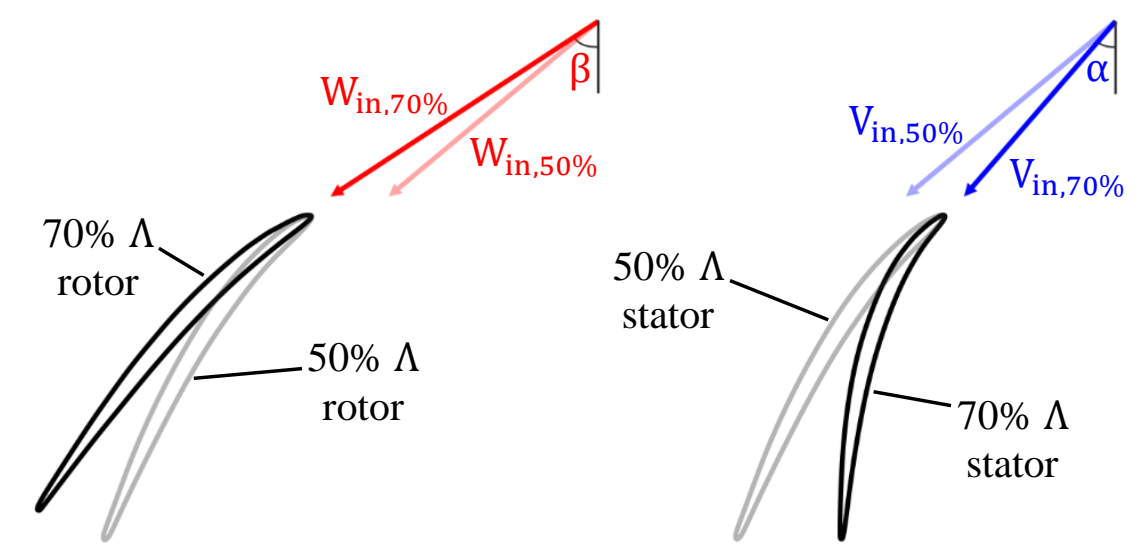

Figure 1.2: Effect of reaction on aerofoil profile stagger. LHS: rotor and RHS: stator. 


\subsection{Effect of rotation}

There is an added natural asymmetry between the rotor and stator caused by rotational forces. This asymmetry is due to two effects of rotation, shown in Figure 1.3. First, a bulk passage effect, where the centrifugal forces in the freestream are balanced by a radial pressure gradient. This causes the bulk passage flow in the rotor to move radially outward and in the stator to move radially inward. Second, a differential boundary layer effect where the differential effect of centrifugal and Coriolis forces cause the boundary layers in the rotor to be accelerated towards the casing, Horlock \& Wordsworth (1965). This effect is critical to this paper as it acts as a natural asymmetry between the way in which the rotor and stator boundary layers develop.

There is some experimental evidence in the literature, Seippel (ref in Horlock (1958)) and Farmakalides et al. (1994), to show that high reaction designs are advantageous due to the presence of rotational forces in the rotor. However, in these studies it is difficult to decouple the effects of reaction on the velocity triangle and on the rotational force in the boundary layer, because they are linked. To overcome this problem a new rotation model has been developed which uses the McKenzie's 'linear repeating stage' concept to first remove the effects of rotation. The differential boundary layer effects of rotation are then reintroduced as a body force, in a controlled way.

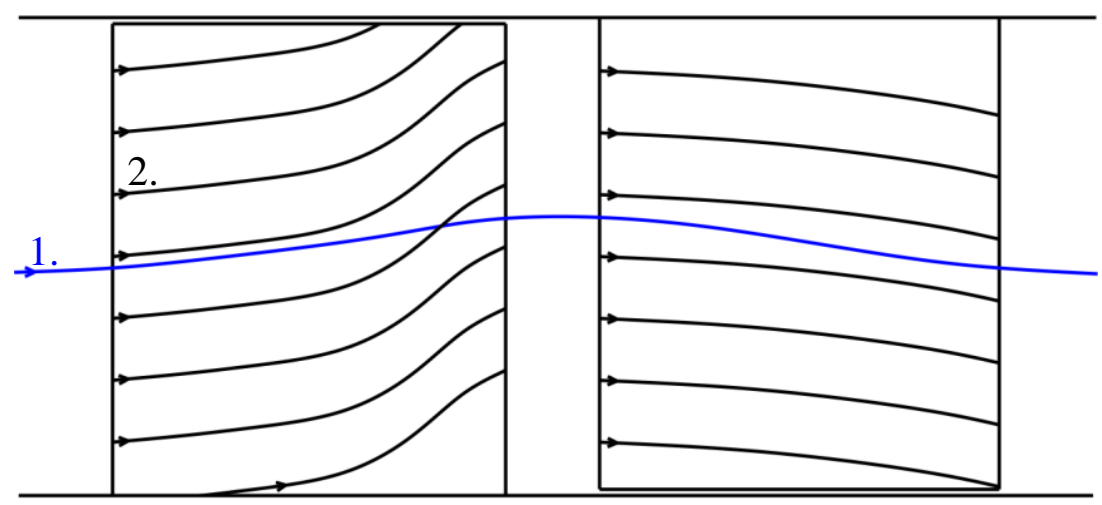

Figure 1.3: Schematic showing two effects of rotation: (1) bulk passage effect on freestream streamline; and (2) differential boundary layer effect on rotor and stator boundary layer streamlines. 
In real compressors the choice of reaction also introduces asymmetry into the stage Mach number triangles. For a constant axial velocity ratio, it is thought that $50 \%$ reaction maximises the stage efficiency based on balancing the relative inlet Mach numbers into the rotor and stator. This becomes more important as the Mach numbers increase due to the increased peak Mach numbers on the rotor and stator and increased shock losses. To decouple the effects of reaction from the effects of Mach number in this study, a blade speed Mach number of 0.3 was chosen so that the flow can be considered incompressible.

The compressor designs presented in this thesis have repeating stages, and as a result, the shape of the design velocity triangle is controlled by fixing the flow coefficient $\Phi$, work coefficient $\Psi$, and reaction $\Lambda$. Our understanding of the effect of the shape of the design velocity triangle on compressor performance has developed for over half a century. Much of this understanding is founded on the measurements observed from the stationary cascade tests of Lieblein (1959) and Koch \& Smith (1976). There is still considerable debate over the effect of reaction on compressor design efficiency and operating range. The thesis shows that the confusion is due in part to the inability of previous work to decouple the effects of the centrifugal force and the effects of changing the velocity triangle, in a tractable way. A unique model has been developed to confront this problem. The model allows the effect of the centrifugal force and the effect of reaction on compressor performance, to be studied independently for the first time. This is the focus of the Thesis.

\subsection{Thesis structure}

A review of the literature is undertaken in Chapter 2 and is followed by a description of the computational model in Chapter 3. The results in this thesis then follow in the subsequent four chapters, in turn. The effect of reaction on profile loss is investigated in Chapter 4. The effect of reaction on the endwall with rotation 'switched off' is investigated in Chapter 5. The effect of reaction on the endwall with rotation 'switched on' is investigated in Chapter 6. We are then in a position to understand how the choice of reaction affects the overall lost efficiency of a multistage core compressor, which is 
discussed in Chapter 7. At the end of this thesis the major findings are summarised and recommendation for future work is made, in Chapter 8. 


\section{Chapter 2}

\section{Literature review}

The literature review is structured in two sections. The effect of reaction on the performance of the compressor and the effect of rotation on the flowfield of the compressor, are examined in turn.

\subsection{Effect of reaction on compressor performance}

This section is further divided into five parts. The first part explains why $50 \%$ reaction is typically thought to maximise stage efficiency. The second looks at why LHS thought that 50\% reaction did not give the most efficient stage. The third reviews the limited number of parametric studies of reaction. The fourth looks at the effect of reaction on the shape of the compressor Smith Chart. The final part looks at how the frame of reference change in a compressor affects endwall loss. This is included in the review as later in the thesis this mechanism will be shown to be important.

\subsubsection{Design performance of the $50 \%$ reaction stage}

In the central stages of a multistage compressor, it is typically argued that symmetrical rotor and stator velocity triangles maximises the stage efficiency. Horlock (1958) and Cumpsty (1989) say this is because the static-pressure rise is split equally between the rotor and stator and therefore the adverse pressure gradient is balanced. 
There is also an argument for symmetrical velocity triangles maximising stage efficiency based on balancing the relative inlet velocities into the rotor and stator. Denton (1993) explains that this is because the relative inlet velocities into the rotor $W_{\text {in }}$ and into the stator $V_{\text {in }}$ are equal. $W_{\text {in }}$ and $V_{\text {in }}$ in this case, are shown as the solid black vectors in the velocity triangles illustrated in the top half of Figure 2.1. At any other value of reaction, the relative inlet velocities into the rotor and stator are not equal. The solid red vectors show the case of $70 \%$ reaction. As the increase in specific entropy due to the surface boundary layers on each blade is proportional to the cube of the surface velocity, the blade with the increased relative inlet velocity will have a greater increase in specific entropy than the reduction in the other blade. There is therefore a reduction in stage efficiency for asymmetric velocity triangles.
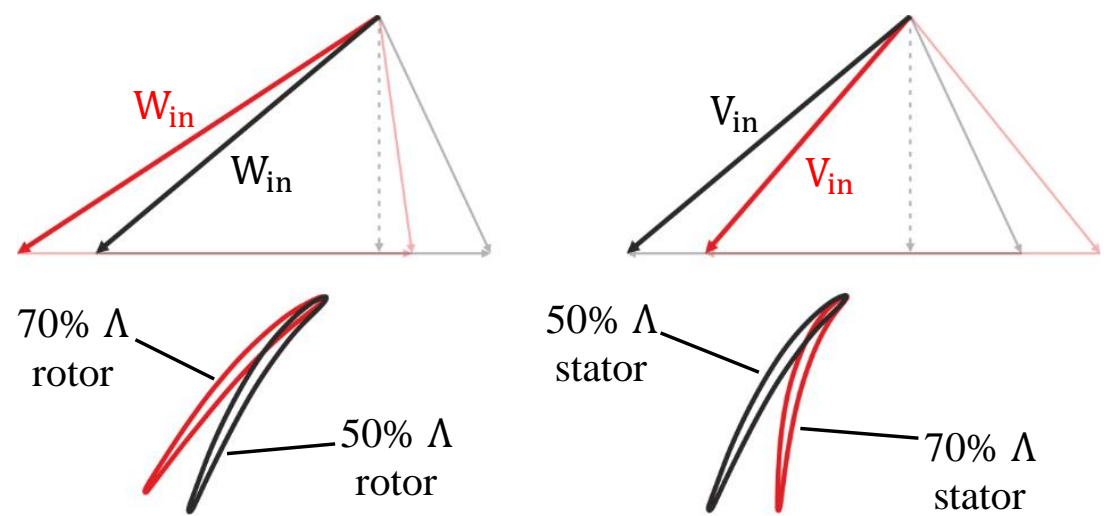

Figure 2.1: Rotor inlet (left) and stator inlet (right) velocity triangles for a 50\% (black) and $70 \%$ (red) reaction compressor.

The corresponding blade shapes to the velocity triangles shown in the top half of Figure 2.1, are shown in the bottom half of Figure 2.1. For the 50\% reaction compressor, the rotor and stator aerofoil profiles are identical. As the reaction rises, $\mathrm{W}_{\mathrm{in}}$ rises, causing the rotor stagger to increase. Simultaneously, $V_{\text {in }}$ drops, causing the stator stagger to decrease.

McKenzie (1997) proposes another argument for symmetrical velocity triangles maximising stage efficiency, again based on balancing the relative inlet velocities into the rotor and stator. First, assume there is a fixed minimum loss coefficient for both blade rows, irrespective of reaction. As the loss in each blade row is proportional to the square 
of the relative velocity into that blade row, there is again a reduction in stage efficiency for asymmetric velocity triangles.

Although Horlock, Cumpsty, Denton and McKenzie employ different arguments, they all agree that $50 \%$ reaction results in the compressor with the highest efficiency.

\subsubsection{The Dr L.H. Smith argument}

The views presented in the previous section were the views of the author until he received a personal communication from Dr L.H. Smith (LHS) on 15th October 2015:

"I have found that 50\% reaction does not always give the highest efficiency. For a given flow coefficient and work coefficient, 75\% reaction gives higher efficiency than $50 \%$ reaction. This happens because the lower solidities can be used with high reaction blading, holding $D_{e q}^{*}$ constant."

LHS went on to reference a discussion he wrote in Lieblein (1959), where he used Lieblein's Effective Diffusion Ratio $\mathrm{D}_{\mathrm{eq}}^{*}$ to set the solidity of a stage and this showed that $50 \%$ reaction did not produce the compressor with the highest design efficiency. To calculate the design efficiencies LHS combined equations 8 and 11 in Lieblein (1959), to estimate the rotor and stator total-pressure loss coefficients $\widetilde{\omega}$. The stage lost efficiency based on profile loss alone can then calculated from:

$$
\frac{\mathrm{T} \Delta \mathrm{s}}{\Delta \mathrm{h}_{0}}=\frac{2\left(\mathrm{~W}_{\text {in,rotor }}^{2} \widetilde{\omega}_{\text {rotor }}+\mathrm{V}_{\text {in,stator }}^{2} \widetilde{\omega}_{\text {stator }}\right)}{\Delta \mathrm{h}_{0}}
$$

Using equation 2.1 with a work coefficient $\Psi$ of 0.35 and a flow coefficient $\Phi$ of 0.55 , the $75 \%$ reaction compressor is approximately $0.43 \%$ more efficient than the $50 \%$ reaction compressor. LHS explained that this is because the solidity of the stage drops. However, he does not explain why the solidity drops in this way.

LHS's argument highlights that the views discussed in the previous section do not include the effect of varying solidity. Although he does not study this effect in detail, his 
study shows that including the effect results in a higher stage efficiency for $75 \%$ reaction, over $50 \%$ reaction.

\subsubsection{Parametric studies of reaction}

A limited number of researchers have studied the effect of systematically varying reaction. These researchers have employed very different approaches. The first two studies presented in this section use loss correlations to study the effect. The second study uses 3-D RANS CFD calculations. The final two studies are based on experimental measurements.

In his parametric study, Cumpsty (1989) uses identical loss correlation for the rotor and stator and uses Lieblein's Diffusion Factor DF to fix the solidity. In contrast to the work of LHS, Cumpsty concluded that the 50\% reaction compressor had the highest efficiency. However, the variation in efficiency with reaction was shown to be relatively weak. For a fixed work coefficient $\Psi$ of 0.4 , flow coefficient $\Phi$ of 0.6 , and Diffusion Factor DF of 0.45 in each blade row, the stage lost efficiency is shown as a function of reaction in Figure 2.2. To calculate the stage lost efficiencies in Figure 2.2, the rotor and stator loss coefficients are estimated using:

$$
\widetilde{\omega}=\frac{0.007}{\cos \left(\alpha_{2}\right)} 2 \sigma
$$

which was shown by Cumpsty to be a good approximation to the cascade data of Lieblein. The stage lost efficiency based on profile loss alone is then calculated from equation 2.1. The $75 \%$ reaction compressor is approximately $0.11 \%$ less efficient than the $50 \%$ reaction compressor in Figure 2.2. Furthermore, the range of reactions over which the efficiency only varies by $0.1 \%$ is relatively wide, between $50 \%$ reaction and $73 \%$ reaction. 


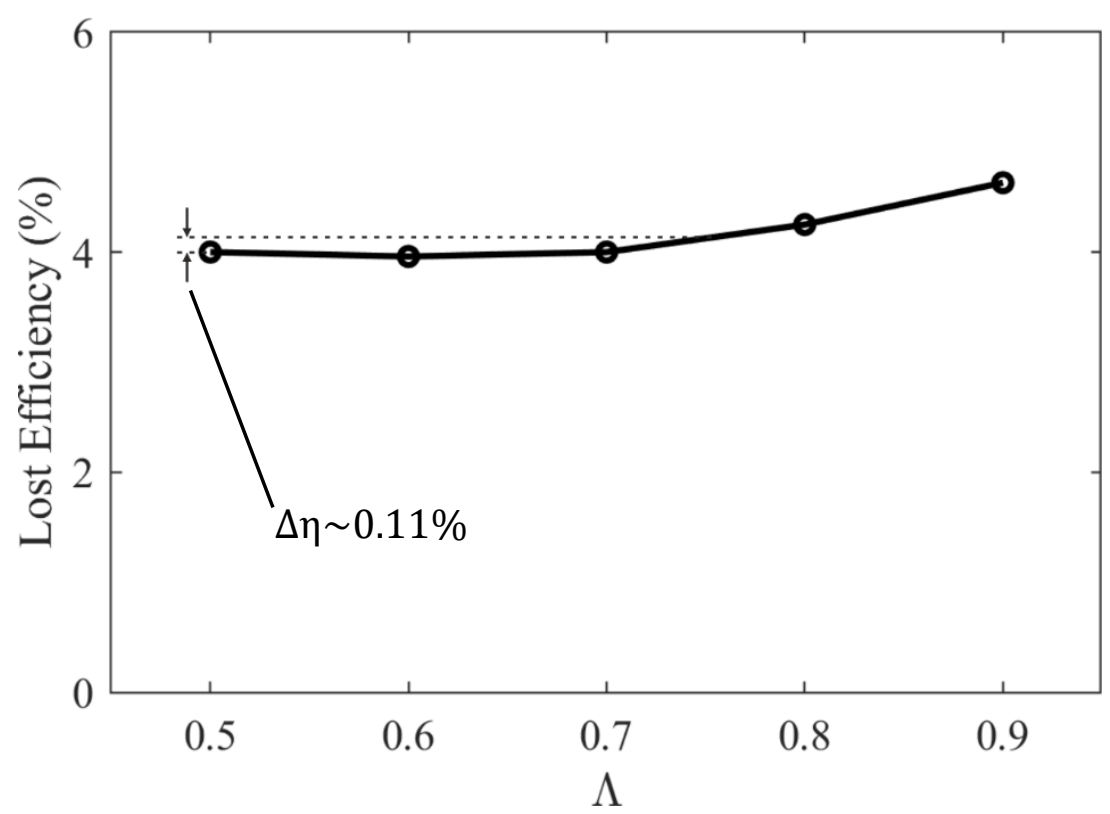

Figure 2.2: Estimated variation in lost efficiency based on profile loss, with reaction. Adapted from Cumpsty (1987).

The parametric study by Casey (1987) includes endwall loss correlations and rotor tip and stator hub clearance models. He concluded that the optimum reaction lies above $50 \%$. The reason for this is that the stage efficiency is largely determined by the endwall and clearance losses, which have different magnitudes in stationary and rotating blade rows. However, he does not explain why this is.

Dickens \& Day (2011) used 3-D RANS CFD calculations to look at the effect of reaction on the lost efficiency of the stage, for three different levels of work coefficient, and a fixed flow coefficient $\Phi$ equal to 0.5 . The solidities were selected to achieve a suction-surface boundary layer trailing edge shape factor $H_{\text {te }}$ in the range 2.1-2.3, calculated using MISES. The results shown in Figure 2.3 have been produced using the data of Dickens \& Day. The blue line shows how at conventional levels of loading $(\Psi=$ $0.45)$, the compressor with highest efficiency has a reaction of approximately $69 \%$. The $50 \%$ compressor has an efficiency which is $1.8 \%$ less than the $69 \%$ compressor. As the work coefficient is increased, the optimum reaction increases, illustrated by the black line in Figure 2.3. 


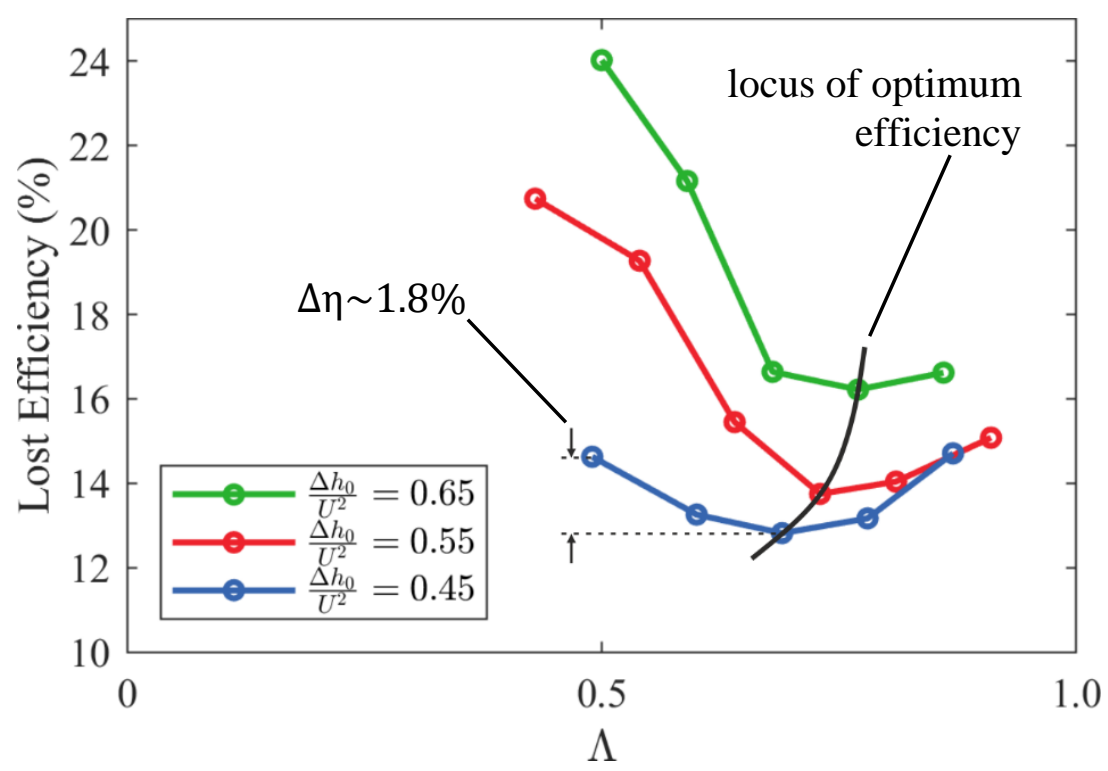

Figure 2.3: Variation of stage lost efficiency with reaction, produced using data from Dickens \& Day (2011).

Dickens \& Day concluded that high reaction is necessary to reduce the static-pressure rise across the stator, as it is more susceptible than the rotor, to large hub corner separations. The difference between the behaviour of the rotor and stator hub corner separations is assumed to arise from the effects of rotation. However, in their study, which is at a hubto-tip ratio of 0.8 , it is difficult to decouple the effects of rotation on the boundary layers and corner separations, from the effects of the changing velocity triangle.

There have been only two experimental studies of reaction. Farmakalides et al. (1994) performed measurements of a low speed, three-stage, axial flow compressor. Their study concluded that the $80 \%$ reaction compressor gave improved stall margin at no decrement in efficiency over $50 \%$ reaction. However, the authors themselves concluded that the different methods employed for the design of the two compressors, could be responsible for the observed improvement in performance. The $80 \%$ reaction was tested at a work coefficient $\Psi$ of 0.4 , flow coefficient $\Phi$ of 0.6 and was designed to a modern standard. Whereas the $50 \%$ reaction was tested at a work coefficient $\Psi$ of 0.326 , flow coefficient $\Phi$ of 0.565 and was designed using circular-arc camberlines. 


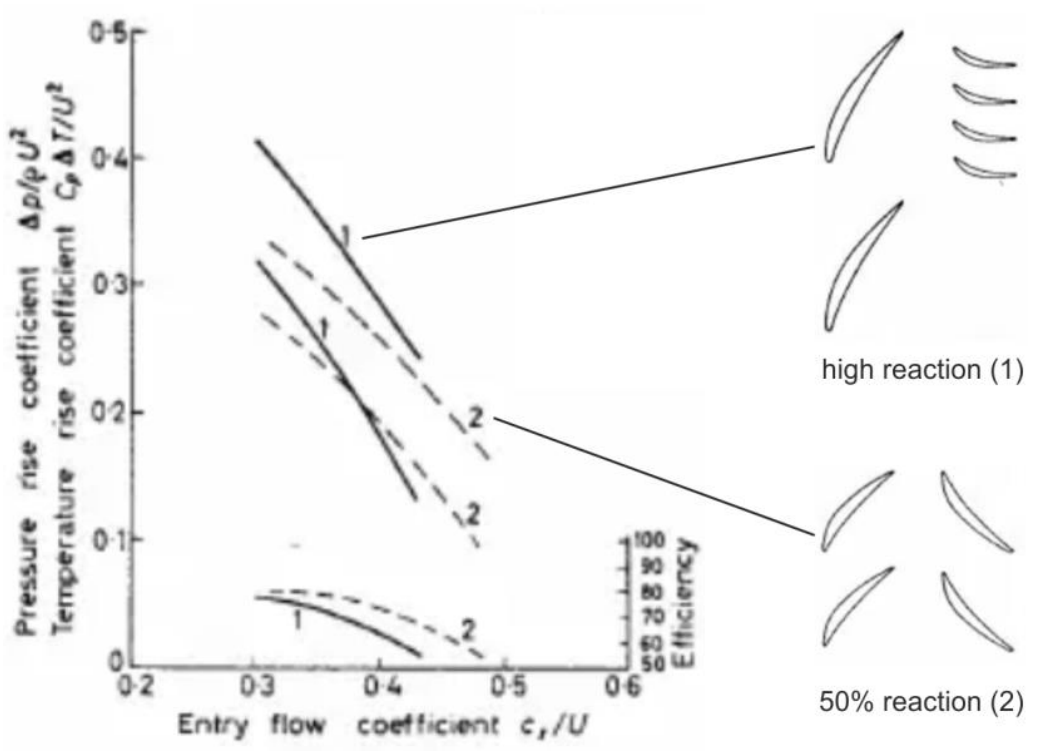

Figure 2.4: Comparison of $50 \%$ reaction (dotted) to high reaction (solid) on efficiency and operating range. Adapted from Horlock (1958).

In the 1950s Seippel conducted experiments to study the effect of reaction on efficiency and operating range. The experiments are referenced in Horlock (1958). Seippel's measurements are displayed in Figure 2.4 which shows characteristics of static-pressure rise, work coefficient and efficiency against flow coefficient for two compressors. The $50 \%$ reaction compressor, shown as the dotted black lines and labelled (2), shows higher efficiencies over the higher reaction compressor, shown as the solid black lines and labelled (1). Seippel concludes that this is because rotating blade rows have a slightly higher efficiency than stationary rows. Seippel goes on to say that this could mean that "an optimum efficiency may occur in the range 50-60\% reaction." However, the resolution of the experiment is too coarse to show this clearly. It is also interesting to note that the higher reaction compressor employs greatly reduced solidities in the rotor compared to the stator. From Figure 2.4 we can estimate that the high reaction rotor has a solidity approximately $45 \%$ less than that of the stator.

In both the experimental studies of reaction, the method and measurement accuracy does not appear to be sufficient to determine whether the observed difference in performance is true. 
It is clear that each of the parametric studies of reaction are limited in some way. The use of correlations does not capture the underlying physics and the experimental results do not have sufficient accuracy. The effect of reaction is captured by the 3-D CFD results of Dickens \& Day, and the results show the asymmetry that rotation introduces. However, the study is limited by the difficulty in decoupling the effects of reaction from other effects.

\subsubsection{Influence of reaction across the Smith Chart}

The Smith Chart shows how the design efficiency of the compressor varies with flow coefficient $\Phi$ and work coefficient $\Psi$. The Smith Chart is typically developed using loss correlations. This makes it subject to the same problems, of not capturing the underlying physics, fixed solidity and no rotational asymmetry, as discussed in the previous section. However, Casey did investigate how reaction changes the shape of the Smith Chart.

The report by Casey (1987) is based on the correlations of Lieblein (1959) and aims to determine the shape of the Smith Chart for a range of reactions. In his study, the solidity is fixed. He concludes that the optimum value of flow and work coefficient leading to the highest efficiency, is a function of reaction. In particular, the optimum value of design work coefficient increases with the design reaction. This can also be seen in the results of Dickens \& Day (2011), shown in Figure 2.3. However, Casey does not explain why this is and does not explain if the same effect is observed as the reaction drops. He also concludes that the widest operating range is obtained at $50 \%$ reaction, although the reasoning for this is not explained.

It is therefore clear that the Smith Chart reported by Casey, which is based on loss correlations, is subject to the same problems as described in the previous section. It does not capture the key physics, the solidity is fixed and no rotation effects are included. 

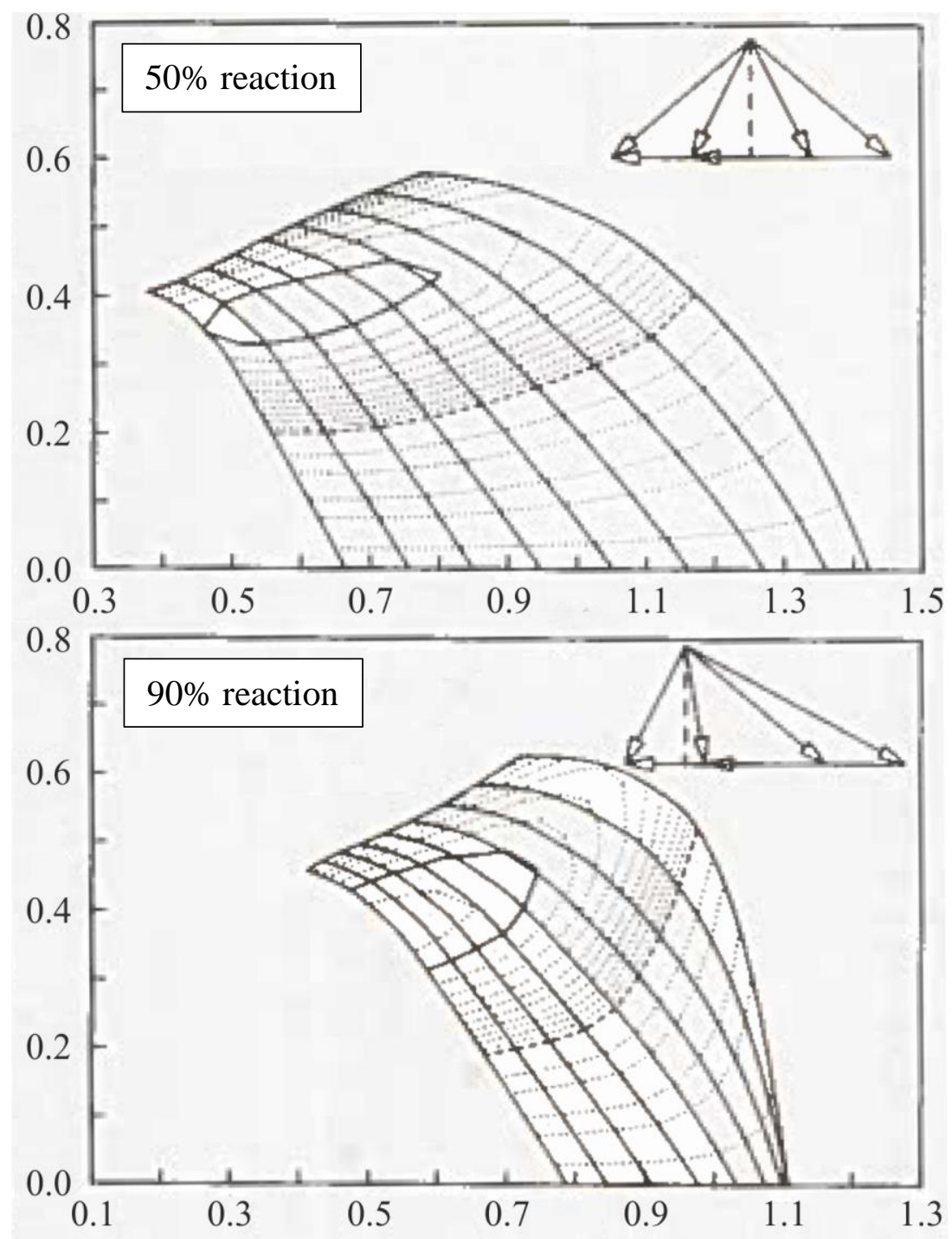

Figure 2.5: Smith chart for 50\% reaction (top) and 90\% reaction (bottom) compressor. Adapted from Casey (1987).

\subsubsection{Impact of reaction on endwall boundary layer re-energisation}

In a multistage stage compressor, one of the most significant mechanisms which changes the development of the endwall boundary layer is the effect of the change of reference frame on the re-energisation of the endwall boundary layer. The effect of reaction on this mechanism has not been studied, but later in the thesis it is shown that this is a critical mechanism, and therefore will be reviewed here. 
Moore \& Richardson (1955) were amongst the earliest to examine the notion of boundary layer re-energisation in axial compressors. They noted that the change of reference frame as the flow travels between the rotor and stator could cause the axial velocity deficit at the exit of the rotor to be re-energised as it entered the stator. Moore \& Richardson first demonstrated that understanding boundary layer re-energisation was critical in determining the stable operating range of a compressor.

The level of boundary layer re-energisation was found by Auchoybur and Miller (2017) to ultimately be limited by the reduction in local static-pressure rise coefficient in the endwall region, $\mathrm{C}_{\mathrm{p}, \mathrm{ew}}$, relative to the freestream, $\mathrm{C}_{\mathrm{p}, \mathrm{fs}}$. In a repeating stage, the staticpressure rise through a bladerow is the same in the endwall and freestream. The ratio of $C_{p, f s}$ to $C_{p, e w}$ is therefore determined by the ratio of endwall to freestream inlet dynamic pressure. This is described by equation 2.4. This ratio of dynamic pressures can therefore be thought of as determining the maximum pressure rise capability of a stage.

$$
\frac{\mathrm{C}_{\mathrm{p}, \mathrm{fs}}}{\mathrm{C}_{\mathrm{p}, \mathrm{ew}}}=\frac{\mathrm{V}_{\mathrm{ew}}^{2}}{\mathrm{~V}_{\mathrm{fs}}^{2}}
$$

The ratio of endwall to freestream inlet dynamic pressure, noted by Auchoybur and Miller to be critical in determining the maximum pressure rise capability of a stage, was uncovered by Koch (1981). Koch studied of a wide range of compressor test measurements, shown in Figure 2.6, and concluded that machines with a relatively high rotor stagger had high stalling static-pressure rise coefficients. The data suggested that high stagger designs would observe expected 2-D diffuser correlations. However, low stagger designs had a much shorter range than predicted. To understand why there is this discrepancy, Koch connected the magnitude of the re-energisation effect to the velocity triangle design by considering the ratio of endwall to freestream inlet dynamic pressure. The black vectors in Figure 2.7 show a typical freestream rotor exit (stator inlet) velocity triangle. The endwall boundary layer is assumed to leave the rotor with the same deviation as the freestream. The axial velocity in the endwall is reduced by $\Delta \mathrm{V}_{\mathrm{x}}$ and the corresponding minimum velocity in the endwall is $\mathrm{V}_{\mathrm{min}}$, shown as the red vector. If either $\alpha_{1}+\beta_{1}$ or $\alpha_{2}+\beta_{2}$, is less than $90^{\circ}, V_{\text {min }}$ must be less than the freestream velocity $V$. 


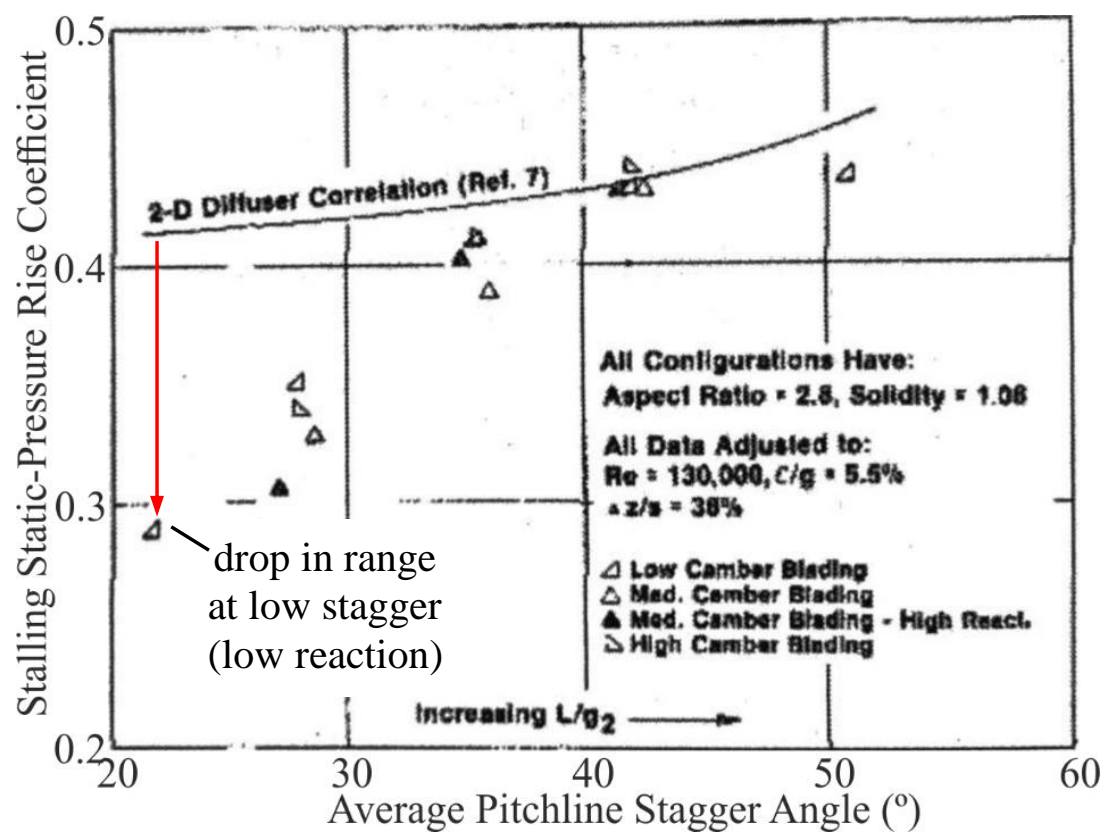

Figure 2.6: Variation of stalling static-pressure rise coefficient with stagger angle, taken from Koch (1981).
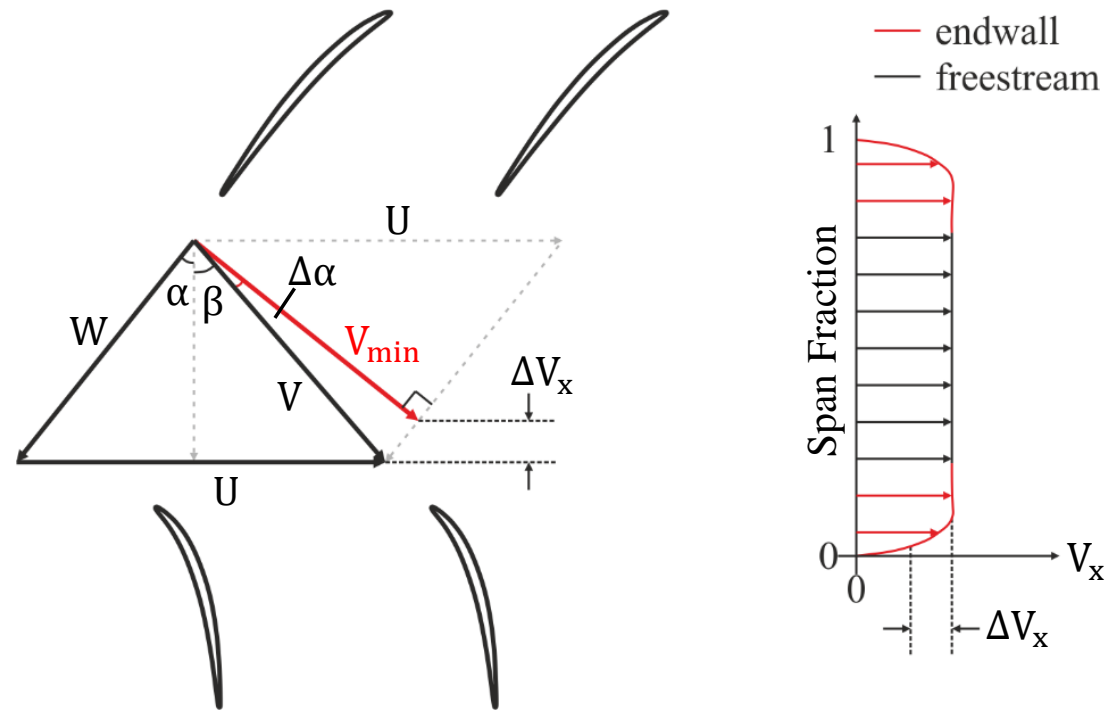

Figure 2.7: Definition of minimum velocity $V_{\min }$ in the 'effective dynamic pressure factor' $F_{e f}$, for an example velocity triangle design. Adapted from Koch (1981).

The ratio $V_{\min }^{2} / V^{2}$ is called the 'effective dynamic pressure factor' $F_{\text {ef }}$ by Koch. This ratio is identical to that given in equation 2.4 when the velocity in the endwall is taken to be $\mathrm{V}_{\mathrm{min}}$. If the actual bladerow inlet dynamic pressure is scaled by $\mathrm{F}_{\mathrm{ef}}$, then the stalling 
static-pressure rise coefficient predicted by the 2-D diffuser correlations, shown in Figure 2.6, shows good agreement with the measured data. The adjusted static-pressure rise coefficient predictions are plotted against $\mathrm{F}_{\mathrm{ef}}$ in Figure 2.8.

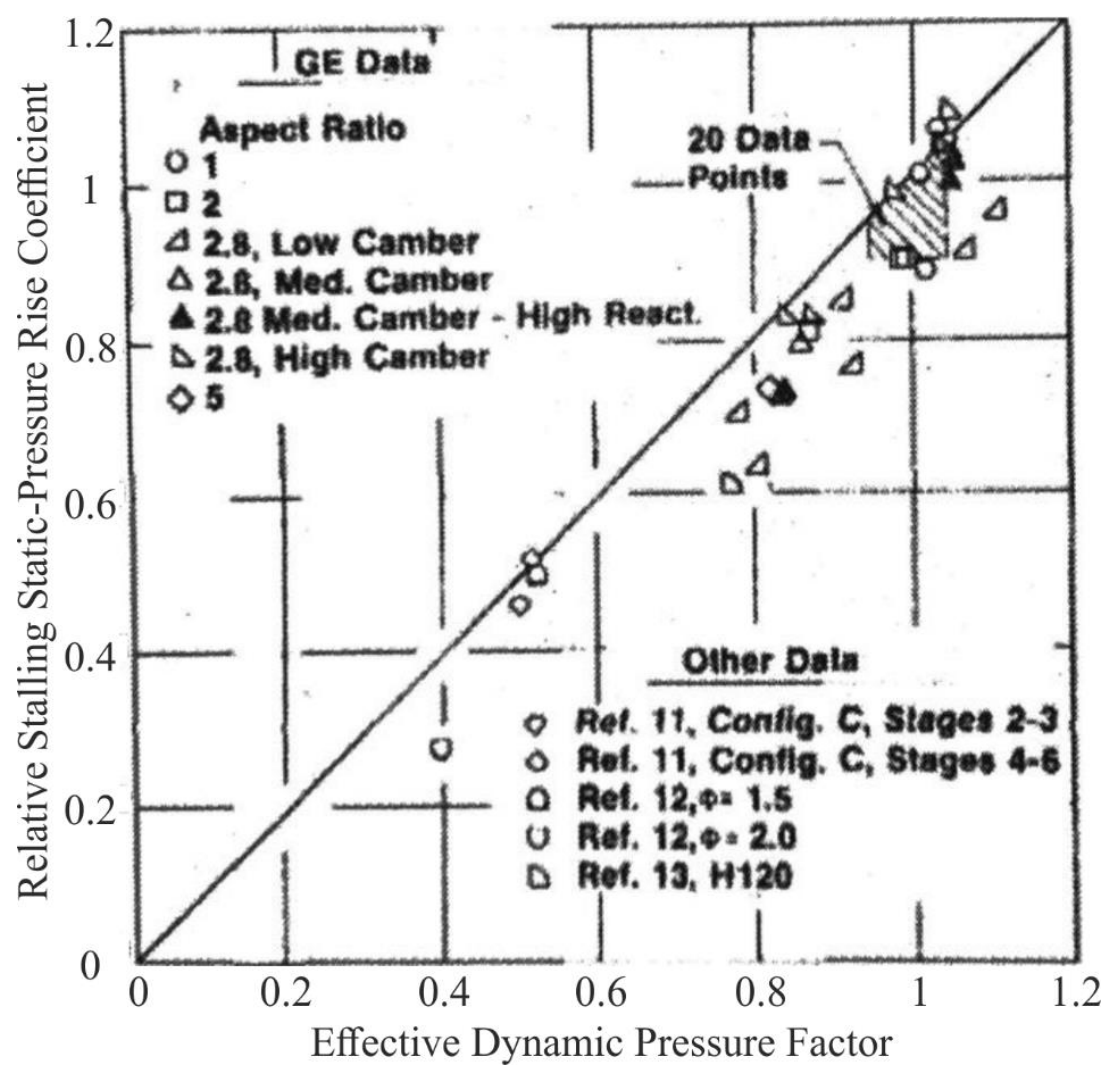

Figure 2.8: Variation of stalling static-pressure rise coefficient with 'effective dynamic pressure factor' $\mathrm{F}_{\mathrm{ef}}$, taken from Koch (1981).

The 'effective dynamic pressure factor' $F_{\text {ef }}$ can be determined from the velocity triangle design alone and is defined by:

$$
F_{e f}=\frac{V_{\min }^{2}}{V^{2}}=\left\{\begin{array}{cl}
\sin ^{2}(\alpha+\beta) & , \text { if }(\alpha+\beta) \leq 90^{\circ} \text { and } \beta>0^{\circ} \\
1 & , \text { if }(\alpha+\beta)>90^{\circ} \\
\frac{U^{2}}{V^{2}} & , \text { if } \beta<0^{\circ}
\end{array}\right.
$$

In a repeating stage, the effect of the change of reference frame on the re-energisation of the endwall boundary layer has a significant effect on the development of the endwall 
boundary layer. This ultimately affects endwall loss and the operating range of the compressor. The ratio of endwall to freestream inlet dynamic pressure was shown by Koch, and later Auchoybur and Miller, to be critical in determining the level of this effect. The 'effective dynamic pressure factor' $\mathrm{F}_{\mathrm{ef}}$ developed by Koch captures the effect, but is limited as is does not capture the effects of varying solidity and the effects of rotation.

\subsubsection{Summary}

Horlock, Cumpsty, Denton and McKenzie employ different arguments, but all come to the same conclusion that $50 \%$ reaction should result in the compressor with the highest efficiency. The problem with these views is that they ignore two realities. First, they do not allow the solidity to vary. Second, they ignore the fundamental difference between the way in which the boundary layers develop in the rotor and stator. LHS shows that the variation in solidity is a critical effect. There are a limited number of studies which look at the effect of systematically varying reaction. However, these are each limited in some way. Studies based on loss correlations do not capture the underlying physics and the experimental results do not have sufficient accuracy. The effect of reaction was captured by the 3-D CFD results of Dickens \& Day and the results showed the asymmetry between the rotor and stator that rotation introduces. However, the study was limited by the difficulty in decoupling the effects of reaction from other effects. Casey investigated how reaction changes the shape of the Smith Chart. His Smith Chart was developed using loss correlations, making it subject to the same problems described above. Finally, the effect of reaction on the re-energisation of the endwall boundary layer has not been studied previously. However, the effect is shown to be significant. Only through 3-D CFD or experiments could the effect of reaction on this effect be studied.

For a study to accurately capture the effect of reaction, what is required is a 3-D CFD study, where the solidity of the stage can be controlled in a systematic way. To include the effect of boundary layer re-energisation in the study, a repeating stage model would also be required. 


\subsection{Effect of rotation on compressor flowfield}

This section is further divided into three parts. The first part studies the effect of rotation on the mid-height boundary layer. The second studies the effect of rotation on corner separations. The third studies the effect of the rotation on boundary layer transition.

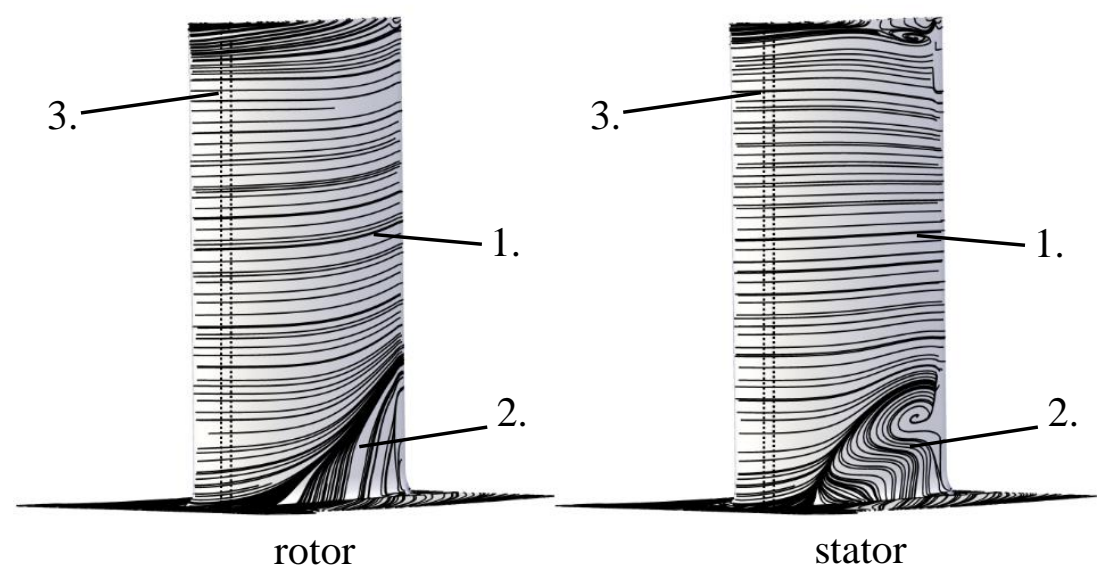

Figure 2.9: Suction-surface limiting streamlines for $50 \%$ reaction, illustrating the asymmetry between loss mechanisms in the rotor and the stator.

\subsubsection{Mid-height boundary layer}

There is a natural asymmetry between the way in which the blade surface boundary layers develop in the rotor and stator. This asymmetry is caused by rotational forces. The effect of the centrifugal and Coriolis forces through the blade surface boundary layers, causes the rotating boundary layers to be accelerated towards the casing.

The earliest studies of the effect of the centrifugal and Coriolis on rotating boundary layers emerged in the early 1950s. These studies were concerned with the threedimensional laminar boundary layer that develops on rotating helical blades. Fogarty (1951) and Mager \& Hansen (1952) were amongst the first to report that integral boundary methods demonstrated that the magnitude of the radial and streamwise components of flow in the boundary layer were comparable. Horlock \& Wordsworth (1965) showed that this radial migration of flow within the boundary layer was dependent 
upon the blade stagger angle and rotational speed. Later studies by Banks \& Gadd (1963), Morris (1981) and Miyake \& Fujita (1974) observed similar results to Horlock \& Wordsworth. All of these early studies rely on simple analytical models of radial boundary layers, which were validated in simple, idealised, rotating disc experiments. For example by Ekman (1905), in his study of the effect of earth's rotation on ocean currents.

To understand why the rotor boundary layers are forced to migrate radially, Horlock \& Wordsworth show that it is necessary to consider the difference between the centripetal force $\rho V_{\theta}^{2} / r$ in the boundary layer and freestream. In the rotor, the circumferential component of the absolute velocity $V_{\theta}$ within the blade surface boundary layer is greater than that in the freestream. This is due to the deficit in relative velocity in the boundary layer $\mathrm{W}_{\mathrm{bl}}$, as illustrated on the right of Figure 2.10, which shows a typical compressor rotor velocity triangle, at a point along the boundary layer streamline shown in red on the left of Figure 2.10. The magnitude of the centripetal force $\rho V_{\theta}^{2} / r$, in the boundary layer is therefore greater than that in the freestream. This produces a component of boundary layer flow which is radially outwards, towards the rotor tip.
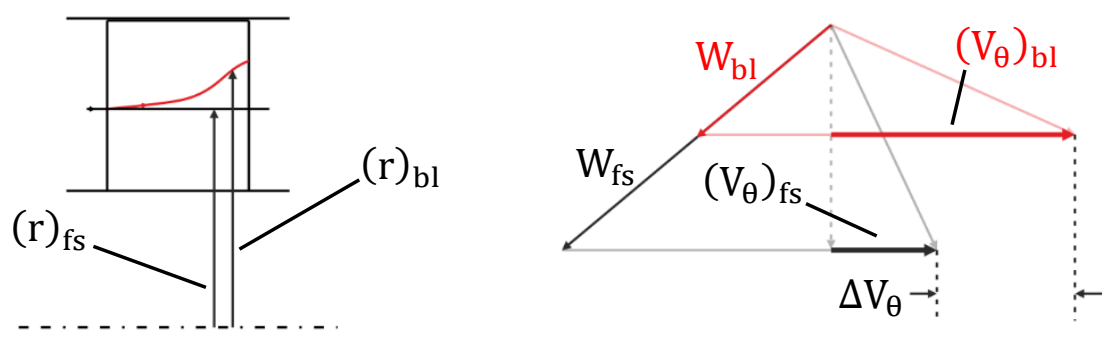

Figure 2.10: LHS: Schematic of blade boundary layer (red) and freestream streamline (black) in the meridional view, in the rotor. RHS: Blade boundary layer (red) and freestream velocity triangle (black).

To understand what controls the extent of the radial flow in the rotor boundary layer, we follow Stratford et al. (1964) and consider the radial equilibrium that must exist between the boundary layer and freestream flow. In a low speed compressor with a plane annulus, the radial flow in the boundary layer is controlled by the radial pressure gradient. To achieve radial equilibrium, the local centripetal force $\rho V_{\theta}^{2} / r$ must balance the local radial 
pressure gradient $\partial \mathrm{p} / \partial \mathrm{r}$ everywhere in the flow, as given by equation 2.6. This local pressure gradient is set up by the bulk passage flow, which in turn, has a circumferential component of absolute velocity $\left(\mathrm{V}_{\theta}\right)_{\mathrm{fs}}$.

$$
\frac{\partial \mathrm{p}}{\partial \mathrm{r}}=\frac{\rho \mathrm{V}_{\theta}^{2}}{\mathrm{r}}
$$

At the same meridional location i.e. the same $\mathrm{x}$-coordinate and r-coordinate, the circumferential component of absolute velocity $\left(\mathrm{V}_{\theta}\right)_{\mathrm{bl}}$ in the boundary layer flow is greater, for the reason described above. This rise in circumferential velocity is denoted by $\Delta \mathrm{V}_{\vartheta}$ in equation 2.7 and is illustrated on the right of Figure 2.10.

$$
\left(\mathrm{V}_{\theta}\right)_{\mathrm{bl}}=\left(\mathrm{V}_{\theta}\right)_{\mathrm{fs}}+\Delta \mathrm{V}_{\theta}
$$

As the boundary layer and freestream flow must be subjected to the same radial pressure gradient, imposed by the bulk passage flow, equation 2.8 follows which equates the centripetal force $\rho V_{\theta}^{2} / r$ in the boundary layer and freestream. For the boundary layer to support the same radial pressure gradient as the bulk passage flow, it must follow a path of radius $(r)_{b l}$, different from that in the bulk flow $(r)_{\mathrm{fs}}$.

$$
\frac{\partial \mathrm{p}}{\partial \mathrm{r}}=\frac{\rho\left(\mathrm{V}_{\theta}^{2}\right)_{\mathrm{fs}}}{(\mathrm{r})_{\mathrm{fs}}}=\frac{\rho\left(\left(\mathrm{V}_{\theta}\right)_{\mathrm{fs}}+\Delta \mathrm{V}_{\theta}\right)^{2}}{(\mathrm{r})_{\mathrm{bl}}}
$$

which ignoring high order terms, re-arranges to:

$$
\frac{(\mathrm{r})_{\mathrm{bl}}}{(\mathrm{r})_{\mathrm{fs}}}=1+2 \frac{\Delta \mathrm{V}_{\theta}}{\left(\mathrm{V}_{\theta}\right)_{\mathrm{fs}}}
$$

In their study of the effect of rotation on the development of turbulent boundary layers, Hughes and Horlock (1971) derive a similar result to that given by equation 2.9. It shows that the extent of the radial migration of the rotor boundary layer, is controlled by the 
ratio of the rise in circumferential velocity between the boundary layer and freestream $\Delta \mathrm{V}_{\theta}$, relative to the freestream circumferential velocity $\left(\mathrm{V}_{\theta}\right)_{\mathrm{fs}}$. In the rotor, $\Delta \mathrm{V}_{\theta}$ and $\left(\mathrm{V}_{\theta}\right)_{\mathrm{fs}}$ must carry the same sign. It therefore follows from equation 2.9 that $(r)_{b l}>(r)_{\mathrm{fs}}$, as shown on the left of Figure 2.10. This means that the rotor surface boundary layer is always accelerated towards the casing.

The high Reynolds number water pump (HIREP) facility described by Zierke et al. (1994a, 1994b) is not of a typical aero-engine design, but does allow detailed study of rotor boundary layer flows using simple oil-paint visualisation techniques. The left of Figure 2.11 shows the surface limiting streamlines on the rotor suction-surface, drawn from the oil-paint visualisation. It shows clearly how the attached rotor boundary layer migrates radially as it approaches the separation line near the rotor trailing-edge. The hub corner separation is observed to be significantly three-dimensional and is also pumped radially outboard. The effect of rotational forces on corner separations is discussed in detail in the next section. The surface shear stress lines on the rotor pressure-surface are shown on the right of Figure 2.11. Compared to the suction-surface, the pressure-surface boundary layer appears to be two-dimensional over most of the blade surface. The radial flow near the blade tip initiates from the pressure-surface and migrates through the rotor tip clearance.

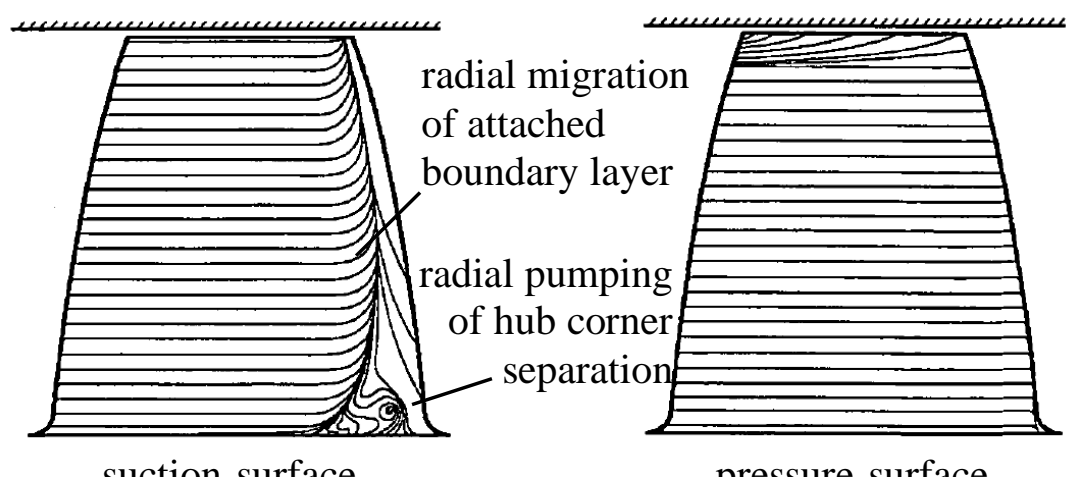

Figure 2.11: Surface limiting streamlines on rotor blade suction-surface (left) and pressure-surface (right), from oil-paint visualisation. Adapted from Zierke (1994a).

The radial flows within the rotor blade boundary layer change the boundary layer profile and growth. Employing integral methods to study laminar boundary layers on rotating 
helical blades, in a similar way to the earlier work of Horlock \& Wordsworth, Lakshminarayana et al. (1972) found that the boundary layer momentum thickness and shape factor both rise with an increase in rotational speed i.e. a rise in the level of rotational forces. Lakshminarayana \& Govindan (1981) and later Karimipanah \& Olsson (1992) observed a similar effect in their studies of the development of turbulent boundary layers in more modern examples of compressor rotor blades. Their calculated values of momentum thickness show good agreement with the rotating hot-wire wake traverse measurements performed by Reynolds \& Lakshminarayana (1979). A rise in boundary layer momentum thickness and shape factor, both with an increase in the level of rotational forces, has been reported numerous times in the literature, however in each case it is not clear why this is observed. Lakshminarayana et al. (1972) also noted that the rotor skin-friction coefficients were $40-60 \%$ higher than those expected in an equivalent stationary flat plate. The measured increase in skin-friction is consistent with earlier work performed by Nash \& Hicks (1971), but contradicts the results observed by Karimipanah \& Olsson (1992) who found the skin friction to vary inversely with rotational speed.

Much of the confusion arising from the results observed above arises from an inability to decouple the effect of changing the velocity triangle and the effect of rotational speed i.e. rotational forces, in a tractable way. A similar confusion arises in three-dimensional design where radial boundary layer flows are induced by both the 3-D design of the blade and the natural transverse pressure gradient. In their three-dimensional Computational Fluid Dynamics (CFD) design study of stators, Taylor \& Miller (2017) were able to decouple the effects of 3-D design from the effects of the transverse pressure gradient, which acts normal to the streamwise direction. The transverse pressure gradient was carefully controlled using an aerodynamically driven, automated design up the span. Outside of the corner separations, the highest suction-surface boundary layer shape factor was observed by Taylor (2016) in the regions of high transverse flow, in a similar way to Lakshminarayana et al. (1972). The rise in shape factor was attributed to the rise in transverse flow. This transverse flow moves low momentum boundary layer flow, from the innermost part of the streamwise component of the boundary layer, to the innermost part. This effect changes the boundary layer profile in such a way to increase the shape factor. Taylor also noted that this effect causes the total design loss to rise. 
It is clear that the one of the key problems in studying the effect of rotation on the mid-height boundary layer is the inability to controllably decouple the radial pressure gradients, caused by rotation, from those caused by blade design. It is therefore important that this study allows for this decoupling.

\subsubsection{Corner separations}

The performance of a compressor, particularly as it approaches its maximum pressure rise as explained by Gbadebo et al. (2005) and Lei et al (2008), depends upon the behaviour of the 3-D endwall corner separations. To understand the effect of rotation on the performance of the compressor, particularly its operating range, it is therefore critical to consider the effect of rotation on corner separations.

A typical corner separation exists in each blade passage where the endwall and suction-surfaces meet, near the trailing-edge. The endwall boundary layer driven across the passage by the cross-passage pressure gradient and accumulates in the suction-surface corner. This causes the formation of a corner separation. The development of corner separations in stationary blade rows is well documented. The development of corner separations in rotating blade rows is not well documented. This is because of the difficulty in undertaking experiments in the rotating frame. The relative velocity in a rotor corner separation is less than that in the bulk passage flow. This means that the rotor corner separation is pumped radially, up the blade span, as described in the previous section. The effect can be seen in the 3-D CFD study of Taylor (2019). Figure 2.12 compares the surface limiting streamlines between the rotor (left) and stator (right) hub corner separations. In the rotor hub corner separation, the region of streamline curvature is not present and the flow does not reverse. This improvement is a direct result of the stronger local radial pressure gradient, forcing the low momentum endwall boundary flow layer up the span. In Taylor's study, it is difficult to decouple the effects of the radial pressure gradient induced by rotation and the effect of the transverse pressure gradient induced by Taylor's 3-D blade design.

The development of corner separations in rotating blade rows is not well understood. This is due in part to the difficulty of using rotating instrumentation. The study of Taylor (2019) is subject to the same problems as described in the previous 
section. In his study it is difficult to decouple decouple the radial pressure gradients, caused by rotation, from those caused by the 3-D blade design. It is critical that this study allows for this decoupling.

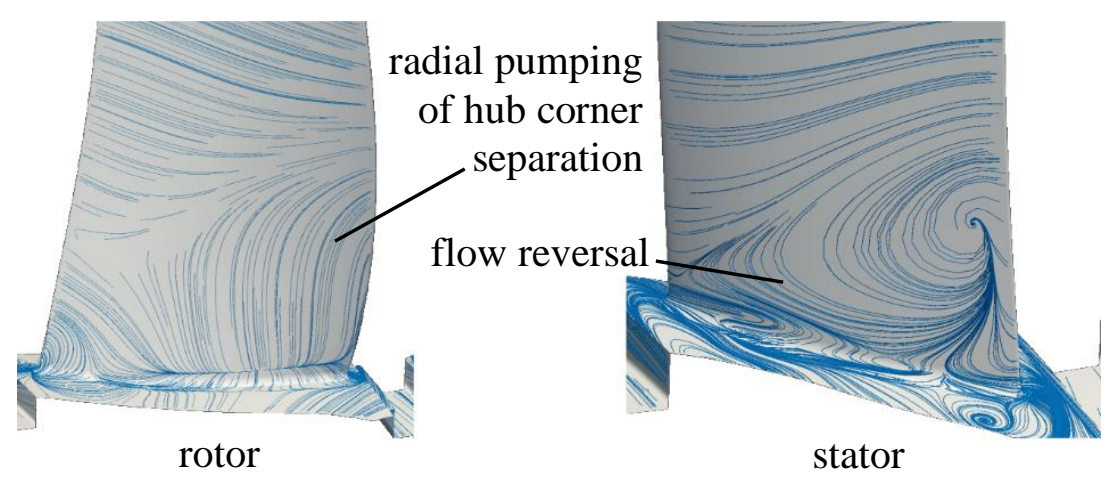

Figure 2.12: Corner separation flow topology of rotor (left) and stator (right). Adapted from Taylor (2019).

\subsubsection{Boundary layer transition}

Little work has been published to date on the effects of rotation on boundary layer transition. The early work of Taylor (1932) recognises that the effect of the centrifugal and Coriolis forces in rotating boundary layers affects the process of transition to turbulence. The notion that rotation promotes early transition due to the effects of the Coriolis force in the boundary layers was examined by Lakshminarayana et al. (1972). They observed that the rotor blade boundary layer transitioned earlier than would be expected in an equivalent stationary flat plate. Karimipanah \& Olsson (1992) demonstrated that the region of transition moved upstream as the level of rotation was increased. This was also observed by Lakshminarayana et al. (1982).

The effect of rotation on the transition mechanism in the development of secondary flow in the turbine was studied by Baum et al. (2016). This shows that rotation acts to promote early transition. Their study assessed, using 3-D CFD, the influence of the Coriolis force on the horseshoe vortex system in a linear turbine cascade. 


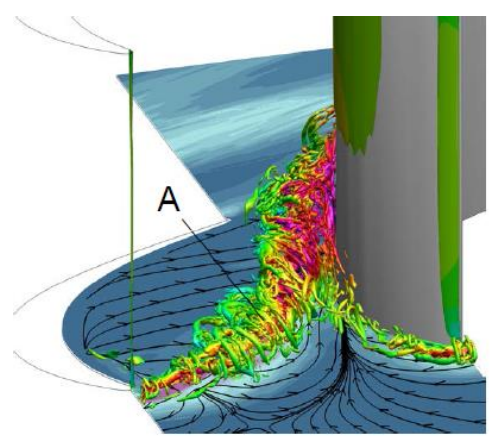

$(\Omega<0)$

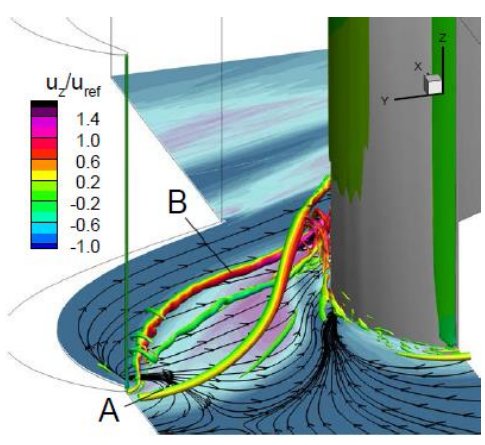

$(\Omega=0)$

Figure 2.13: 2.4 Isosurfaces of $\lambda_{2}$-criterion coloured by spanwise velocity showing the pressure-side leg of the horseshoe vortex with the Coriolis force activated (left) and switched off (right), taken from Baum et al. (2016).

A reference case without Coriolis forces $(\Omega=0)$ and two additional cases with rotation $(\Omega<0)$ and counter-rotation $(\Omega>0)$ were calculated. Their CFD code was modified to include the addition of a Coriolis force term. This term acted everywhere in the computational domain, including in and upstream of the blade passage. This was found to induce an undesirable large-scale secondary flow which displaced the horseshoe vortex system radially. With the Coriolis force activated $(\Omega<0)$, the observed secondary flows were considerably different from the reference case $(\Omega=0)$. The two stable parts of the horseshoe vortex in the reference case, seen in the right of Figure 2.13 and labelled A and $\mathrm{B}$, are not present in the case with rotation as shown in the left of Figure 2.13. Instead a single, much larger, turbulent structure exists, labelled A.

The mechanism by which the Coriolis force influences transition of the horseshoe vortices is illustrated by the schematics shown in in Figure 2.14. Here we follow Baum et al. (2016) and assume that the axis of the vortex is parallel to the axis of rotation. The Coriolis force $\mathrm{f}_{\text {cor }}$ must always act perpendicular to the axis of rotation and the local velocity vector, and therefore in the rotating cases $(\Omega>0)$ and $(\Omega<0)$, the Coriolis force acts radially, as shown in Figure 2.14. For the case where $\Omega<0$ the Coriolis force leads to a contraction of the vortex, increasing the pressure along the axis and stabilising the vortex which promotes transition. This explains why in the left of Figure 2.13, a single turbulent structure exists. 

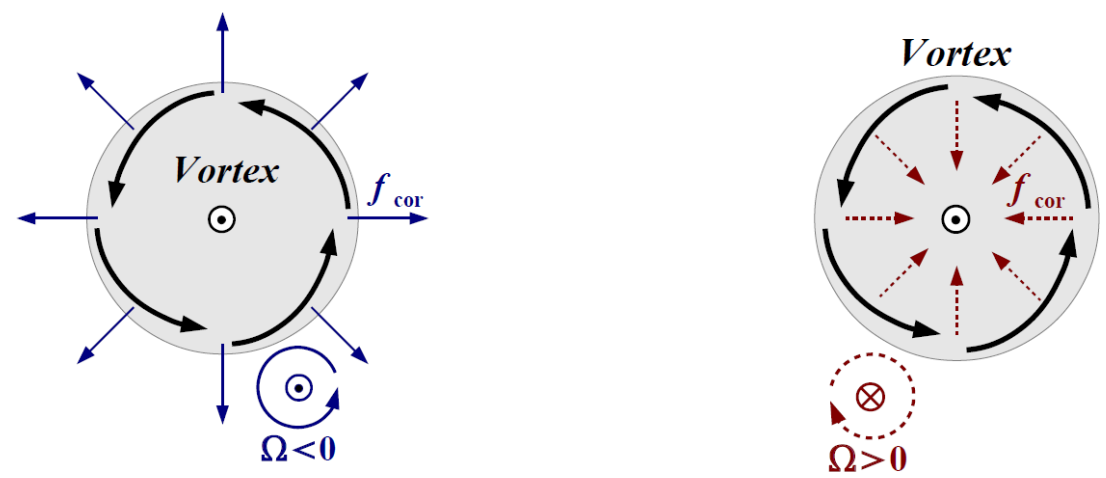

Figure 2.14: Schematic illustrating a vortex aligned with the axis of rotation and the direction of the associated Coriolis force for the case with rotation (left) and opposite rotation (right), taken from Baum et al. (2016).

There have been limited studies on the effects of rotation on boundary layer transition. It was observed in number of experiments that the rotor blade boundary layer transitions earlier than would be expected in an equivalent stator. However in these studies, it is difficult to decouple the effects of rotation from other effects. The 3-D CFD study of Baum et al. (2016) also shows that rotation promotes early transition. However, the way in which the Coriolis force was added to the CFD solution was found to induce an undesirable large-scale secondary flow, which must be avoided in this study.

\subsubsection{Summary}

It should be first noted that rotation has a significant effect on the development of the rotor boundary layer. Towards the trailing-edge of a rotor, rotational forces cause the boundary layer to migrate towards the casing. Second, rotation is also seen to inhibit the formation of the corner separations, by radially pumping the flow. One of the key problems in studying the effect of rotation is the inability to controllably decouple the radial pressure gradients, caused by rotation, from those caused by the blade design. In addition, because of the difficulty of undertaking experiments in the rotating frame, little research has been done to determine the effects of rotation. There is therefore a need to study how rotation changes the flowfield in the rotor in a more controllable way. 


\subsection{Research Questions}

A review of the literature in this chapter has raised a number of questions. These questions are summarised below.

1. Much of the confusion in the field is caused by the inability to be able to decouple the effects of changing reaction on the mid-height velocity triangle and on the effects of rotation on boundary layers. Chapter 3 will describe the development of a computational model which allows these two effects to be decoupled.

2. LHS showed that varying the blade solidity with reaction alters the effect of reaction on profile loss. In Chapter 4 a number of ways of controlling solidity, as reaction is varied, will be studied to examine how reaction affects profile loss.

3. The operating range and design loss of a stage both depend on endwall loss. Little is understood about how endwall loss changes with reaction. An added difficulty is that rotation effects have a significant effect on the endwall flow. In Chapter 5 the effects of rotation will be 'switched off' using the model developed in Chapter 3. The effect of reaction on the endwall flow will then be investigated, without the asymmetric effect of rotation.

4. The operating range and design loss of a rotor both depend on rotation because of the effect it has on the endwall flow. In Chapter 6 the effects of rotation are 'switched on' and the effect on operating range and design loss will be studied.

5. Little understanding exists about how the choice of how reaction varies through a multistage compressor effects its design performance. Chapter 7 will investigate how this choice influences the design efficiency of the compressor. 



\section{Chapter 3}

\section{Computational model}

This chapter describes the setup of the computational model which will be used throughout the thesis. The chapter is divided into five parts. The first part explains the setup of the CFD solver and grid. The second describes the blade design. The third sets out the different methodologies of varying solidity. This is important as the way in which the solidity is chosen is critical to understanding the effect of reaction. The fourth explains the linear repeating stage model. The final part describes the new rotation model which allows the effects of rotation to be switched on and off, without requiring a redesign of the blade row.

\subsection{CFD Setup}

This section is further divided into two parts. The first part explains how the profile loss calculations in this thesis are computed using the program MISES, a coupled Euler boundary layer solver developed by Mark Drela \& Youngren (2008). The second part explains how the endwall loss calculations are computed using the program TBlock, a 3D CFD program developed by John Denton (2015). 


\subsubsection{MISES}

The profile loss calculations presented in this thesis are computed using the program MISES. MISES is a steady 2-D coupled Euler boundary layer solver developed by Mark Drela. The program is well-documented and validated, and has a wide application in both the fields of internal and external aerodynamics, for example by Garzon \& Darmofal (2003) to study the impact of geometric variability on compressor performance. The boundary layers were considered to be fully turbulent. A turbulence intensity of $4 \%$ was used. An example of the passage grid generated by MISES for a 10\% reaction rotor is shown in Figure 3.1. Calculations were performed on a single periodic flow passage. The passage was extended approximately 1.3 chord lengths upstream of the aerofoil LeadingEdge (LE) and approximately 1.2 chord lengths downstream of the aerofoil Trailing-Edge (TE).

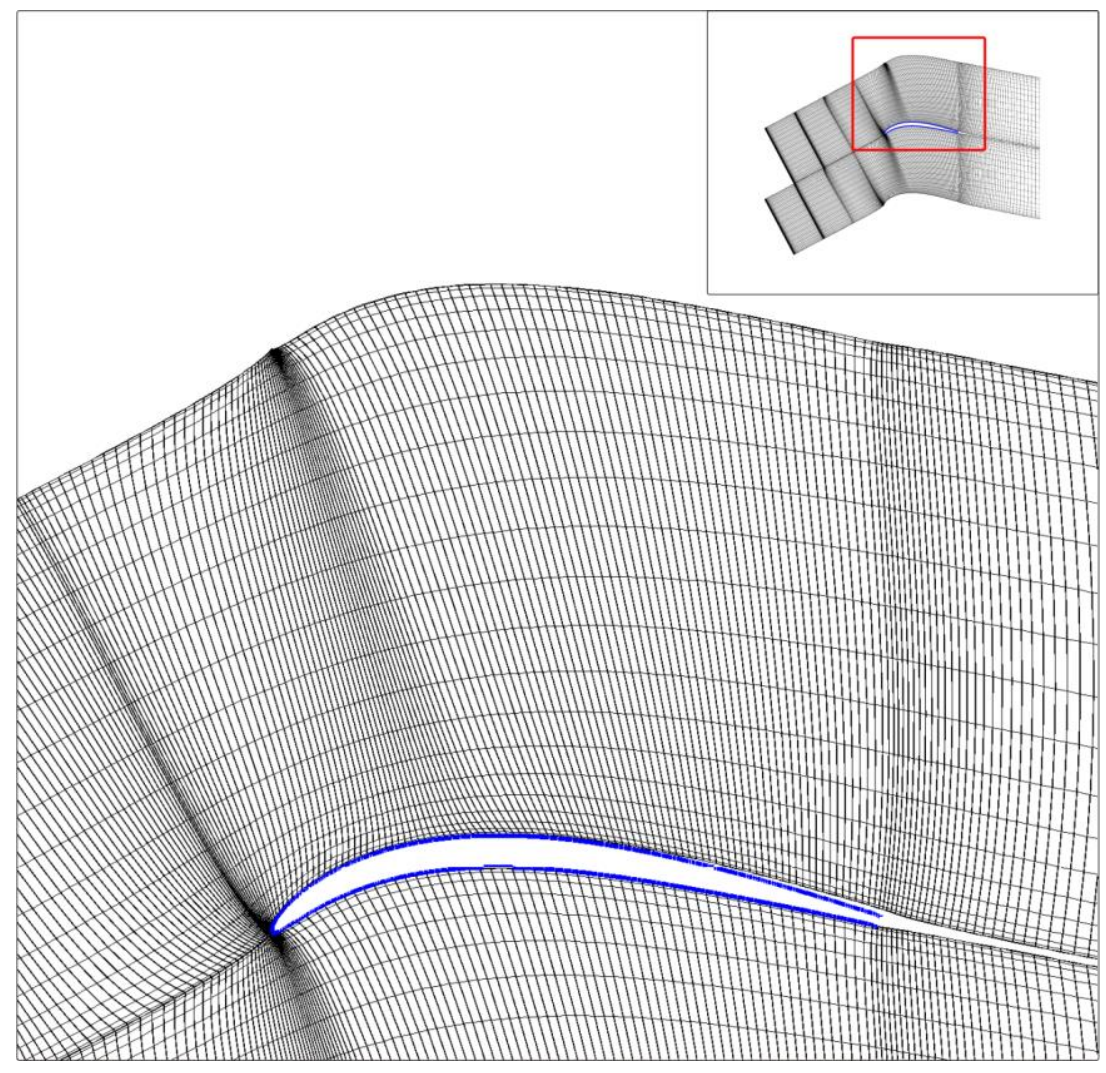

Figure 3.1: Example grid produced by MISES for $10 \%$ reaction rotor. 
The calculations were performed in the same frame of reference of the aerofoil being considered. This means that the rotor aerofoil profile of reaction, $\Lambda$, is identical to the stator aerofoil profile of reaction of $1-\Lambda$. Each aerofoil profile typically took one minute to calculate. MISES was used to design each aerofoil profile. The aerofoil design process is explained in Section 3.2 of this chapter.

\subsubsection{TBlock}

The endwall loss calculations in this thesis are computed using the program TBlock. TBlock is a multi-block structured grid CFD program developed by John Denton (2015). The program is well-documented and validated, and has been used extensively in compressor research and design, for example by Goodhand \& Miller (2011) to study the impact of real geometries on corner separations. TBlock is a fully 3-D, ReynoldsAveraged Navier-Stokes (RANS), finite volume solver. Steady mixing planes were used. The boundary layers were considered to be fully turbulent. The turbulence model used was the one-equation Spalart \& Allmaras (1992) model, with wall functions. The model was adapted by multiplying the turbulent kinetic energy term by a factor of 1.5 . This modification was used in the calculations performed by Taylor (2016), as recommended by the author of the solver to adapt the model for turbomachinery flows. Calculations were performed on a single periodic flow passage. Approximately 1.0 million cells per blade passage were used as a compromise between calculation time and spatial accuracy.

An example of the passage grid generated by TBlock is shown in Figure 3.2 for a $50 \%$ reaction stage. The blade TE was fitted with a TE cusp, as recommended by the author of the solver to minimise artificial entropy generation at the TE due to sudden grid curvature. The length of the cusp was set equal to approximately four times the TE edge radius, as recommended by the author of the solver. H-mesh topology grids were employed to facilitate the calculation of the pitchwise averaged rotation term, described in Section 3.5. A 1.5 stage compressor model (rotor-stator-rotor) was calculated, using a repeating stage model, as described in Section 3.4. The inlet plane was located approximately 0.5 chord lengths upstream of the rotor LE and the exit plane was approximately 1.2 chord lengths downstream of the second rotor TE. 


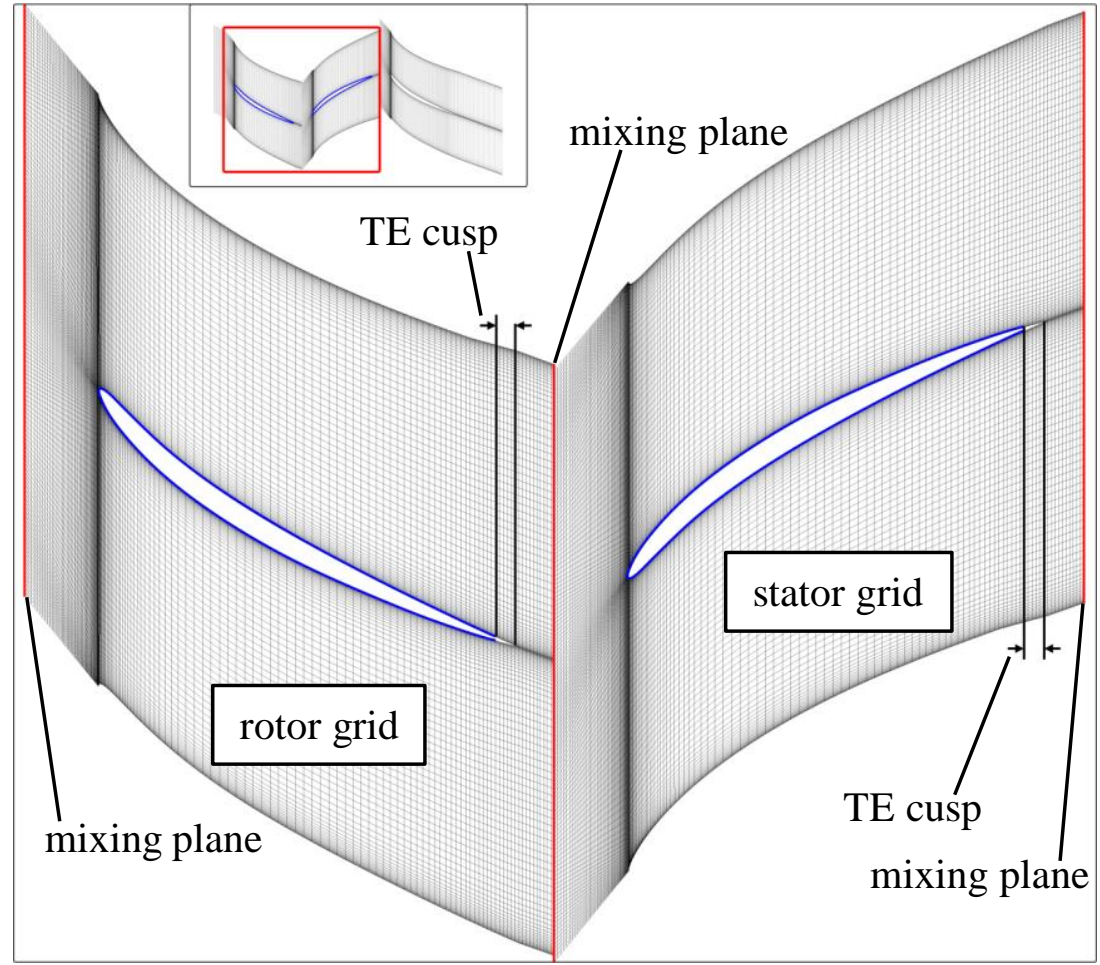

Figure 3.2: Example grid produced by Tblock for $50 \%$ reaction stage.

The viscous boundaries had values of $\mathrm{y}^{+}<1.0$ and $\mathrm{z}^{+}<1.0$ on all solid surfaces. These values are well within the laminar sub-layer of the turbulent boundary layers $\mathrm{y}^{+}<\sim 30$. This was done to increase the accuracy of the wall functions near the viscous boundaries, increasing the accuracy in which the first grid layer velocity gradient is determined. The rotor and stator geometries are two-dimensional, i.e. no sweep or lean was applied. An example of the first grid layer generated by TBlock is shown in Figure 3.3 for a $50 \%$ reaction stage.

The compressor designs were cantilevered with a plane annulus, as shown schematically in the top of Figure 3.4. The aspect ratio was set equal to 2, as done by To (2016) and Auchoybur (2017). The rotor tip and stator hub clearances were equal and set to $1 \%$ of the chord length. Endwall fillets were modelled at the blade-endwall interface. The fillet surface was determined using a 'rolling ball' calculation, described by Klass \& Kuhn (1992). The fillet radius was constant and equal to approximately $5 \%$ of the chord length. 


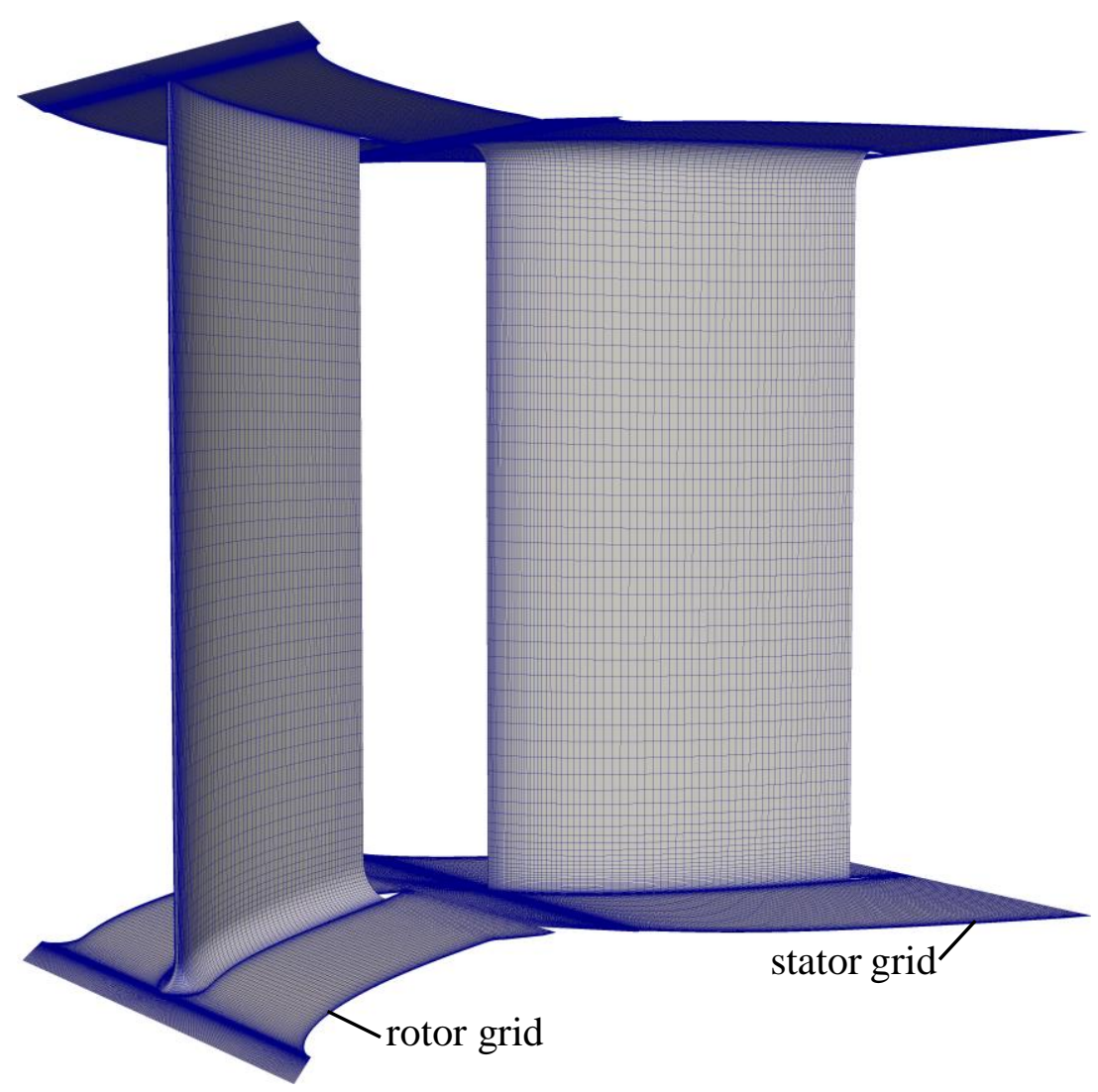

Figure 3.3: Example first grid layer produced by Tblock for $50 \%$ reaction stage.

For the cases with clearances, a fully modelled rotor tip clearance and stator hub clearance, equal to $1 \%$ of the chord length, were gridded with approximately 32,000 cells. In this study only cantilevered rotors and stators were used. Shrouded blade rows were not considered. The hub and casing endwalls were designed to be identical. This means that at $50 \%$ reaction, the hub and casing endwalls reach the same maximum staticpressure rise coefficient. In a real compressor cantilevered rotors and shrouded stators are often used. This means that at 50\% reaction, one endwall reaches its maximum staticpressure rise capability before the other, introducing an asymmetry. In this controlled study, this geometric asymmetry is undesirable and so it was decided that identical hub and casing endwalls would be modelled.

As discussed in Section 2.1.1, as the reaction is raised, the rotor stagger increases. In this study the true-chord $\mathrm{c}$ of the rotor is kept constant, hence as the reaction rises, the rotor axial-chord $c_{\mathrm{ax}}$ decreases. The opposite occurs in the stator. This means that there is a choice to be made about how the axial-gap, $\mathrm{g}_{\mathrm{ax}}$, between the blade rows should vary 
with reaction. It was decided that at any value of reaction, the axial-gap between the blade rows should scale with the axial-chord of the upstream blade row. This ensures that the length over which mixing occurs downstream of a blade row, $\mathrm{g}_{\mathrm{w}}$, is independent of reaction. This can be understood by considering the schematic in the bottom of Figure 3.4. The axial gap between blade rows was set equal to $40 \%$ of the axial-chord length of the upstream blade row. The only exception to this was that the axial distance between the TE of the second rotor and the exit plane. This distance was set to approximately 3 axial-chord lengths to help stabilise the model. The location of the mixing planes was always chosen to be coincident with the centre of the inter-blade row gap.
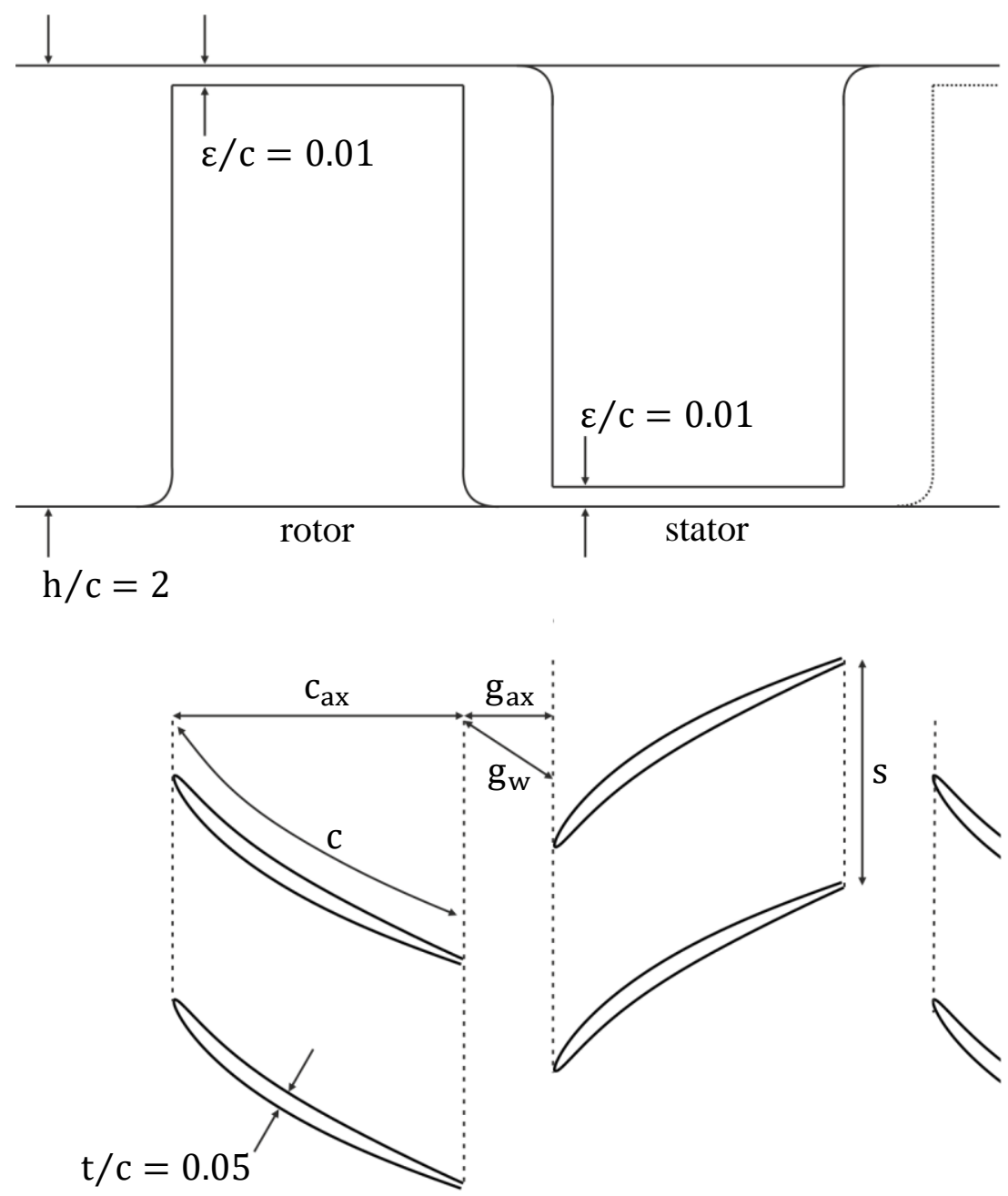

Figure 3.4: Compressor design in meridional (top) and blade-to-blade view (bottom). 
Calculations were performed on the Darwin supercomputer of the University of Cambridge High Performance Computing (HPC) service. Each calculation was performed using the linear repeating stage model described in Section 3.4. Each calculation typically took 18 hours to calculate. To compute a single compressor characteristic, the approximate number of converged calculations performed was 24 .

\subsection{Aerofoil profile design}

This section is further divided into three parts. The first part explains the aerofoil profile parameterisation which was used to define aerofoils profiles. This parameterisation was developed by To (2016). The second part explains how the aerofoil profiles were designed to satisfy the aerodynamic design parameters which define the stage. The third part explains how this is achieved using an iterative procedure, implemented using MISES.

\subsubsection{Aerofoil profile parameterisation}

The aerofoil profile parameterisation used to generate the aerofoil profiles in this thesis follows that of To (2016). The essence of this parameterisation is described in this section and a detailed description is given in Chapter 3 of To (2016).

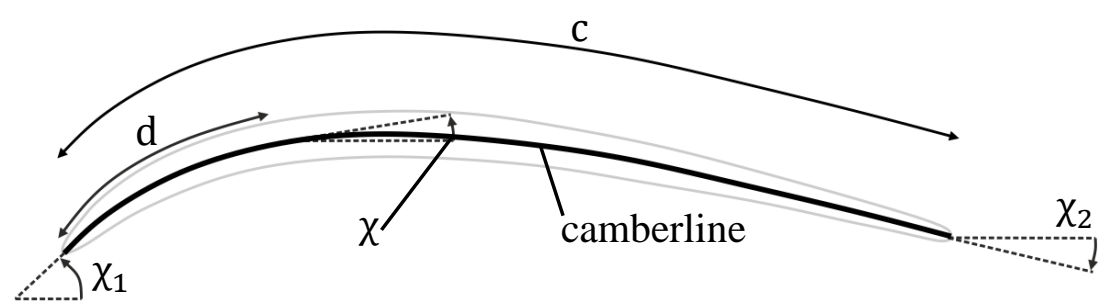

Figure 3.5: Definition of camberline and blade metal angle, $\chi$.

The aerofoil profile is defined by imposing a thickness distribution onto a camberline, as is common practise in preliminary design. The camberline is defined by the nondimensional blade metal angle distribution, $\bar{\chi}\left(\frac{d}{c}\right)$, and the camberline shape is fixed using 
two control points and a cubic polynomial. Determination of the location of these two control points is discussed in Section 3.2.3. An example of a camberline is shown in Figure 3.5 for a $10 \%$ reaction rotor. The blade metal angle, $\chi\left(\frac{d}{c}\right)$, is defined along the camberline, $\left(\frac{d}{c}\right)$, where $\mathrm{c}$ is the true-chord, defined as the total curvilinear distance along the camberline and $\mathrm{d}$ is the curvilinear distance along the camberline from the LE. The non-dimensional blade metal angle distribution is given by equation 3.1:

$$
\bar{\chi}\left(\frac{d}{c}\right)=\frac{\chi\left(\frac{d}{c}\right)-\chi_{1}}{\chi_{2}-\chi_{1}}
$$

where $\chi_{1}$ and $\chi_{2}$ are the inlet and exit metal angles. An example of a non-dimensional blade metal angle distribution is shown in Figure 3.6 for a $10 \%$ reaction rotor. The control points are located at $20 \%$ and $80 \%$ distance along the camberline. Figure 3.7 shows the corresponding non-dimensional thickness distribution, which is imposed onto this camberline. The dashed line represents the line of constant maximum thickness set equal to $5 \%$ of the chord length. At the TE, the thickness is not equal to zero but instead the aerofoil profile is left 'open'. This is because in MISES a blunt TE is specified by leaving the blade 'open' and the solver uses a blunt trailing edge model to account for the associated additional losses, Drela \& Youngren (2008). Near the LE, the gradient of the non-dimensional thickness distribution approaches infinity, which makes it difficult to parameterise. 


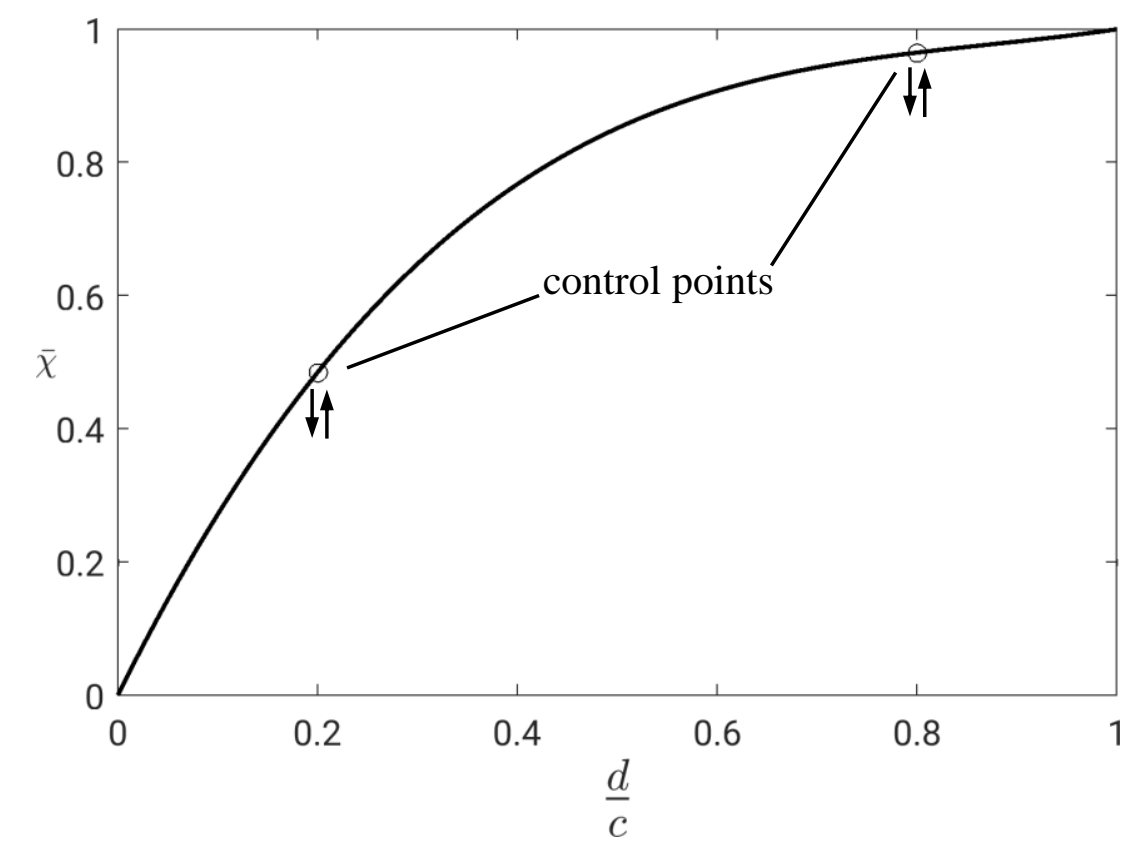

Figure 3.6: Non-dimensional blade metal angle distribution for $10 \%$ reaction rotor.

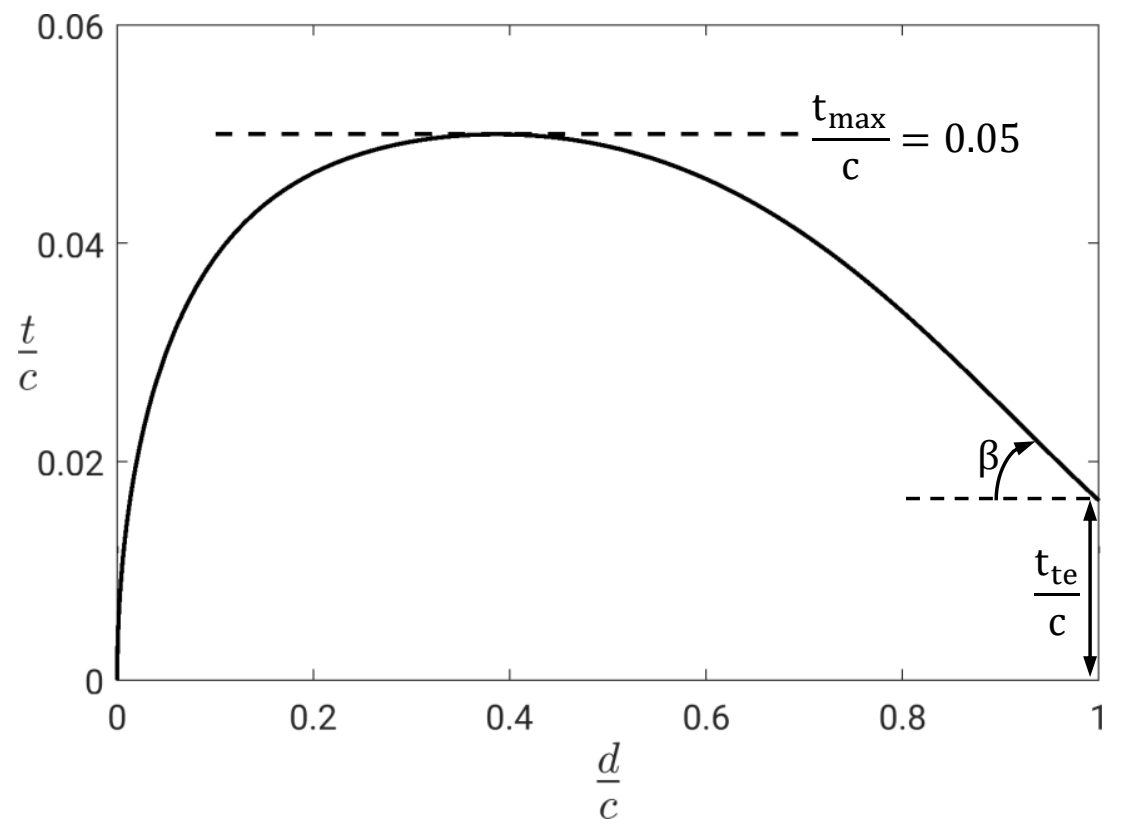

Figure 3.7: Non-dimensional thickness distribution.

To overcome this problem, To (2016) employs a mathematical transformation defined by Kulfan (2008), which transforms the gradient at the LE to a finite value. The transformation is given by equation 3.2: 


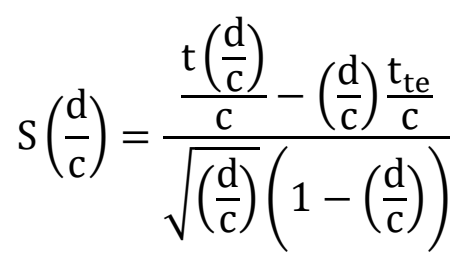

where, $S\left(\frac{d}{c}\right)$ is the transformed non-dimensional thickness distribution and $t\left(\frac{d}{c}\right)$ is the original non-dimensional thickness distribution. Performing this transformation on the non-dimensional thickness distribution shown in Figure 3.7 gives the transformed nondimensional thickness distribution shown in Figure 3.8. The dashed line represents the transformed line of constant maximum thickness. Where the line of constant maximum thickness meets the transformed non-dimensional thickness distribution at a tangent represents the point of maximum thickness.

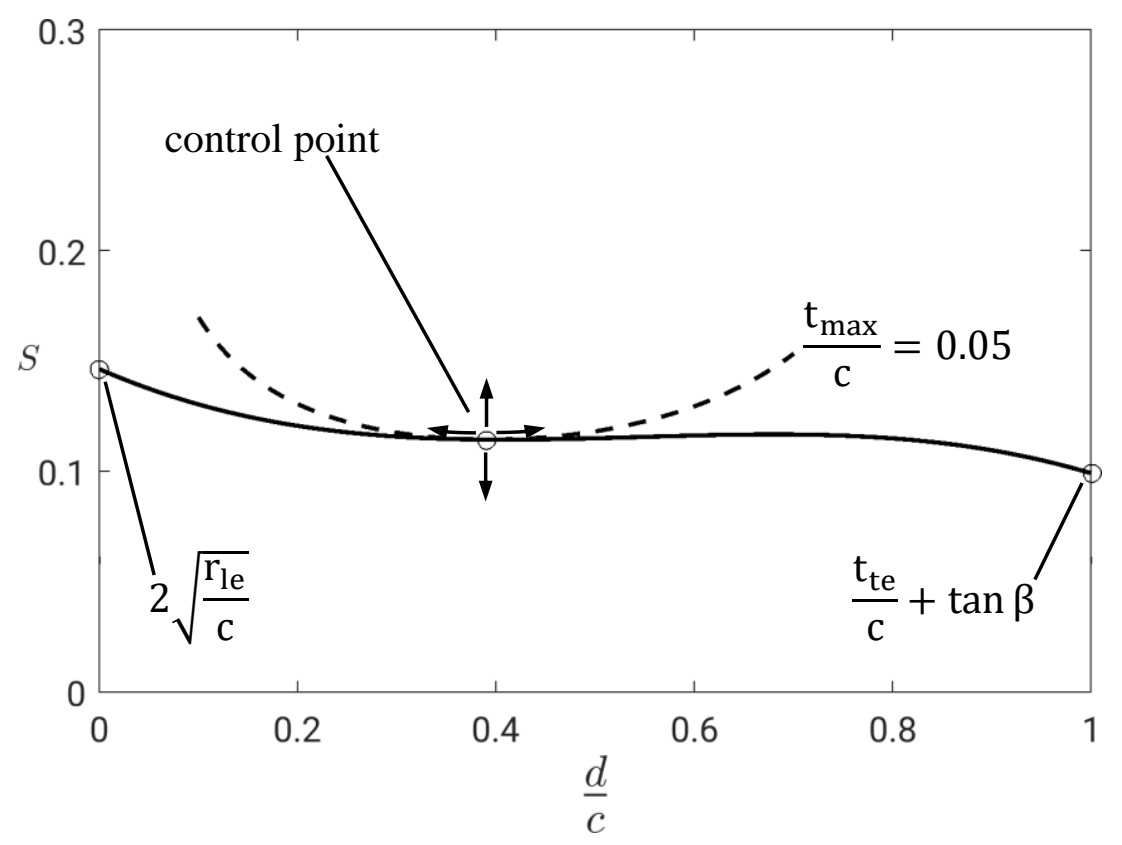

Figure 3.8: Non-dimensional transformed thickness distribution.

The shape of the transformed thickness distribution is fixed using a single control point, two fixed points and a cubic polynomial. The properties of the transformation mean that these three points can be interpreted in a physical way. The fixed point corresponding to 
$S(0)$ sets the LE radius and is given by equation 3.3. The fixed point corresponding to $S(1)$ sets the TE thickness for a given TE wedge angle, $\beta$, as is given by equation 3.4. The control point coincident with the position of maximum thickness controls the maximum thickness and the location of maximum thickness.

$$
\begin{gathered}
S(0)=2 \sqrt{\frac{r_{\mathrm{le}}}{\mathrm{c}}} \\
\mathrm{S}(1)=\tan \beta+\frac{\mathrm{t}_{\mathrm{te}}}{\mathrm{c}}
\end{gathered}
$$

\subsubsection{Aerodynamic design}

The blade parameterisation developed by To (2016) and described in Section 3.2.1 was used to define aerofoil profiles, designed to satisfy the aerodynamic design parameters which define the stage. The performance of a compressor stage is a function of many nondimensional design parameters. In this thesis, the number of parameters is reduced for simplicity so that the effect of reaction and rotation can be studied in isolation. The design is limited to the typical design choices available to a compressor designer trying to design the central stages of a multistage compressor. Equation 3.5 describes the typical choices available:

$$
(P R, \eta)=f\left(\Phi_{d}, \Psi_{d}, \Lambda_{d}, M_{u}, \sigma, A R, t / c, \varepsilon / c, R_{c}\right)
$$

In this thesis a design flow coefficient $\Phi_{d}$ of 0.597 and work coefficient $\Psi_{d}$ of 0.436 is chosen, these values are the same as used by To \& Miller (2019). The blade speed Mach number $\mathrm{M}_{\mathrm{u}}$ is set to 0.3 . The aspect ratio $A R$ is set as 2.0. The values of maximum thickness-to-chord, t/c, and rotor tip clearance-to-chord, $\varepsilon / c$, are fixed as 0.05 and 0.01 respectively. In this study, the size of the rotor tip gap is constant along the chord and equal to the size of stator hub gap. The stage length is constant, which fixes the rotor and stator Reynolds numbers $\operatorname{Re}_{c}$. At $50 \%$ reaction the rotor and stator $\operatorname{Re}_{c}$ are equal to $10^{6}$. The remaining design choices are described by equation 3.6: 


$$
(P R, \eta)=f\left(\Lambda_{d}, \sigma\right)
$$

where $\Lambda_{\mathrm{d}}$ is the design reaction and $\sigma$ is the solidity of the stage. The design point of the aerofoil profiles used in this thesis was set at a design flow coefficient $\Phi_{\mathrm{d}}$ of 0.597 and work coefficient $\Psi_{\mathrm{d}}$ of 0.436 is chosen, except for in Section 4.3 where different locations on the Smith Chart are considered.

\subsubsection{Aerofoil design algorithm}

The aerofoil profiles in this thesis are of a controlled diffusion aerofoil type, designed using MISES to ensure that a given aerodynamic design is achieved. The aerofoil profiles are designed to achieve a specified velocity triangle design i.e. set of flow coefficient, $\Phi_{\mathrm{d}}$, work coefficient, $\Psi_{\mathrm{d}}$, and reaction, $\Lambda_{\mathrm{d}}$. The aerofoil profiles are designed so that the stagnation streamline always bifurcates on the nose of the aerofoil. This condition was originally referred to by Smith (1970), and then later by To (2016) and Auchoybur (2017), as the 'smooth flow' condition. The aerofoil profiles are designed with a 'linear shape factor philosophy'. The camberline distribution is adjusted so that the suction-surface shape factor increases linearly from the peak suction point to the trailing edge, as a function of the distance along the suction-surface.

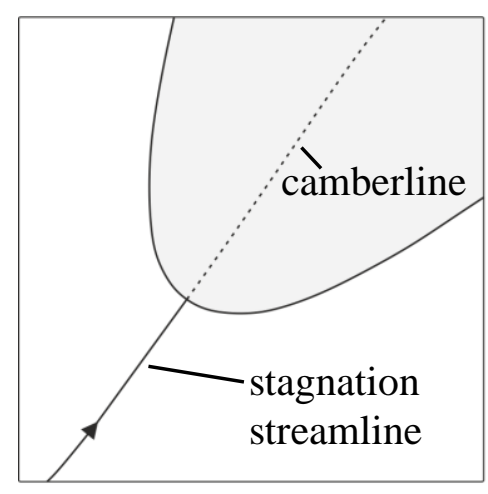

'smooth flow'

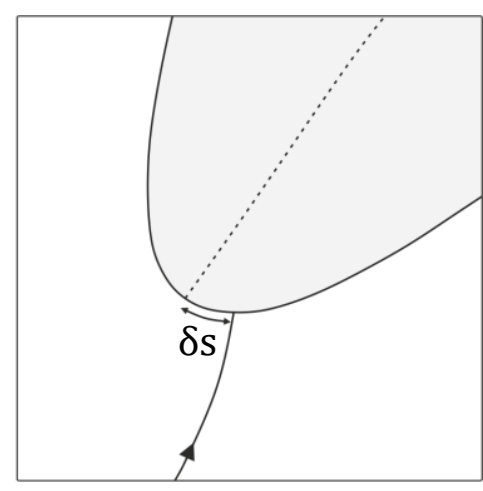

local incidence

Figure 3.9: Definition of 'smooth flow' condition (left) and local incidence (right).

To automate the process of generating aerofoil profiles, a program was written in MATLAB. The algorithm developed determines the required aerofoil inlet and exit metal 
angles and camberline distribution. To calculate the aerofoil inlet and exit metal angles, an iterative process was developed as illistrated in the flow chart in Figure 3.10. This part of the implemented program is based on the Blade Profile Generator program developed by To (2016). An initial guess for $\chi_{1}$ and $\chi_{2}$ are used to generate the initial aerofoil profile. This initial aerofoil profile is then calculated in MISES to determine the surface arc length, $\delta s$, between the stagnation point and the nose of the aerofoil, illustrated on the right of Figure 3.9. If this length is above a tolerance (set as $0.01 \%$ of chord length), then $\chi_{1}$ is readjusted to generate a new aerofoil profile which is re-calculated in MISES. This loop, shown on the left of Figure 3.10, for adjusting $\chi_{1}$ is repeated until the required tolerance has been met so that the 'smooth flow' condition is achieved, as shown on the left of Figure 3.9. Once the 'smooth flow' condition has been achieved, the exit flow angle from MISES is compared with the required exit flow angle. If this is not within a required tolerance (set at $0.1^{\circ}$ ), then $\chi_{2}$ is readjusted and the aerofoil is once again recalculated in MISES. This loop, shown on the right of Figure 3.10 for adjusting $\chi_{2}$, is repeated until the required exit flow angle is achieved to within the required tolerance. Once the required exit flow angle has been achieved, the 'smooth flow' condition is checked again. If the 'smooth flow' condition is no longer satisfied after readjustment of $\chi_{2}$, the entire process is repeated.

To calculate the desired camberline distribution, an iterative process was developed as shown in the flow chart Figure 3.11. This part of the implemented program is based on the technique developed by Taylor (2016). Once $\chi_{1}$ and $\chi_{2}$ have been achieved to within the required tolerance, the suction-surface boundary layer shape factor distribution is computed from MISES. This distribution is compared to the desired case where the suction-surface boundary layer shape factor increases linearly from the peak suction point to the trailing-edge, shown as the red line in Figure 3.12. Two parameters are then calculated corresponding to the two areas between the calculated shape factor distribution and the desired, shown as the black line in Figure 3.12. These two areas are shown as $A_{1}$ and $A_{2}$ in Figure 3.12. 


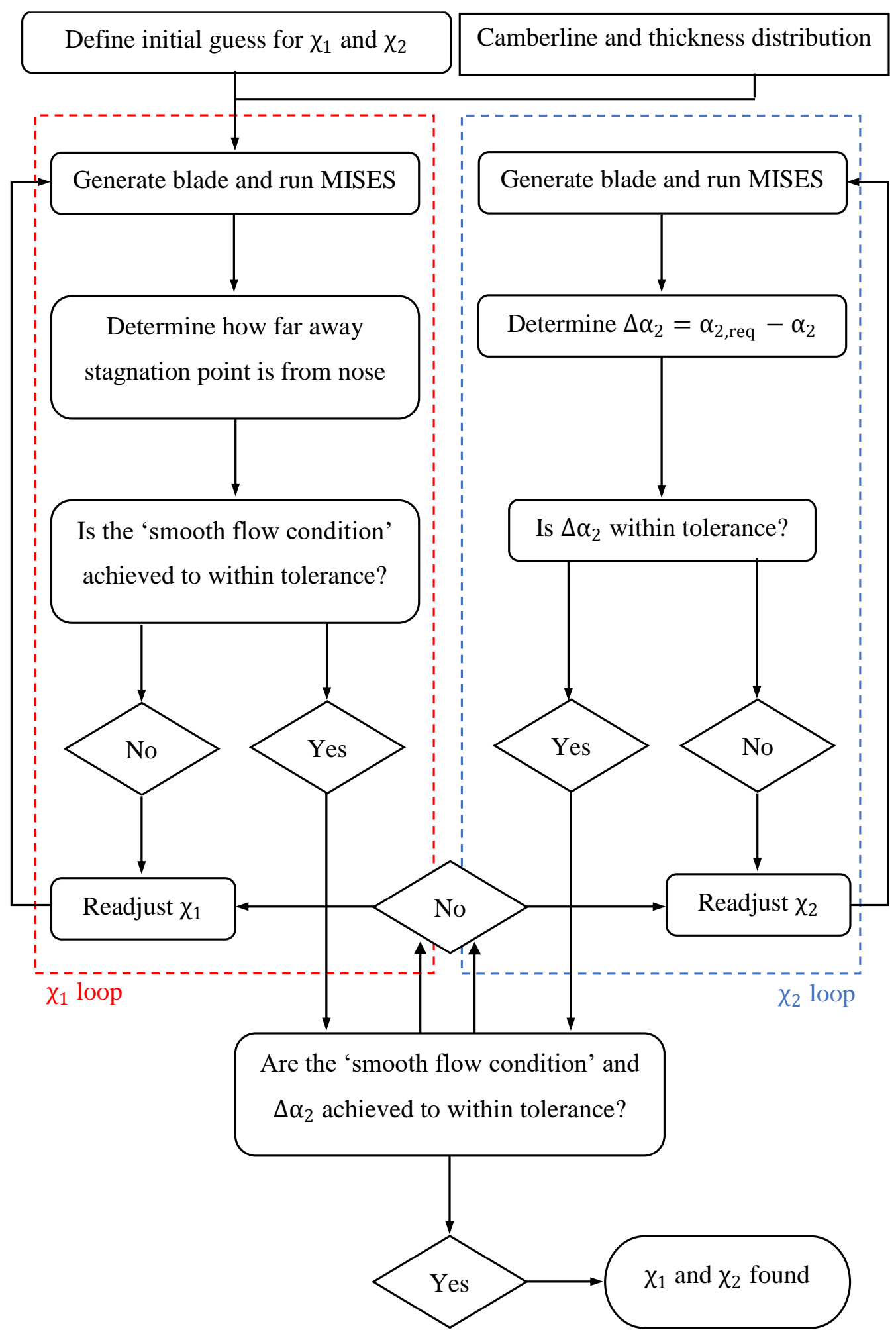

Figure 3.10: Flow chart of setting blade inlet and exit metal angles. 
To drive the calculated shape factor distribution towards the desired distribution, the control points in Figure 3.6, which control the shape of the camberline, are readjusted to produce a new aerofoil which minimises $A_{1}$ and $A_{2}$. The new aerofoil profile is then recalculated in MISES. This loop, shown in Figure 3.11, for adjusting the camberline is repeated until the required tolerances have been met and an aerofoil profile is successfully produced.

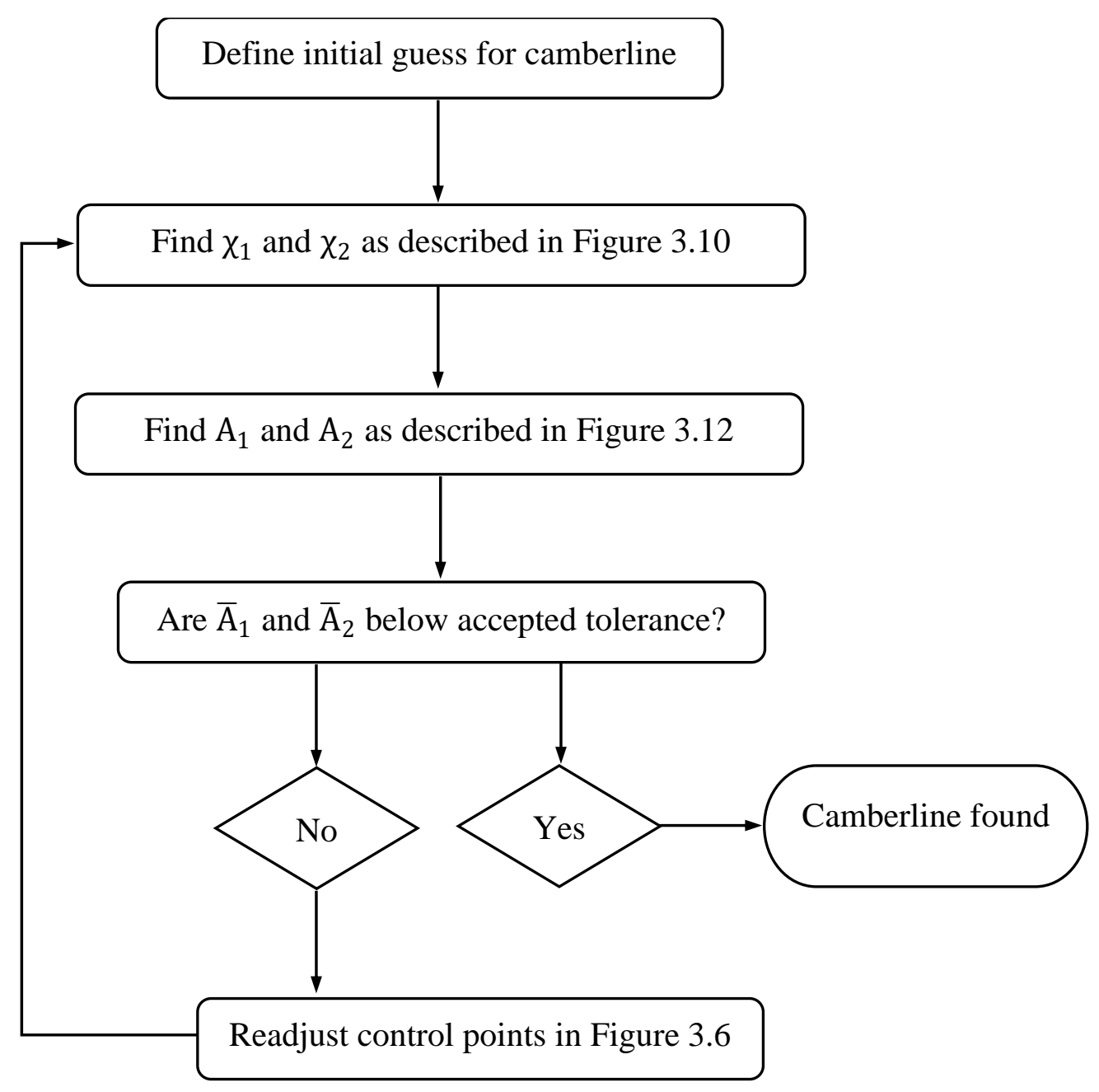

Figure 3.11: Flow chart of setting camberline distribution. 


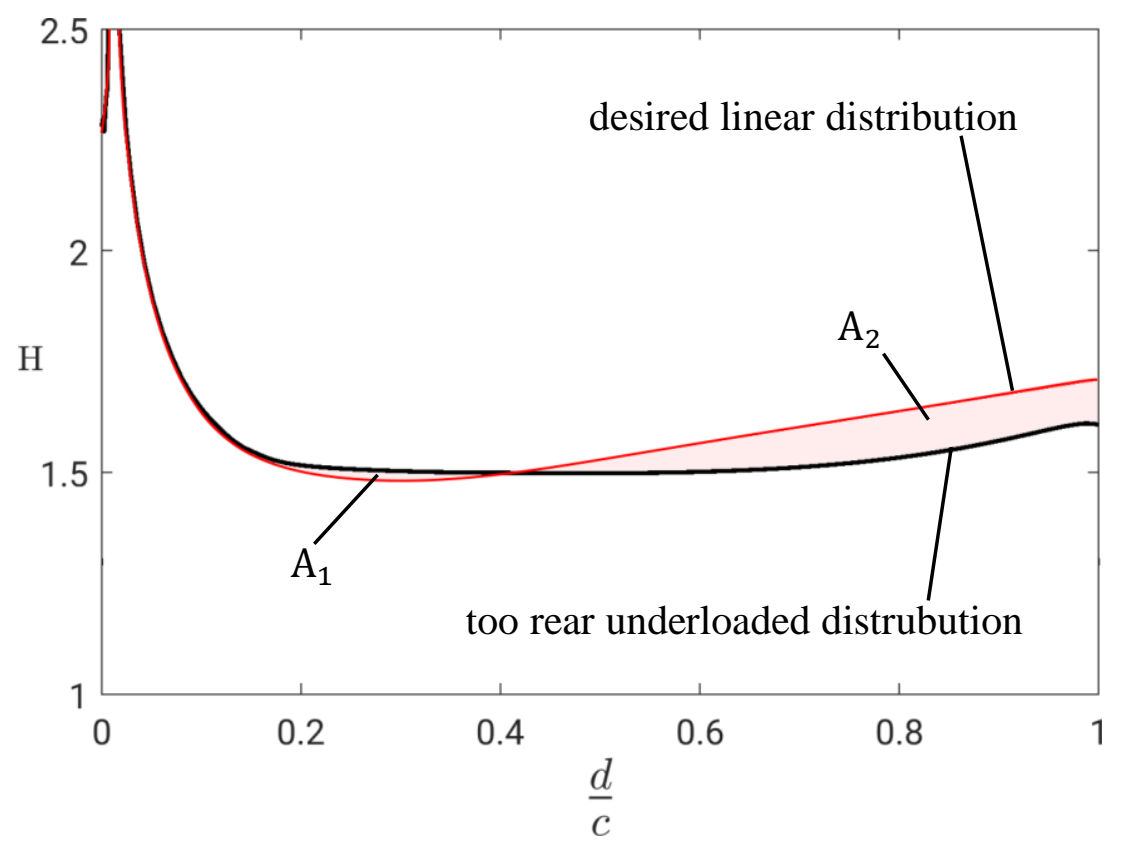

Figure 3.12: Definition of linear shape factor (MISES).

\subsection{Setting solidity}

This section is further divided into three parts. First the solidity is set constant. Second the solidity is set by fixing the equivalent diffusion ratio $\mathrm{D}_{\mathrm{eq}}{ }^{*}$. Third the solidity is set by fixing shape factor of the suction-surface boundary layer at the blade trailing-edge $\mathrm{H}_{\text {te }}$.

\subsubsection{Constant solidity}

For the case of constant solidity, the solidity was chosen to be equal at all reactions to the value at $50 \%$ reaction. The level of solidity at $50 \%$ reaction was chosen to achieve a Diffusion Factor DF equal to 0.45. This method represents the case where the rotor and stator blade numbers are equal, across all reactions. Here we consider the DF equation in its simplest form, given by equation 3.7 :

$$
\mathrm{DF}=1-\mathrm{DH}+\frac{\Delta \mathrm{V}_{\theta}}{2 \mathrm{~V}_{\mathrm{in}} \sigma}
$$


where DH is the blade row de Haller number. Equation 3.7 rearranges to:

$$
\sigma=\frac{\Delta \mathrm{V}_{\theta} / 2 \mathrm{~V}_{\text {in }}}{\mathrm{DF}-(1-\mathrm{DH})}
$$

For a flow coefficient $\Phi_{d}$ of 0.597 , work coefficient $\Psi_{d}$ of 0.436 , reaction $\Lambda_{d}$ of $50 \%$ and DF of 0.45 , the corresponding solidity given by equation 3.8 is approximately 1.49 .

\subsubsection{Solidity set by equivalent diffusion ratio}

For the case of constant Equivalent Diffusion Ratio $D_{\text {eq }}^{*}$, the solidity is chosen so that the level of solidity in the rotor and stator, at each reaction, achieves $\mathrm{D}_{\mathrm{eq}}^{*}$ equal to 1.78 . This is the method adopted by LHS, so that he could use the correlations outlined by Leiblein. This method represents the case where the rotor and stator blade numbers are chosen so that the level of diffusion across the rotor and stator are equal, across all reactions. Here we consider the $\mathrm{D}_{\text {eq }}^{*}$ equation as given by equation 4 in Leiblein (1959):

$$
\mathrm{D}_{\mathrm{eq}}{ }^{*}=\frac{\cos \left(\beta_{2}\right)}{\cos \left(\beta_{1}\right)}\left[1.12+0.61 \frac{\cos ^{2}\left(\beta_{1}\right)}{\sigma}\left(\tan \left(\beta_{1}\right)-\tan \left(\beta_{2}\right)\right)\right]
$$

which can be re-written as:

$$
\mathrm{D}_{\mathrm{eq}}{ }^{*}=\frac{1}{\mathrm{DH}}\left[1.12+0.61 \cos \left(\beta_{\mathrm{in}}\right) \frac{\Delta \mathrm{V}_{\theta}}{\mathrm{V}_{\mathrm{in}, \mathrm{ref}} \sigma}\right]
$$

which re-arranges to:

$$
\sigma=\frac{0.61 \cos \left(\beta_{\text {in }}\right) \Delta \mathrm{V}_{\theta} / \mathrm{V}_{\mathrm{in}}}{\mathrm{D}_{\mathrm{eq}}{ }^{*} \mathrm{DH}-1.12}
$$

where $\beta_{\text {in }}$ is the relative inlet angle to the blade row. For a flow coefficient $\Phi_{\mathrm{d}}$ of 0.597 , work coefficient $\Psi_{\mathrm{d}}$ of 0.436 , reaction $\Lambda_{\mathrm{d}}$ of $50 \%$ and $\mathrm{D}_{\mathrm{eq}}^{*}$ of 1.78 , the corresponding 
solidity given by equation 3.11 is approximately 1.49 i.e. at $50 \%$ reaction, $\mathrm{D}_{\text {eq }}^{*}=1.78$ corresponds to DF $=0.45$.

\subsubsection{Solidity set by trailing-edge shape factor}

For the case of constant trailing-edge shape factor, the solidity is chosen by setting the mean upper limit of the suction-surface boundary layer shape factor at the trailing-edge $\mathrm{H}_{\text {te }}$ equal to 1.71 . This method is common practise in preliminary design and represents the case where the rotor and stator blade numbers are chosen so that the level of the adverse pressure gradient across the rotor and stator boundary layers is equal, across all reactions. This method of setting solidity uses MISES and requires an additional iterative process, to calculate the solidity. The additional iterative process is shown in Figure 3.13 and is executed using the process described in Figure 3.10.

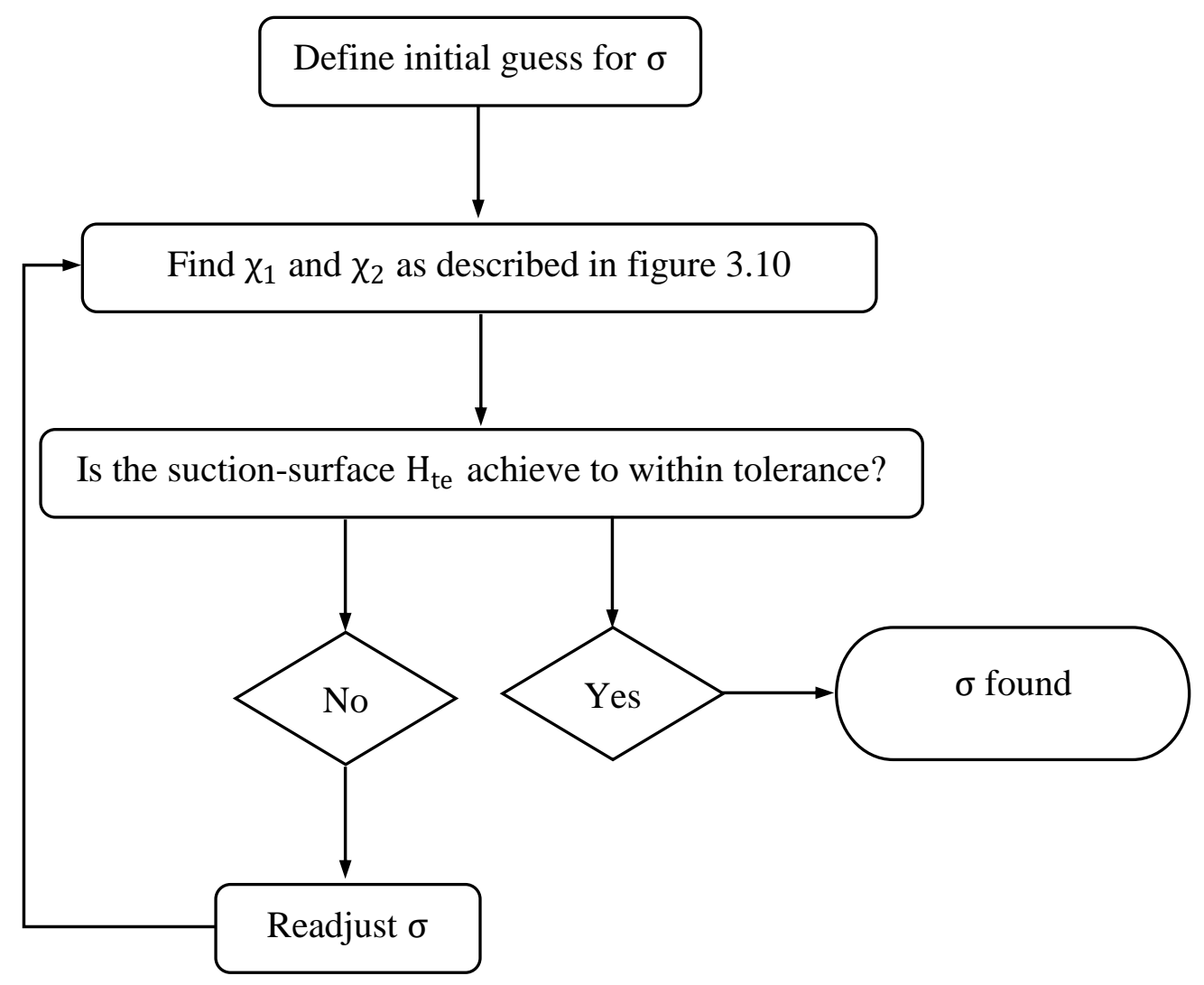

Figure 3.13: Flow chart of setting solidity based on constant trailing-edge shape factor. 
An initial solidity is first specified. For this initial solidity, $\chi_{1}$ and $\chi_{2}$ are found using the process shown Figure 3.10. The difference between the value of $\mathrm{H}_{\mathrm{te}}$ computed from MISES and the required value of $\mathrm{H}_{\mathrm{te}}=1.71$ is then calculated. If this difference is not within the required tolerance (set as 0.02), then the solidity is readjusted and the new aerofoil profile is re-calculated in MISES. This loop, shown in Figure 3.13, for adjusting $\mathrm{H}_{\mathrm{te}}$ is repeated until the required tolerance has been met.

\subsection{Linear repeating stage model}

An important part of this study is the linear repeating stage model. The linear repeating stage has two important aspects. The first is that the stage is made up of two rectilinear cascades, one for the rotor which moves at the blade speed and one for the stator which is stationary. This means that the blade speed does not vary up the span of the blade and therefore the blade profile design remains unchanged. This is important as it allows the rotational forces to be removed and then re-introduced, as discussed in the next section. The second important aspect of the model is that the stage is repeating. This is important as reaction changes the development of the endwall boundary layer in the compressor.

In a multistage compressor, the endwall boundary layers grow through the first few stages and the spanwise profile of axial velocity rapidly changes. After the first few stages, the profile settles down to a constant profile. This can be seen in the measurements taken by Smith (1970), shown in Figure 3.14, which shows how the axial velocity profile develops through a 12-stage compressor. Smith (1970) showed that in a multistage compressor the spanwise stage inlet conditions repeat after three to four stages in a wellmatched compressor. McKenzie (1997) developed this into a 'linear repeating stage' concept and it has been implemented computationally by Auchoybur \& Miller (2017) and To \& Miller (2019). The concept is designed to model an embedded compressor stage with thick endwall boundary layers. Figure 3.15 shows the computational implementation of the model used throughout this thesis. There are three elements to the implementation of this model in this thesis. 


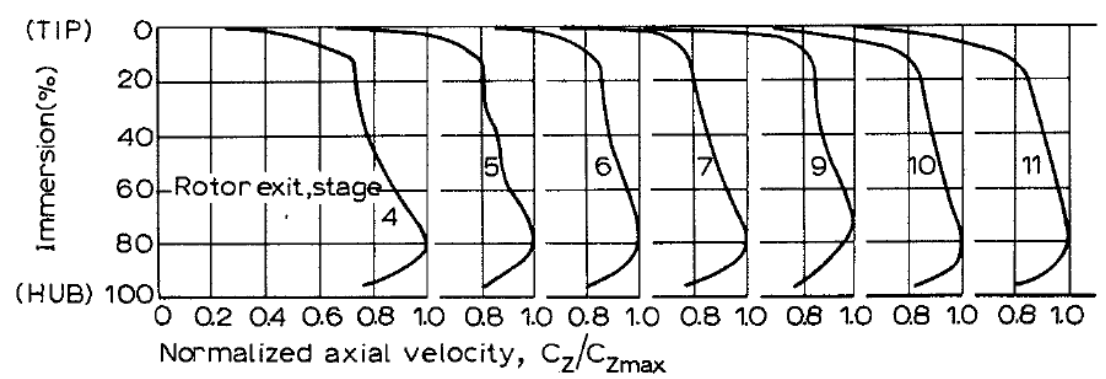

Figure 3.14: Experimental measurements from 12-stage compressor, taken from Smith (1970).

Firstly, the bulk passage effect of rotation is removed by choosing a compressor geometry which is at a span-to-radius ratio $\Delta \mathrm{r} / \mathrm{r}$ of 0 , i.e. the rotor and stator are rectilinear cascades of blades. This allows coupled-influence between the rotor and stator and removes the variation in velocity triangles up the span. This decouples the effects of the centrifugal force from the effects of changing the velocity triangle design, which is not possible in an annular compressor experiment. The value of $\Delta \mathrm{r} / \mathrm{r}$ is discussed in further detail in Section 3.5.1.

Secondly, a 1.5 stage compressor model (rotor-stator-rotor) is calculated using 3D CFD. This allows the effect of the downstream rotor on the stator flow to be modelled. The stator exit conditions are copied to the rotor inlet and every 10 calculation iterations. The spanwise distributions of stagnation pressure, stagnation temperature, radial flow angle, circumferential flow angle and the Spalart-Allmaras turbulence parameter at the stator exit, are copied to the rotor inlet. The stagnation pressure and stagnation temperature rise through the stage, therefore only the shape of the distribution is copied to the rotor inlet, according to equations 3.12 and 3.13 :

$$
\begin{gathered}
\mathrm{p}_{0, \text { in }}=\overline{\mathrm{p}}_{0, \text { in }} \frac{\mathrm{p}_{0, \text { exit }}}{\overline{\mathrm{p}}_{0, \text { exit }}} \\
\mathrm{T}_{0, \text { in }}=\overline{\mathrm{T}}_{0, \text { in }} \frac{\mathrm{T}_{0, \text { exit }}}{\overline{\mathrm{T}}_{0, \text { exit }}}
\end{gathered}
$$

where $\overline{\mathrm{p}}_{0, \text { in }}$ and $\overline{\mathrm{p}}_{0 \text {,exit }}$ are the mass-average values of $\mathrm{p}_{0, \text { in }}$ and $\mathrm{p}_{0, \text { exit }}$, and similarly for the stagnation temperatures. 


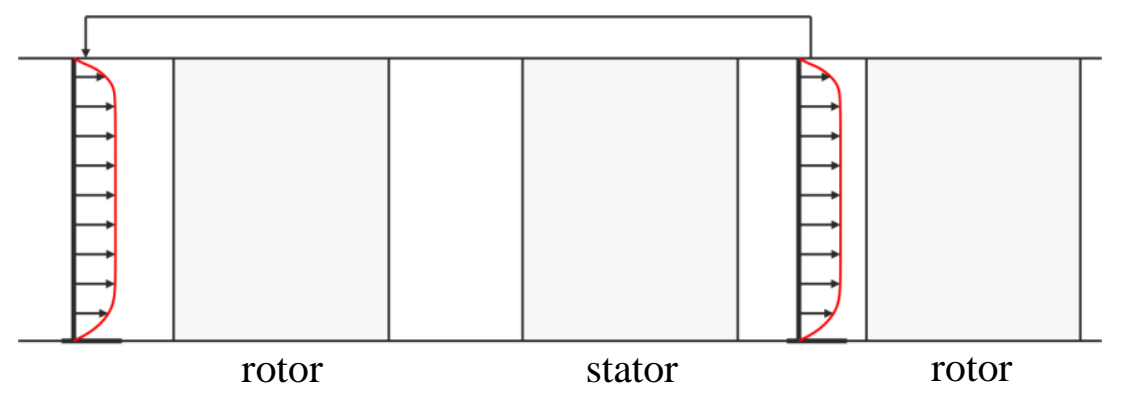

Figure 3.15: Schematic of linear repeating stage model.

Thirdly, the flow is considered to be incompressible so that the endwalls remain plane and parallel. The implication of these three elements of the model means that all streamlines experience the same rise in static-pressure since across the repeating stage. This is the same as the rise in total-pressure since the dynamic pressure is the same at the inlet and exit of the repeating stage.

The process of copying the stator exit conditions are copied to the rotor inlet is repeated until the repeating stage condition reaches a converged solution. This means that in each converged calculation the stator exit conditions are identical to the rotor inlet conditions. To achieve robust stability, particularly in calculations near to the maximum pressure rise point on a characteristic, an initially fixed uniform inlet was used for the first 15,000 calculation iterations for all cases. After 15,000 iterations, the repeating stage model was switched on for a further 15,000 iterations. An example showing the change of lost efficiency as the total number of iterations is increased is shown in Figure 3.16, at a flow coefficient which is just before the maximum pressure rise of the compressor. The lost efficiency rises sharply over the first 10,000 iterations. The change in lost efficiency over the final 1000 repeating stage iterations fluctuates by less than $0.002 \%$, as the model asymptotes to a repeating stage.

To generate the compressor characteristics presented in Chapters 5 and 6 of this thesis, the repeating stage model is used at each point along the characteristic. It was found that in all cases a maximum pressure rise was observed before the repeating stage solution started to diverge. Throughout this thesis, only calculations up to the maximum static-pressure rise coefficient are presented. 
It is clear that a significant advantage of implementing the repeating stage model in TBlock in the way described above, as opposed to modelling a full multistage compressor with repeating stages is that the smaller numerical domain for the repeating stage model is less costly. Copying the stator exit conditions to the rotor inlet every 10 calculation iterations also means that a repeating stage solution is achieved in fewer overall iterations compared to fully converging each solution after every repeat. Another advantage is that the smaller numerical domain is potentially less susceptible to cumulative numerical errors. Converged calculations, particularly near the maximum pressure rise capability of the stage, are therefore likely to be more accurate.

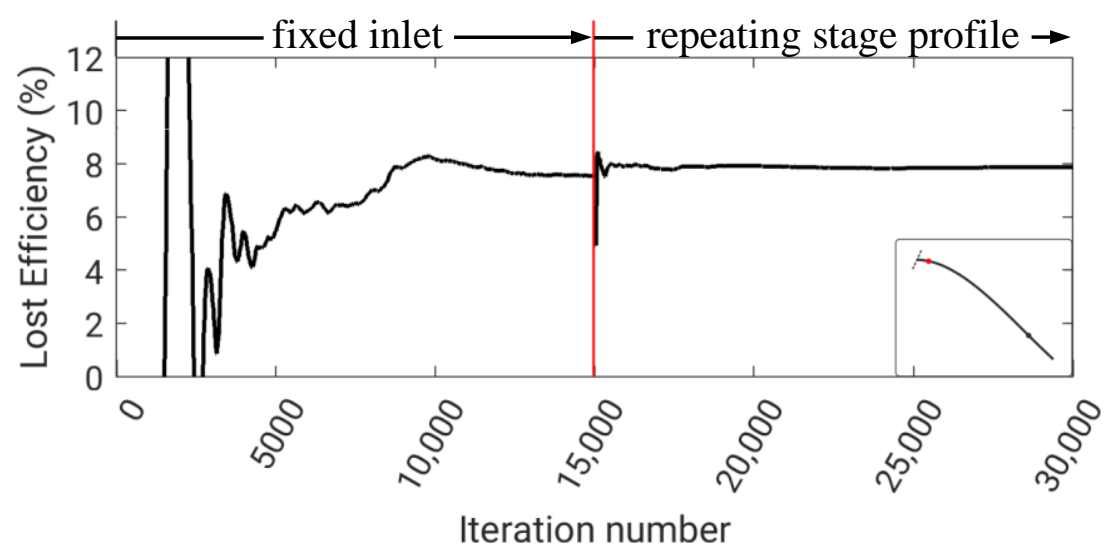

Figure 3.16: Example repeating stage convergence history.

A disadvantage to implementing the repeating stage model is that it is different to simulating a multistage compressor, where all the blade-rows communicate with each other. However, it is noted that in preliminary investigations, and found by To (2016), that results from the repeating stage model compare well with results from a multistage compressor CFD calculation.

The purpose of the repeating stage method outlined in this section is to ensure that the inlet profile is representative of an embedded stage. Although the achieved 'repeating stage' is not the same as the repeating inlet profile in an embedded stage, it provides a physical way to avoid using a fixed inlet profile as this is completely unrepresentative of the inlet profile in an embedded stage. 


\subsection{Rotation model}

A central part of this thesis is the ability to switch on and off rotational forces in a controllable way. This section is further divided into two parts. The first part looks at the effect of rotation on the stage. The second develops a model that allows the magnitude of the rotational terms to be varied.

\subsubsection{Effects of rotation}

Rotation forces have two effects on the stage, as shown in Figure 3.17. First, a bulk passage effect where the centrifugal forces are balanced by a radial pressure gradient. This causes the bulk passage flow in the rotor to move radially outward and in the stator to move radially inward. Second, a boundary layer effect due to the differential effect of centrifugal and Coriolis forces. This causes the boundary layers in the rotor to be differentially accelerated towards the casing. This effect is critical to this study as it acts as an asymmetry between the way in which the rotor and stator boundary layers develop. It was decided that the rotation model should only model the differential boundary layer effect and not the bulk passage effect. It was decided that the bulk passage effects would not be modelled as the effect causes small incidence variations up the blade span.

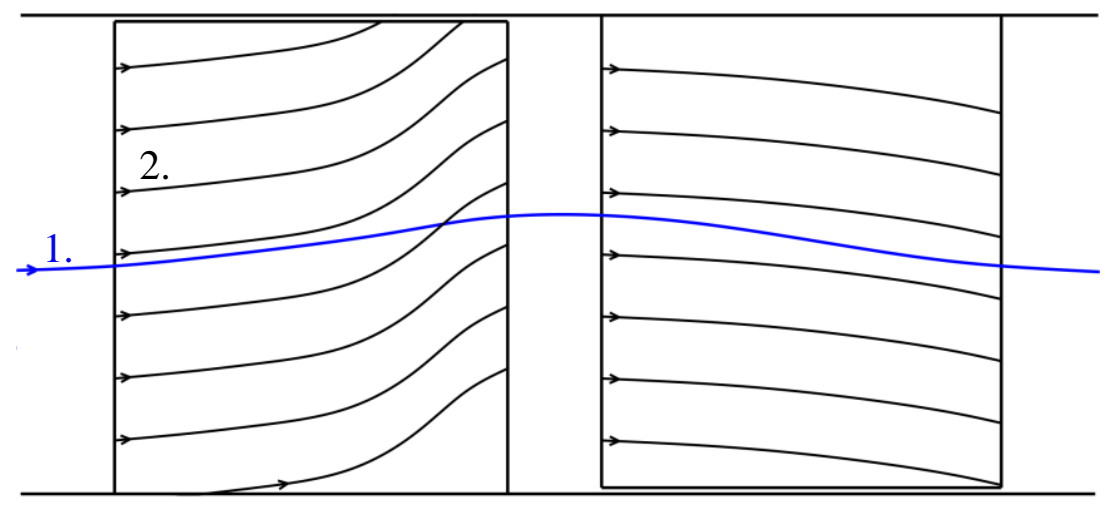

Figure 3.17: Schematic showing two effects of rotation: (1) bulk passage effect on freestream streamline; and (2) differential boundary layer effect on rotor and stator boundary layer streamlines. 
In a real design the blade profile would be varied along its span to compensate for this effect. In this controlled study, detailed redesign of the blade across the span must be avoided and so it was decided that the bulk passage effects of rotation would not be modelled.

To model only the differential boundary layer effect, we first remove both effects of rotation by choosing a compressor geometry which is at a span-to-radius ratio, $\Delta \mathrm{r} / \mathrm{r}$, of 0 , i.e. the rotor and stator are rectilinear cascades of blades. The rotational terms were removed by creating the stage from two rectilinear cascades, as discussed in Section 3.4.

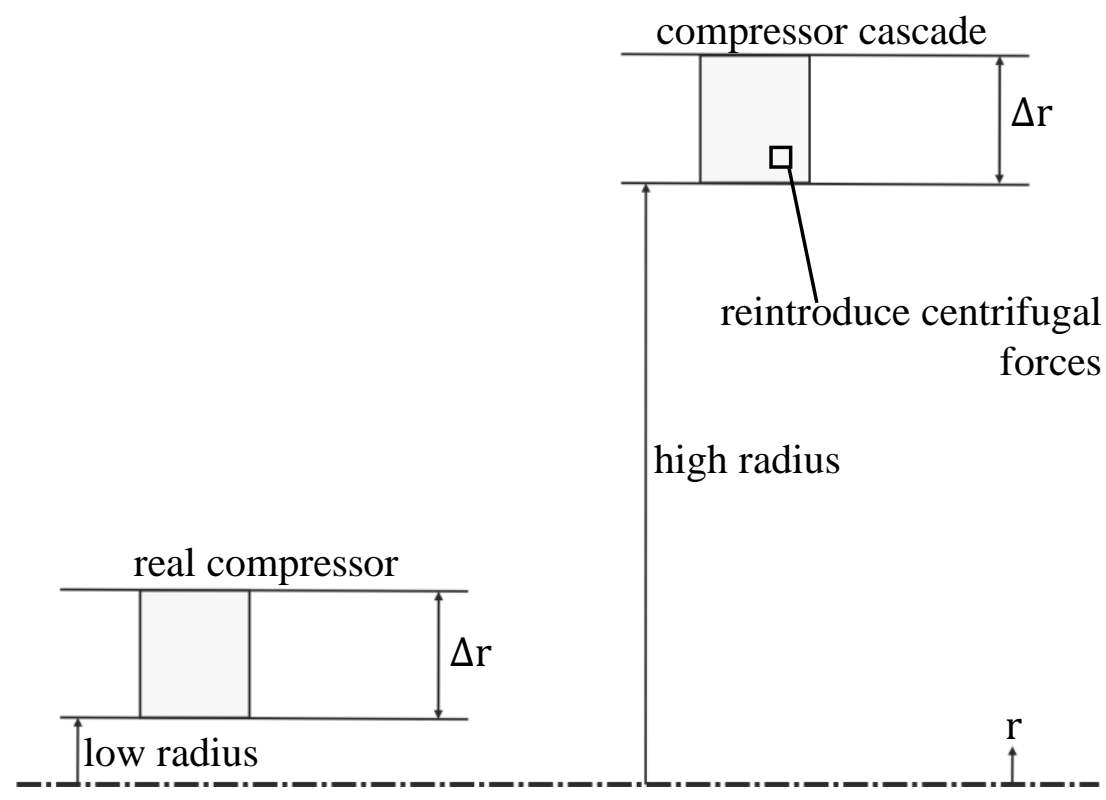

Figure 3.18: Real compressor (left) and compressor cascade (right) with rotation model.

To achieve $\Delta \mathrm{r} / \mathrm{r}$ close to zero, the compressor is moved to high radius, as shown in Figure 3.18. For an aspect ratio of 2 , the flow was observed to be 2-D below a value of $\Delta r / r$ equal to approximately $2.9 \times 10^{-3}$. The radius of the compressor was set to achieve a value of $\Delta \mathrm{r} / \mathrm{r}$ equal to $2.5 \times 10^{-3}$.

\subsubsection{Reintroduction of differential rotational forces}

To reintroduce only the differential boundary layer effect, the rotation model added a body force per unit volume into the CFD calculation of form: 


$$
\frac{\rho V_{\theta}^{2}}{r}-\left.\frac{\overline{\rho V_{\theta}^{2}}}{r}\right|_{(x, r)}
$$

This new term represents the perturbation centrifugal forces. The first term is the value of the centripetal acceleration $V_{\theta}^{2} / r$ at each meridional location. The second term is defined as the pitchwise volume-averaged value of $V_{\theta}^{2} / r$ at the same meridional position i.e. the same axial and radial coordinates and $r$ is an effective radius. An example calculation at a single meridional position is shown in Figure 3.19. The new model was introduced into TBlock as a source term, described by equation 3.15:

$$
\rho \frac{D V_{r}}{D t}-\rho\left(\frac{V_{\theta}^{2}}{r}-\left.\frac{\overline{V_{\theta}^{2}}}{r}\right|_{(x, r)}\right)=-\frac{\partial p}{\partial r}+\operatorname{viscous}+F_{r}
$$
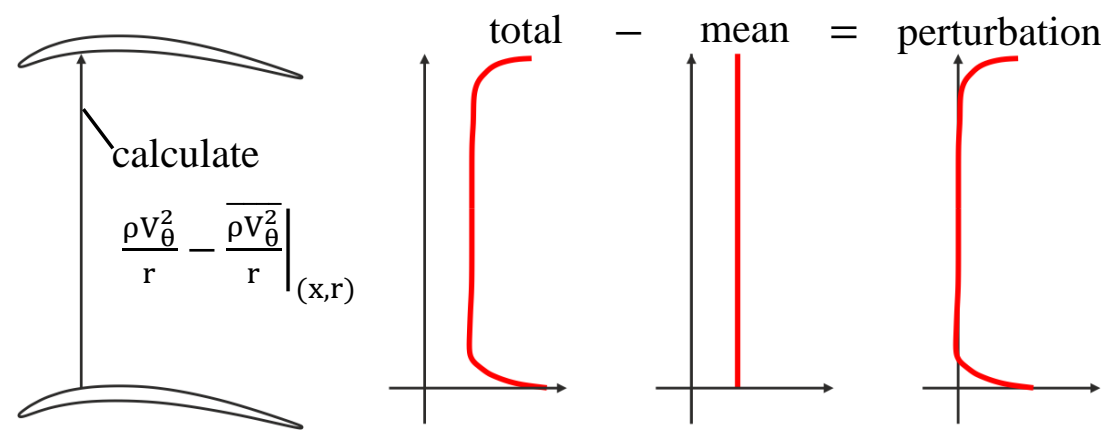

Figure 3.19: Example perturbation centrifugal force calculation extracted from the results computed in Chapter 6.

The source term was updated everywhere in the flow every 5 calculation iterations. This increased the computation time by less than $6 \%$ (from $\sim 18$ hours to $\sim 19$ hours).

The model allows an effective radius to be set. By setting a relatively high value, the blades act as if part of a rectilinear cascade of blades. Setting the effective radius equal to the radius of a real compressor would introduce the differential boundary layer effects of rotational that are expected in a real compressor. The benefit of using the model, rather 
than changing the real radius of the compressor, is that the geometry of the stage remains unchanged as the magnitude of the body force is varied.

To generate the compressor characteristics presented in Chapter 6 of this thesis, the rotation model is switched on and the repeating stage model is used, at each point along the characteristics.

It is clear that a significant advantage of implementing the rotation model in TBlock using a structured H-mesh topology is that interpolation is not required to calculate the perturbation centrifugal forces at each position. It is expected that the advantages of using a more sophisticated structured grid topology, are disadvantaged by errors in interpolation. Another advantage of this implementation of the rotation model is that as the effective radius is set to be much less than the true radius, the magnitude of the centrifugal effect in the boundary layers is increased but the average effect remains zero. This can be understood by looking at the form of equation 3.14 .

It should be noted that the purpose of the rotation method outlined in this section is to ensure that the modelling of the perturbation centrifugal forces captures the differential effect of rotation in a physical way. This effect is critical to this study, acting as an asymmetry between the way in which the rotor and stator boundary layers develop. 


\section{Chapter 4}

\section{Profile loss}

The total loss across a compressor stage is typically decomposed into two parts, the loss associated with the blade profile and the loss associated with the endwall flow. In this chapter, we begin by examining the effect of reaction on profile loss. This Chapter uses the CFD setup described in Section 3.1.1. Conventional levels of design flow coefficient $\left(\Phi_{d}=0.597\right)$ and work coefficient $\left(\Psi_{d}=0.436\right)$ are fixed throughout this chapter, except in Section 4.4 where the effect of varying $\Phi_{\mathrm{d}}$ and $\Psi_{\mathrm{d}}$ is examined.

The chapter is divided into six parts. The first part develops a new framework through which we can understand the effect of reaction on the profile loss of a stage. In the second part this framework is used with the solidity held constant. In the third part the solidity is varied as the reaction is changed. The forth part looks at the effect of reaction across the Smith Chart. The fifth part investigates the incidence range of the compressor as the reaction is changed. At the end of the chapter, there is a summary.

\subsection{Framework of lost efficiency}

The lost efficiency of a stage due to profile loss alone can be written as:

$$
\frac{\mathrm{T} \Delta \mathrm{s}}{\Delta \mathrm{h}_{0}}=\frac{(\mathrm{T} \Delta \mathrm{s})_{\text {rotor }}+(\mathrm{T} \Delta \mathrm{s})_{\text {stator }}}{\Delta \mathrm{h}_{0}}
$$


where $\mathrm{T}$ is the exit static-temperature of the stage and $\Delta \mathrm{h}_{0}$ is the rise in specific stagnation-enthalpy across the stage. The total entropy generation in the attached boundary layer on each blade, either rotor or stator, can be calculated by integrating the entropy production in the boundary layer over the blade surface:

$$
\dot{S}=\sum \mathrm{c}\left[\int_{0}^{1} \mathrm{C}_{\mathrm{d}} \rho \frac{\mathrm{V}_{0}^{3}}{\mathrm{~T}} \frac{\mathrm{dx}}{\mathrm{c}}\right]
$$

where the summation is across both blade surfaces and $V_{0}$ is the velocity at the boundary layer edge. Writing equation 4.2 in the form of the lost efficiency of a row of blades gives:

$$
\begin{aligned}
& \left(\frac{\mathrm{T} \Delta \mathrm{s}}{\Delta \mathrm{h}_{0}}\right)_{\text {row }}=2 \frac{\mathrm{C}_{0}}{\mathrm{~V}_{\mathrm{x}}} \sum \underbrace{\sigma}_{\text {1. Solidity }} \underbrace{\left(\frac{\mathrm{V}_{\text {in,ref }}}{\mathrm{C}_{0}}\right)^{3}}_{\text {2. Velocity }} \underbrace{\left[\int_{0}^{1} \mathrm{C}_{\mathrm{d}}\left(\frac{\mathrm{V}_{0, \text { ref }}}{\mathrm{V}_{\text {in,ref }}}\right)^{3} \frac{\mathrm{dx}}{\mathrm{c}}\right]}_{\text {. Single blade }} \\
& \text { triangle term loss coefficient }
\end{aligned}
$$

where we follow Denton (1993) and write the enthalpy change in terms of an isentropic stage reference velocity $\mathrm{C}_{0}$ :

$$
\mathrm{C}_{0}=2 \sqrt{\Delta \mathrm{h}_{0}}
$$

and $V_{\text {in,ref }}$ is the relative inlet velocity into the blade row.

The lost efficiency given by equation 4.3 , is made up of three terms. The first term, the solidity, represents the effect of changing the number of blades in a row. The second term represents the effect of changing the velocity triangle on loss. This term is high for a blade row with a high relative inlet velocity. This term shows that one of the key aims for a designer is to minimise the ratio of the cube of the relative inlet velocity into the blade rows relative to the enthalpy rise of the stage. The third term is the single blade loss coefficient. This term is high if a blade has a high surface velocity, relative to the blade inlet velocity, or a large wetted area. In a repeating stage, the term $C_{0} / V_{x}$ is a function of the flow coefficient $\Phi$ and work coefficient $\Psi$ only. 
In the following three sections, the three terms in equation 4.3 will be used as a framework through which we can understand the effect of reaction on the lost efficiency of a stage. It will be shown that changing reaction changes all three terms. Only by controlling how these terms change with reaction can the effect of reaction on the lost efficiency of a stage be understood.

\subsection{Constant solidity}

It is commonly believed that compressors of $50 \%$ reaction have the highest stage efficiency. This way of thinking is based on the idea that the blade solidity is held constant. The lost efficiency of each blade row for the case of constant solidity is plotted in Figure 4.1. The shape of each line is mainly determined by changes in term 2 in equation 4.3 , the velocity triangle term.

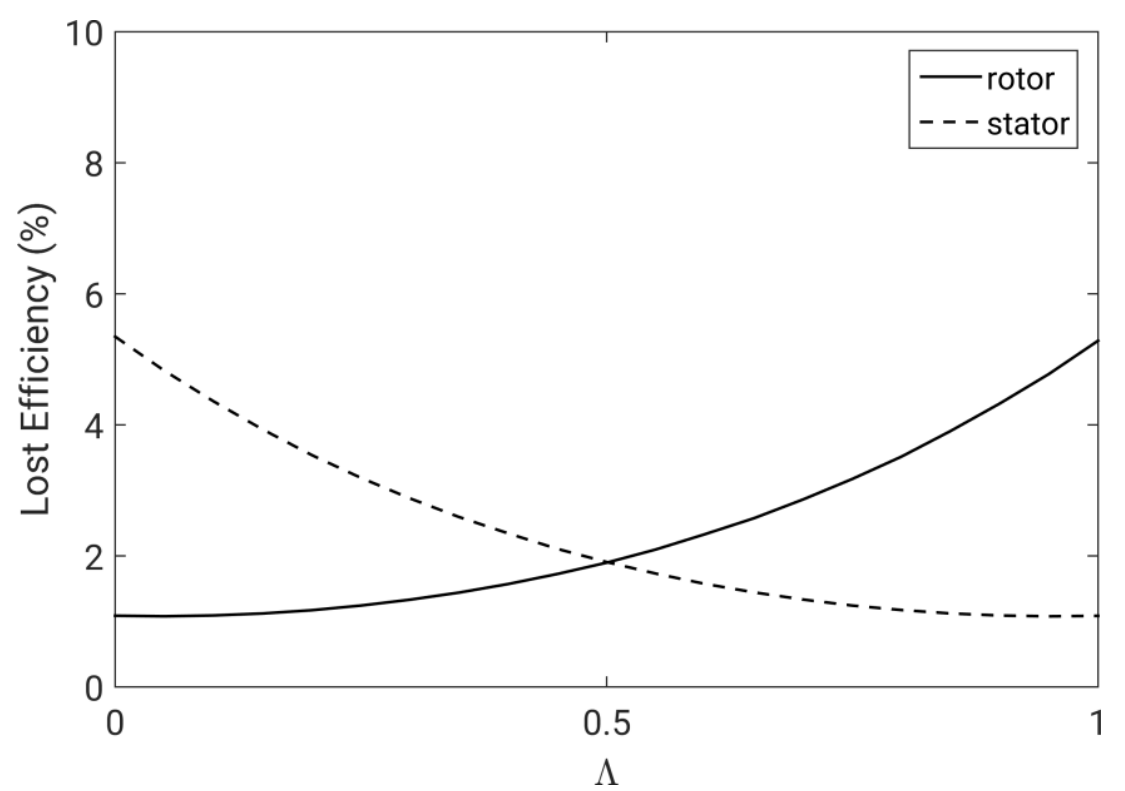

Figure 4.1: Blade row lost efficiency for the case of constant solidity and blade loss coefficient (MISES).

Moving from $50 \%$ to $70 \%$ reaction, term 2 changes by approximately $+60 \%$ and term 3 changes by $-7 \%$. The shape of the lines is caused by the way in which the velocity triangle 
controls the relative inlet velocity $\mathrm{V}_{\mathrm{in} \text {,ref }}$ into each blade row, and the fact that loss scales with the cube of the relative inlet velocity into the blade row. The black line in Figure 4.2 shows the lost efficiency of this stage. It is clear that $50 \%$ reaction must be the most efficient stage because it minimizes the sum of $V_{i n, r e f}^{3}$ into both blade rows. The variation in lost efficiency is symmetrical about 50\% reaction. Increasing reaction from $50 \%$ to $70 \%$ reduces the stage efficiency by $0.39 \%$.

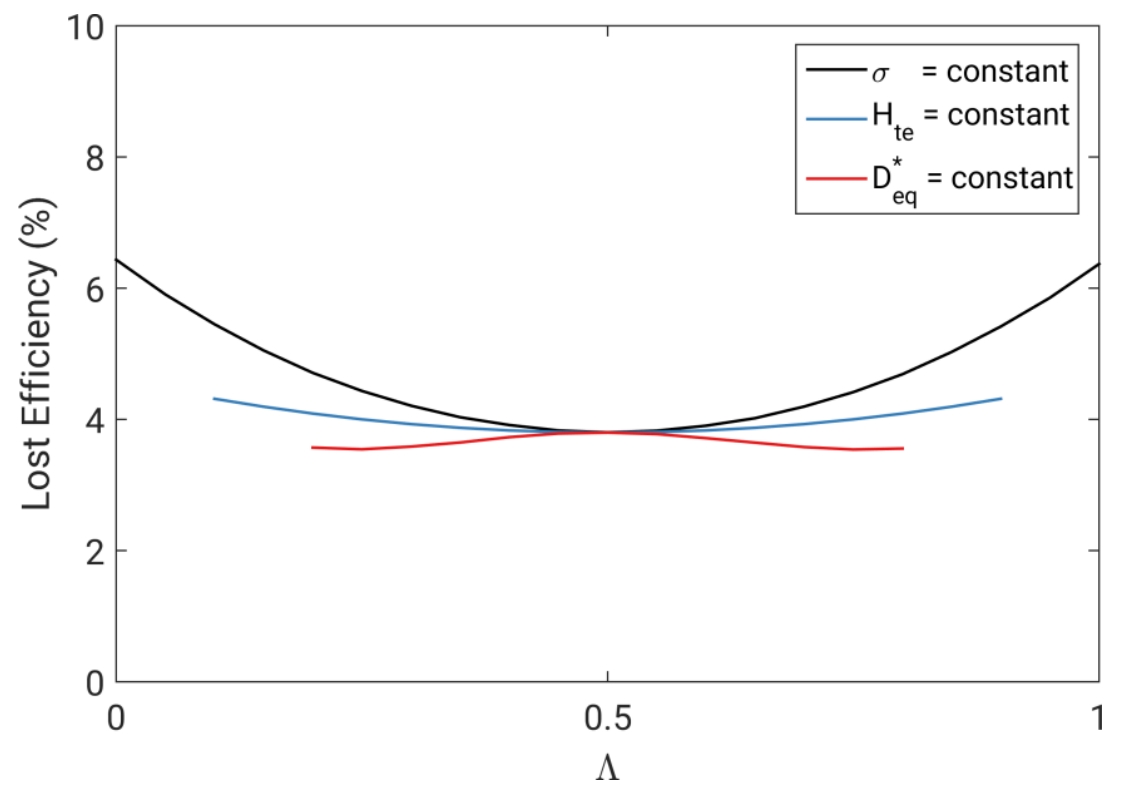

Figure 4.2: Stage lost efficiency for the cases of constant solidity, $\mathrm{D}_{\mathrm{eq}}^{*}$ and $\mathrm{H}_{\mathrm{te}}$ (MISES).

\subsection{Effect of varying solidity}

The view developed in the previous section was the view of the author until he received the personal communication from LHS quoted in Section 1.1. In this section the solidity is controlled in two ways. First, as LHS proposed, the Equivalent Diffusion Ratio $D_{\text {eq }}^{*}$ of each blade row is held constant. Second, the shape factor of the suction-surface boundary layer at the trailing-edge $\mathrm{H}_{\text {te }}$ of each blade row is held constant.

The effect of holding the equivalent diffusion ratio $\mathrm{D}_{\mathrm{eq}}^{*}$ constant and equal to 1.78 is shown as the red line in Figure 4.2. The change in the solidity is shown in Figure 4.3. The red line in Figure 4.2 shows that, as LHS said, 50\% reaction is now the most 
inefficient compressor. In fact, at 50\% reaction the compressor has an efficiency which is $0.22 \%$ lower than an equivalent compressor at $70 \%$ reaction. Figure 4.3 shows that this is caused by dropping the solidity in both the rotor and stator by approximately $60 \%$. It seems surprising that the solidity in both blade rows drops simultaneously. This effect will be explained later in the section.

The effect of holding the shape factor of the suction-surface boundary layer at the trailing edge $\mathrm{H}_{\text {te }}$ constant is shown as the blue line in Figure 4.2. The line shows that the change in lost efficiency is almost independent of reaction. Increasing reaction from $50 \%$ to $70 \%$ reduces the stage efficiency by only $0.13 \%$. Figure 4.3 shows that increasing reaction from $50 \%$ to $70 \%$ reduces the solidity in both blade rows by approximately $30 \%$.

It is clear that as LHS said, solidity plays an important role in determining the impact of reaction on compressor efficiency. However, to understand this effect, a choice must be made about how the solidity is varied as the design of the blade is changed.

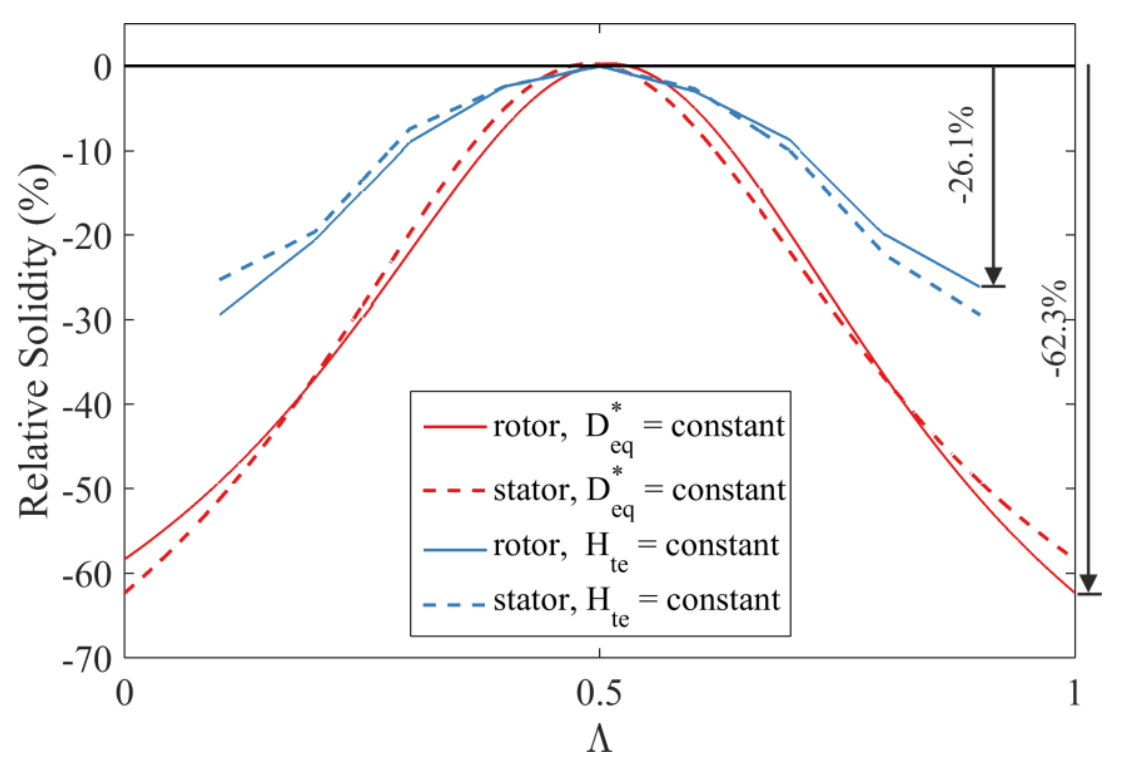

Figure 4.3: Reduction in solidity relative to $50 \%$ reaction for the cases of constant $\mathrm{D}_{\mathrm{eq}}^{*}$ and $\mathrm{H}_{\mathrm{te}}(\mathrm{MISES})$. 
To understand why there is a reduction in rotor and stator solidity either side of $50 \%$ reaction, first consider the Diffusion Factor DF equation, developed by Lieblein (1953), in its simplest form:

$$
\mathrm{DF}=1-\mathrm{DH}+\frac{\Delta \mathrm{V}_{\theta}}{2 \mathrm{~V}_{\mathrm{in}, \mathrm{ref}} \sigma}
$$

where DH is the blade row de Haller number, $\Delta \mathrm{V}_{\theta}$ is the change in absolute tangential velocity across the blade row and $V_{\text {in,ref }}$ is the relative inlet velocity into the blade row. Equation 4.5 rearranges to:

$$
\sigma=\frac{\Delta \mathrm{V}_{\theta} / 2 \mathrm{~V}_{\text {in, ref }}}{\mathrm{DF}-(1-\mathrm{DH})}
$$

from which the solidity $\sigma$ can be calculated. We can then define the top and bottom of equation 4.6 as two terms, given by equations 4.7 and 4.8 :

$$
\text { term } 1=\Delta V_{\theta} / 2 V_{\text {in,ref }}
$$

and

$$
\text { term } 2=\mathrm{DF}-(1-\mathrm{DH})
$$

Term 1 represents a loading term relative to the relative inlet velocity into the blade row. Term 2, for a fixed diffusion factor DF equal to 0.45 , is proportional to the blade row de Haller number DH. Figures 4.4 and 4.5 show the variation of term 1 and term 2, defined by equations 4.7 and 4.8 , relative to their values at $50 \%$ reaction for the rotor and stator respectively. The maximum solidity occurs when the two lines meet at a tangent. This occurs at approximately $50 \%$ reaction.

Term 1, in Figure 4.4, varies almost linearly with reaction. The reason for this is that the work coefficient and blade speed are constant so that $\Delta \mathrm{V}_{\theta}$ is constant. However, 
as the reaction rises, the relative inlet velocity into the rotor $V_{\text {in,ref }}$ rises. This steady rise in $V_{\text {in,ref }}$ causes the approximately linear rise in term 1.

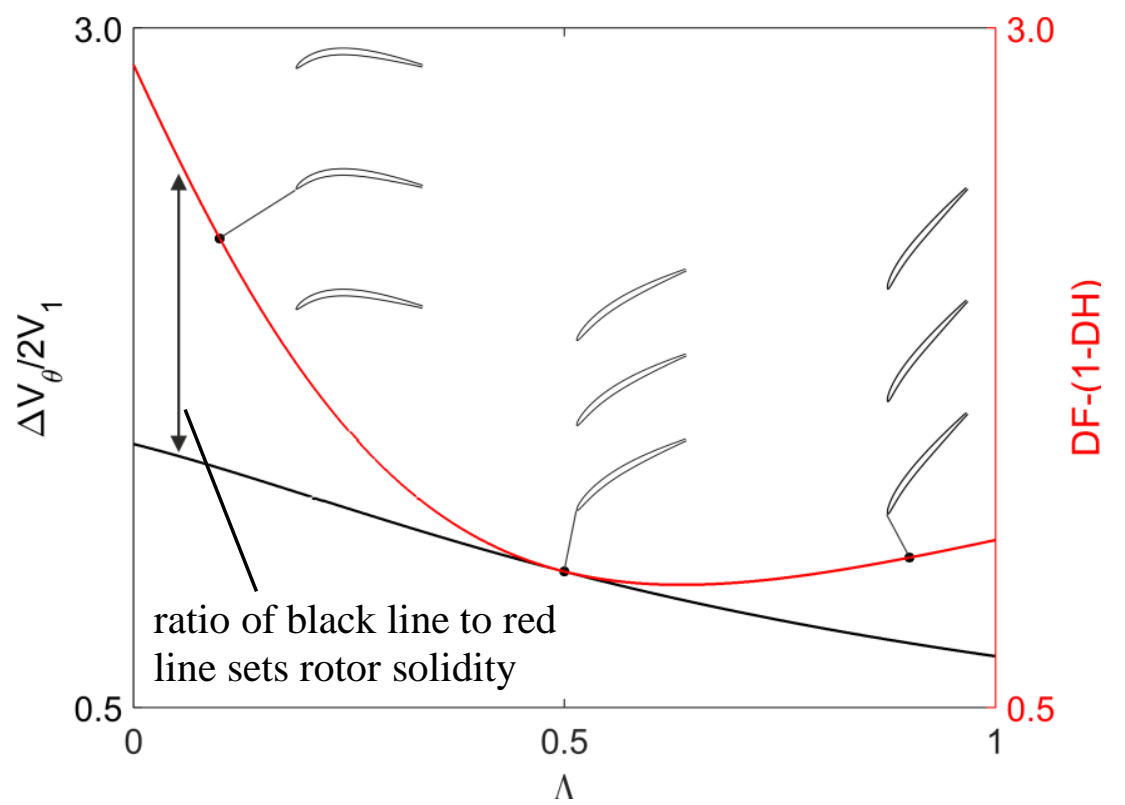

Figure 4.4: Change in term 1 (equation 4.7) and term 2 (equation 4.8) relative to 50\% reaction for the rotor.

Term 2, in Figure 4.4, varies almost parabolically with reaction. This variation is driven by the variation in de Haller number DH. One might expect that as reaction is increased, the rotor DH would continually drop, but it does not. Above a reaction of approximately $60 \%$ it starts to rise again. This is because as reaction rises, the rotor static-pressure rise continually increases, however $\mathrm{V}_{\text {in,ref }}$ also increases. The two effects combine to set $\mathrm{DH}$. As reaction increases beyond approximately 60\%, the increase in static-pressure rise across the rotor is weak relative to the increase in $V_{\text {in,ref }}$. This results in the de Haller number rising. For reference, the corresponding rotor and stator aerofoil profiles are shown in Figure 4.4 and Figure 4.5 for 10\%, 50\% and 90\% reaction.

The inflection point in term 2 is at a reaction of approximately $60 \%$, however, the gradient of term 1 results in the two lines meeting at a tangent at approximately $50 \%$ reaction. Looking once again at Figure 4.3 we can see that for the case of constant $\mathrm{D}_{\mathrm{eq}}^{*}$ and constant $\mathrm{H}_{\text {te }}$ the maximum solidity occurs close to, but not quite at, 50\% reaction. 


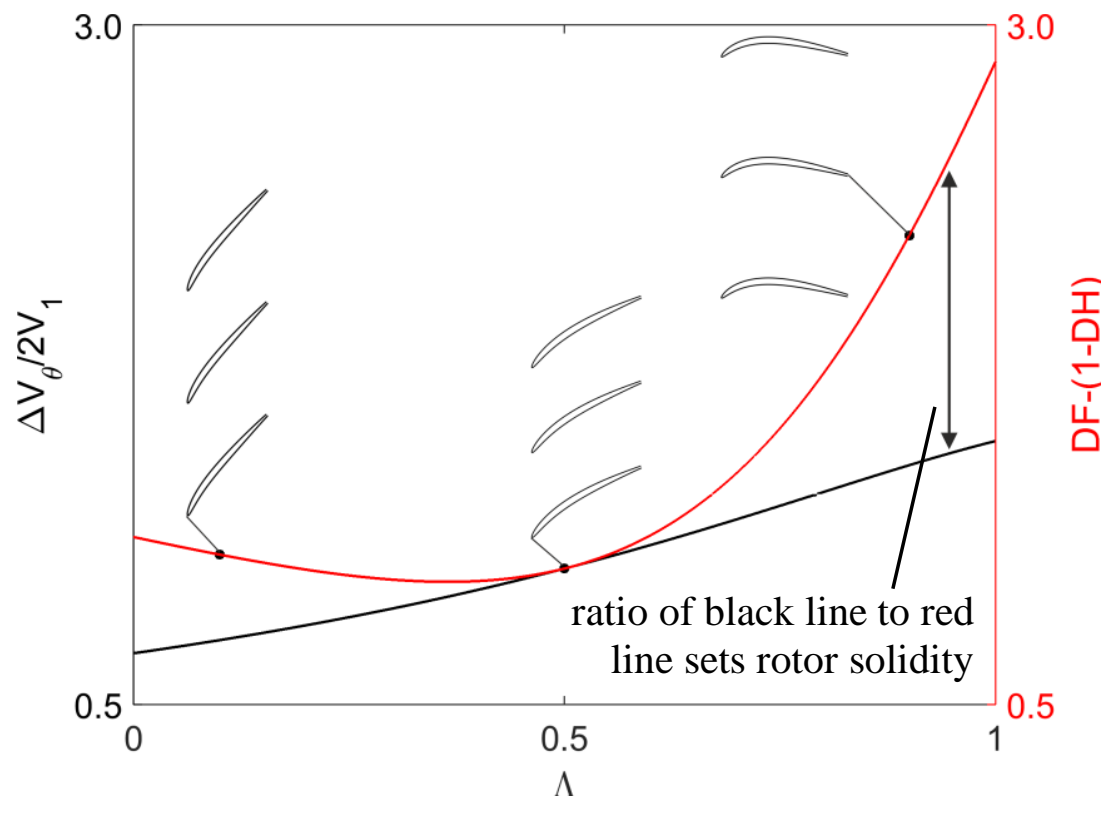

Figure 4.5: Change in term 1 (equation 4.7) and term 2 (equation 4.8) relative to 50\% reaction for the stator.

\subsection{Effect of work and flow coefficient}

It is clear from the previous section that whether $50 \%$ reaction is the most, or least, efficient compressor depends on a trade between the solidity effect (term 1 in equation 4.3) and the velocity triangle and blade loss coefficient effects (term 2 and term 3 in equation 4.3). This trade depends on the particular work and flow coefficient at which the compressor is designed.

The Smith Charts in Figures 4.6 and 4.7 show the effect of the work and flow coefficient on whether $50 \%$ reaction is the most, or least, efficient compressor. Figure 4.6 shows the case of constant solidity and Figure 4.7 shows the case of constant $\mathrm{D}_{\text {eq }}^{*}$. The contours show the difference in the efficiency between a compressor of $70 \%$ reaction and a compressor of $50 \%$ reaction, given by

$$
\Delta \eta=\eta_{\Lambda=70 \%}-\eta_{\Lambda=50 \%}
$$


Blue means that $50 \%$ reaction is most efficient and red means that $50 \%$ reaction is the least efficient.

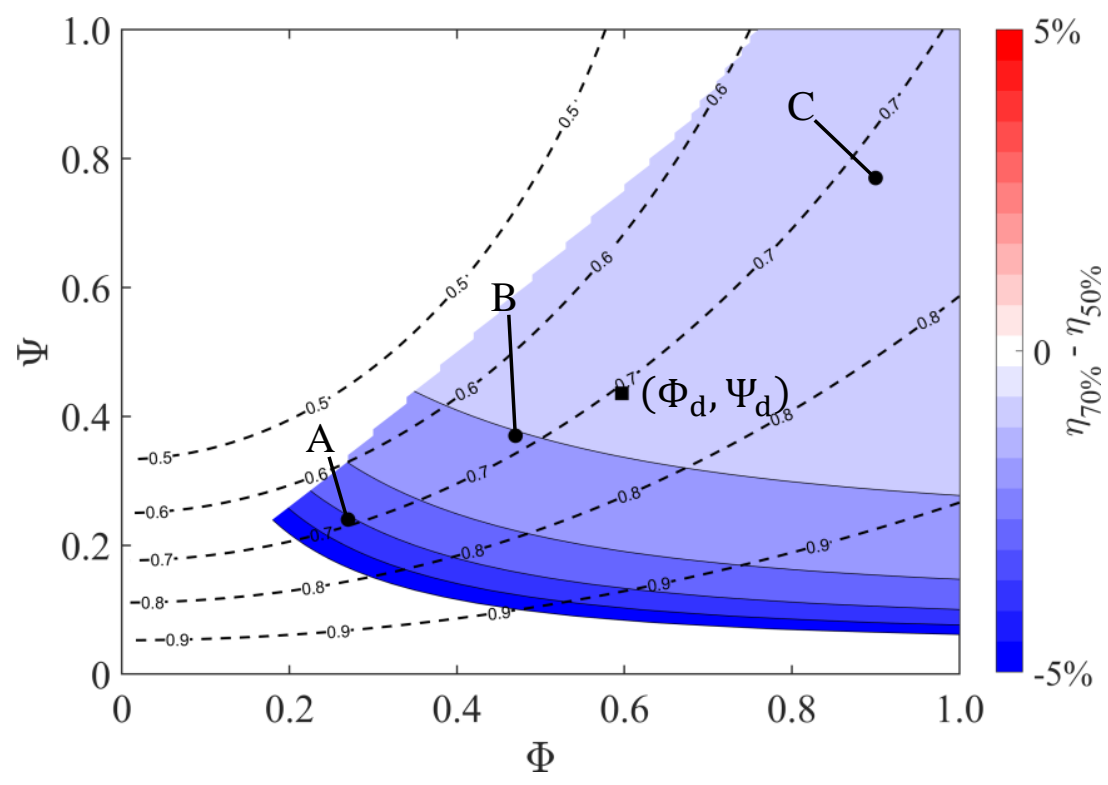

Figure 4.6: Smith Chart showing the effect of reaction on efficiency for the case of constant solidity. The dashed lines are lines of constant de Haller number at $50 \%$ reaction.

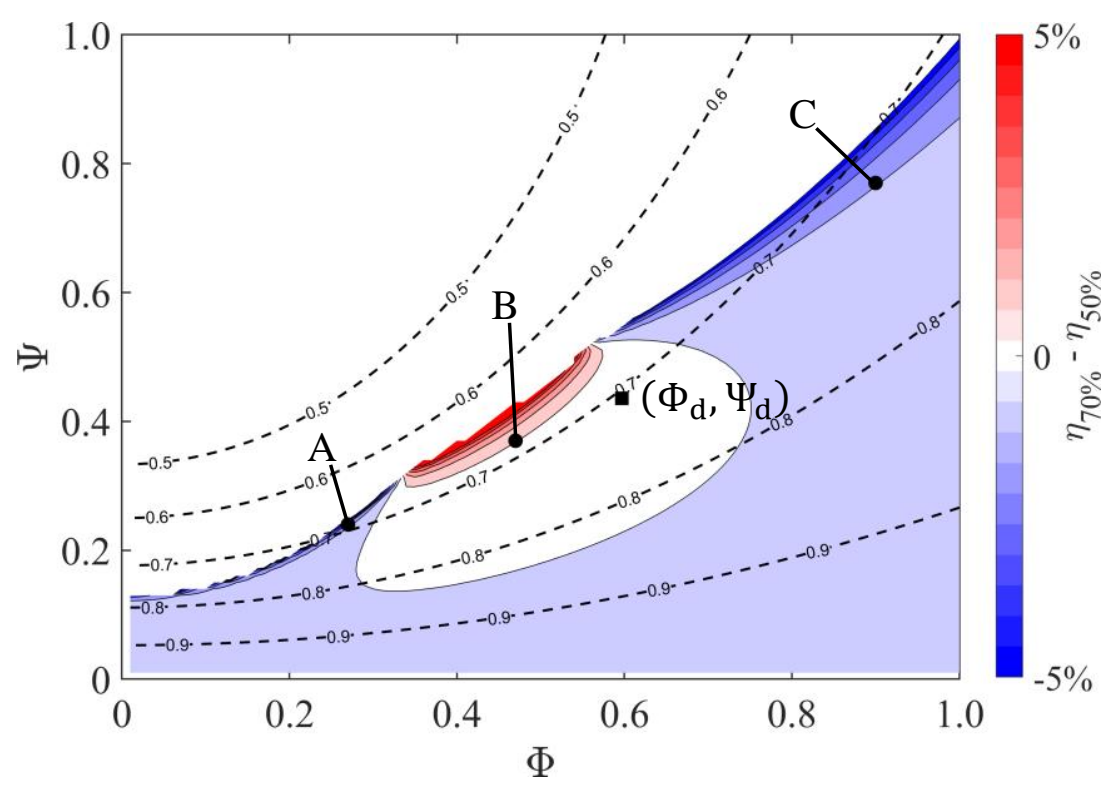

Figure 4.7: Smith Chart showing the effect of reaction on efficiency for the case of constant $\mathrm{D}_{\mathrm{eq}}^{*}$. The dashed lines are lines of constant de Haller number at $50 \%$ reaction. 
The variation of lost efficiency with reaction at the three points A, B and C in both Figures 4.6 and 4.7 are shown in Figure 4.8. Section 2.1.2 explains how the lost efficiency is calculated for these cases. The black dotted lines are lines of constant de Haller number at $50 \%$ reaction.
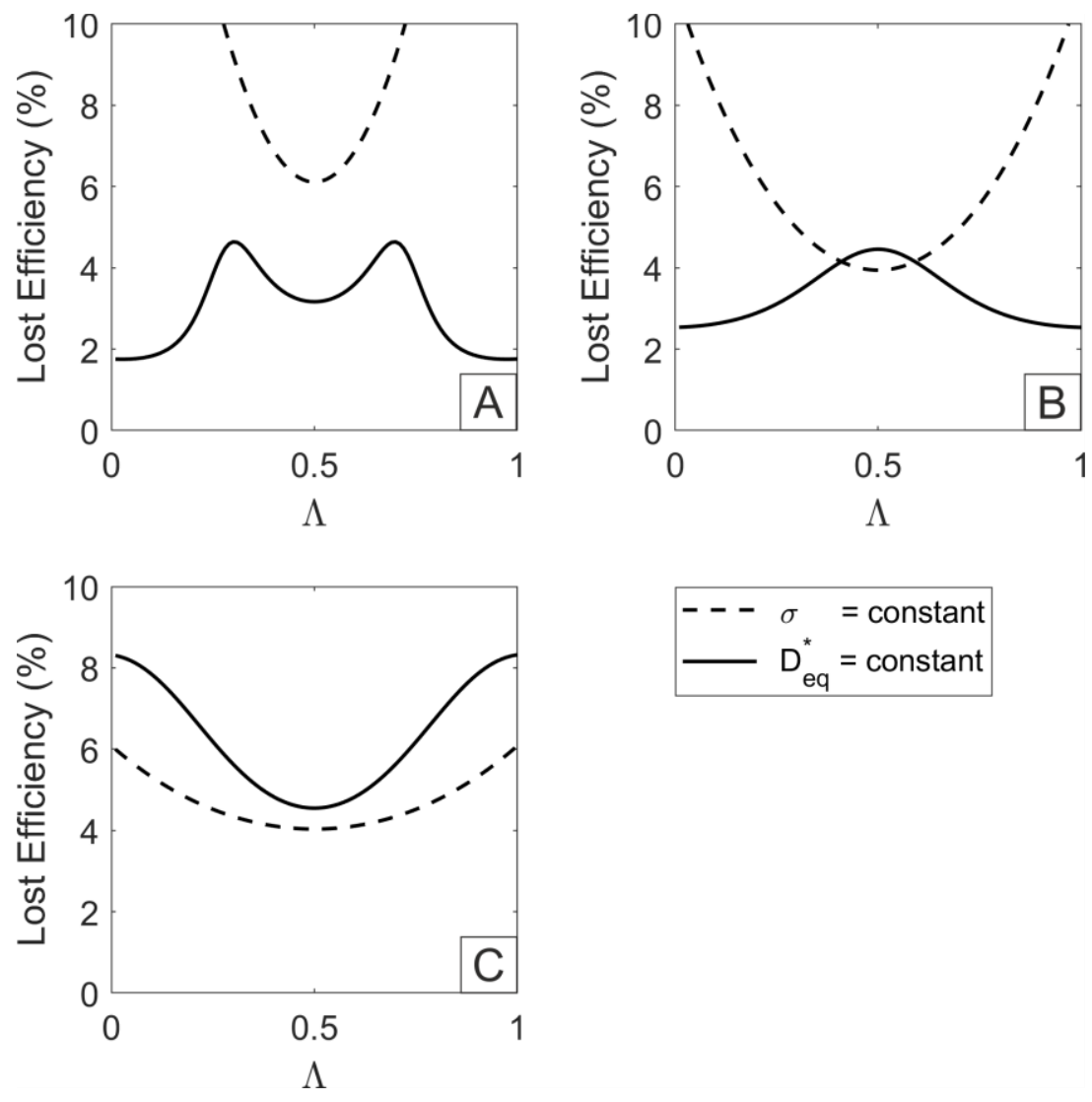

Figure 4.8: Variation of stage lost efficiency with reaction at points $\mathrm{A}, \mathrm{B}$ and $\mathrm{C}$ in Figures 4.6 and 4.7.

From Figures 4.6 and 4.7 a number of points can be made. First, as the work and flow coefficient are raised, point $\mathrm{C}$, in both Smith Charts, is a blue region. This implies that in this region the $50 \%$ reaction compressor is more efficient than the $70 \%$ reaction compressor by approximately $0.31 \%$ for the case of constant solidities and $1.08 \%$ in the case of constant $\mathrm{D}_{\mathrm{eq}}^{*}$. Second, the region close to the work and flow coefficient explored earlier in this paper, point $\mathrm{B}$, the effect of reaction is very sensitive to how the designer selects solidity. By changing the way the solidity is set, $50 \%$ reaction can switch between 
the most, and the least, efficient compressor. Finally, as the work and flow coefficient are dropped, point A, both Smith Charts once again show a blue region. This shows that the $50 \%$ reaction compressor is more efficient than the $70 \%$ reaction compressor. In fact, for the case of constant solidity, a 50\% reaction compressor is shown to be approximately $3.11 \%$ more efficient than a $70 \%$ reaction compressor.

\subsection{Incidence range}

This previous sections have highlighted that whether $50 \%$ reaction is the most, or least, efficient compressor depends on a trade between the solidity effect (term 1 in equation 4.3) and the velocity triangle and blade loss coefficient effects (term 2 and term 3 in equation 4.3). This trade is also critical in determining the effect of reaction on the 2-D operating range of the compressor. To assess how reaction affects the operating range of the compressor, the boundary layer trailing-edge shape factor $\mathrm{H}_{\mathrm{te}}$, and the blade row incidence ranges are considered, in turn.

Figure 4.9 shows how the shape factor of the suction-surface boundary layer at the trailing-edge $\mathrm{H}_{\mathrm{te}}$ changes with reaction, for the case of constant solidity and constant Equivalent Diffusion Ratio $\mathrm{D}_{\mathrm{eq}}^{*}$. It is clear that setting $\mathrm{D}_{\mathrm{eq}}^{*}$ constant is undesirable because as the reaction moves away from 50\% the boundary layers are driven toward separation. Equally it is clear that holding the solidity constant is undesirable because as the reaction moves away from $50 \%$ the stage becomes over-bladed.

Figure 4.10 shows how the range of efficient operation of the rotor changes with reaction, for the case where the solidity is set by a constant value of $\mathrm{H}_{\mathrm{te}}$ at the design point. It is clear that as reaction rises, setting $\mathrm{H}_{\mathrm{te}}$ results in a narrower range of efficient operation. In all cases it was observed that a critical level of negative incidence exists, at which the suction-surface boundary layer switched from an attached to a separated state. Beyond this critical level, there is a sharp rise in lost efficiency. This occurs at $-12.5^{\circ}$ for the $50 \%$ reaction rotor compared to $-10.5^{\circ}$ for the $70 \%$ reaction rotor. The locus of this switch from attached to separated flow is shown as the red line in Figure 4.10. The open red markers indicate where there was a clear change in the MISES calculation from 
attached to separated flow. The closed red markers indicate where a separated solution could not be converged.

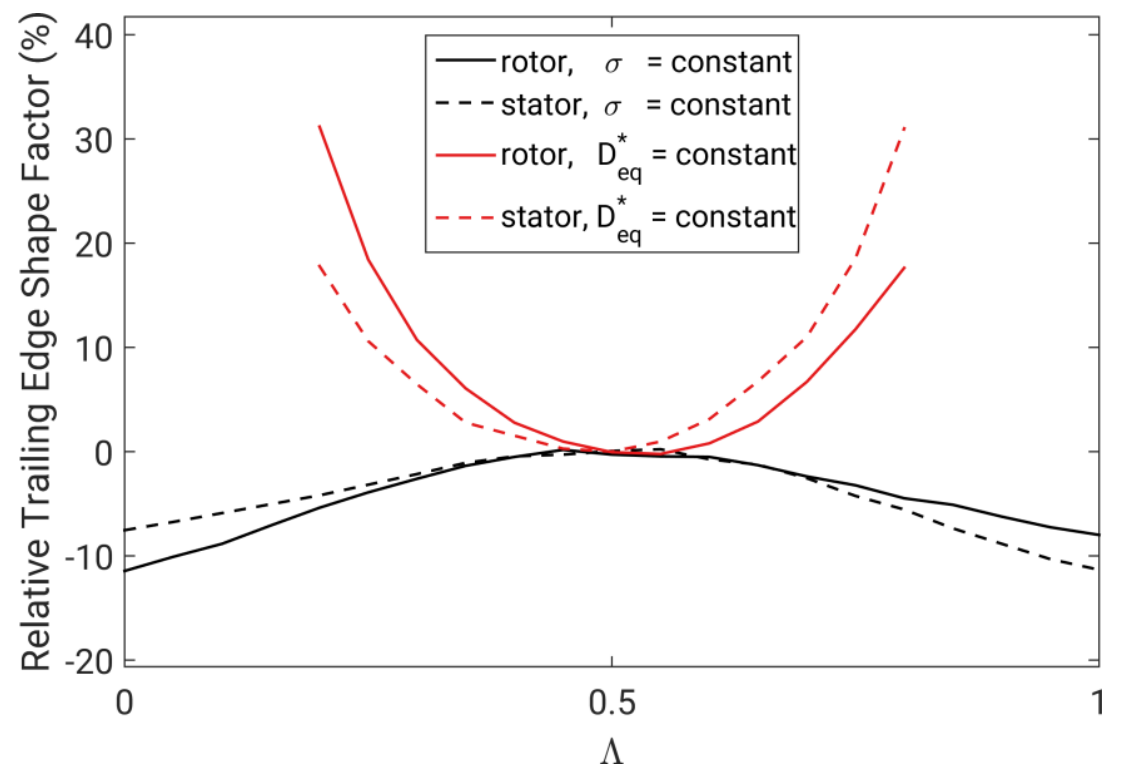

Figure 4.9: Change in suction-surface trailing edge shape factor relative to $50 \%$ reaction for the cases of constant solidity and solidity set by constant $\mathrm{D}_{\mathrm{eq}}^{*}$ (MISES).

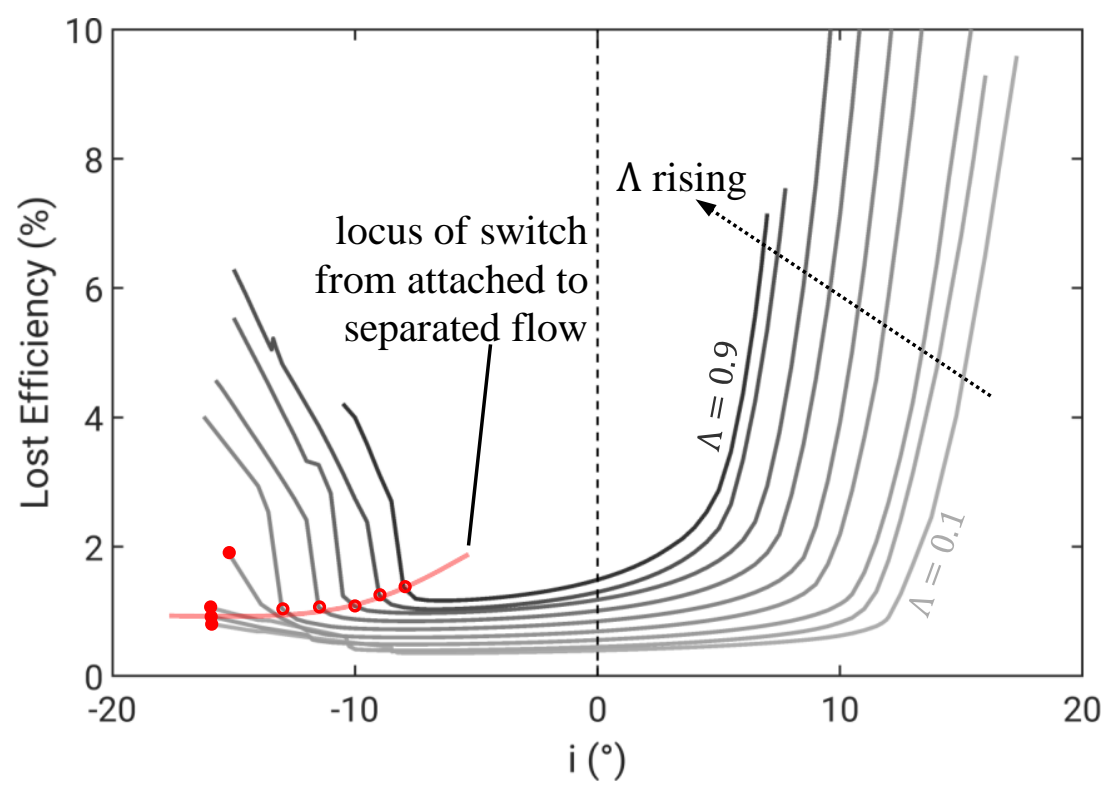

Figure 4.10: Rotor lost efficiency loop showing the effect of reaction on incidence range for the case of solidity set by constant $\mathrm{H}_{\mathrm{te}}$ (MISES). Line increment: 0.1. 
The incidence in Figure 4.10 is given by:

$$
\mathrm{i}^{\circ}=\alpha_{\text {actual }}^{\circ}-\alpha_{\text {design }}^{\circ}
$$

where $\alpha^{\circ}$ actual is the relative inlet angle into the blade row at incidence $i^{\circ}$ and $\alpha^{\circ}$ design is the angle at which 'smooth flow' occurs.

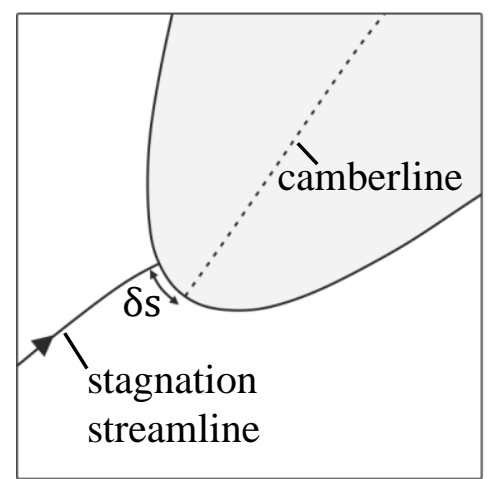

negative incidence

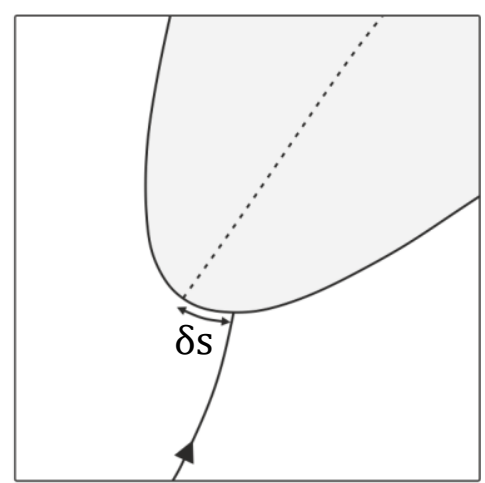

positive incidence

Figure 4.11: Definition of negative incidence (left) and positive incidence (right).

If $\alpha_{\text {actual }}$ drops relative to $\alpha_{\text {design }}$, the stagnation streamline meets the suction-surface as shown in the left-hand side of Figure 4.11. This results in negative incidence according to the definition given by equation 4.10 . If $\alpha_{\text {actual }}$ rises relative to $\alpha_{\text {design }}$, the stagnation streamline meets the pressure-surface as shown in the right-hand side of Figure 4.11. This results in positive incidence.

Figure 4.12 shows how the range of efficient operation of the stator changes with reaction, for the case of constant $\mathrm{H}_{\text {te }}$. It is clear that as reaction drops, setting $\mathrm{H}_{\text {te }}$ results in a narrower range of efficient operation. Figure 4.12 for the stator is identical to Figure 4.10 for the rotor except that the lines of lost efficiency are one minus reaction, $1-\Lambda$.

Figure 4.10 for the rotor and Figure 4.12 for the stator demonstrate that there is an argument for $50 \%$ reaction maximising the operating range, based on balancing the critical level of incidence in the rotor and stator. At any value of reaction the critical level 
is reached in one blade row, before the other. There is therefore a reduction in operating range for asymmetric velocity triangles.

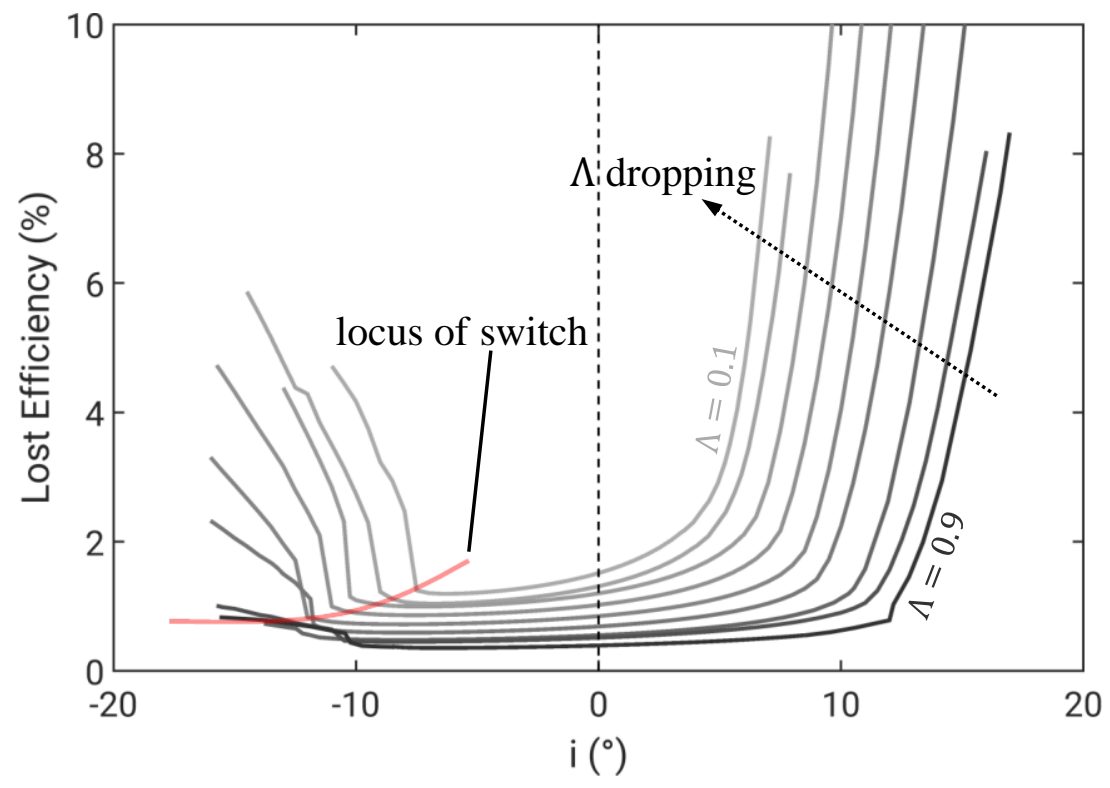

Figure 4.12: Stator lost efficiency loop showing the effect of reaction on incidence range for the case of constant $\mathrm{H}_{\mathrm{te}}$ (MISES). Line increment: 0.1 .

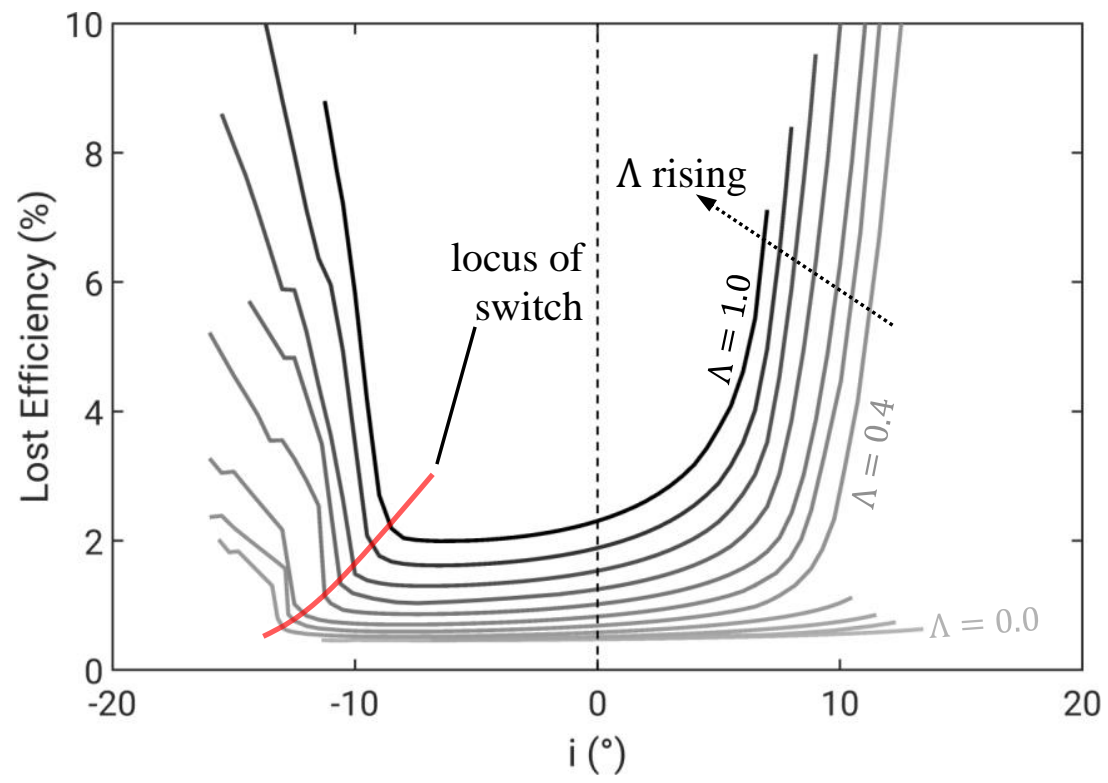

Figure 4.13: Lost efficiency loop showing the effect of reaction on rotor incidence range for the case of constant solidity (MISES). Line increment: 0.1 . 
Figure 4.13 shows the case of constant solidity, for the rotor. Compared to Figure 4.10 for the case of constant $\mathrm{H}_{\text {te }}$, the overall shape is markedly similar, although there is a wider range of efficient operation for low reactions $(\Lambda<0.4)$. This is consistent with Figure 4.9 which shows that the rotor is over-bladed at low reactions. The $70 \%$ reaction compressor has a critical negative incidence of approximately $-12.5^{\circ}$, similar to that in Figure 4.10. However, the locus of the switch from attached to separated flow is significantly steeper for the case of constant $\mathrm{H}_{\mathrm{te}}$.

Figure 4.14 shows the case of constant $\mathrm{D}_{\mathrm{eq}}^{*}$, for the rotor. Compared to Figure 4.10 for the case of constant $\mathrm{H}_{\text {te }}$, it is clear that there is a narrower range of efficient operation for all reactions. This is consistent with Figure 4.9 which shows that the rotor becomes progressively under-bladed as the reaction moves away from $50 \%$. The $70 \%$ reaction compressor has a critical negative incidence of approximately $-9.0^{\circ}$, for the case of constant $\mathrm{D}_{\mathrm{eq}}^{*}$, which is $3.5^{\circ}$ less than that observed for the case of constant $\mathrm{H}_{\mathrm{te}}$. The locus of the switch from attached to separated flow is significantly shallower for the case of constant solidity.

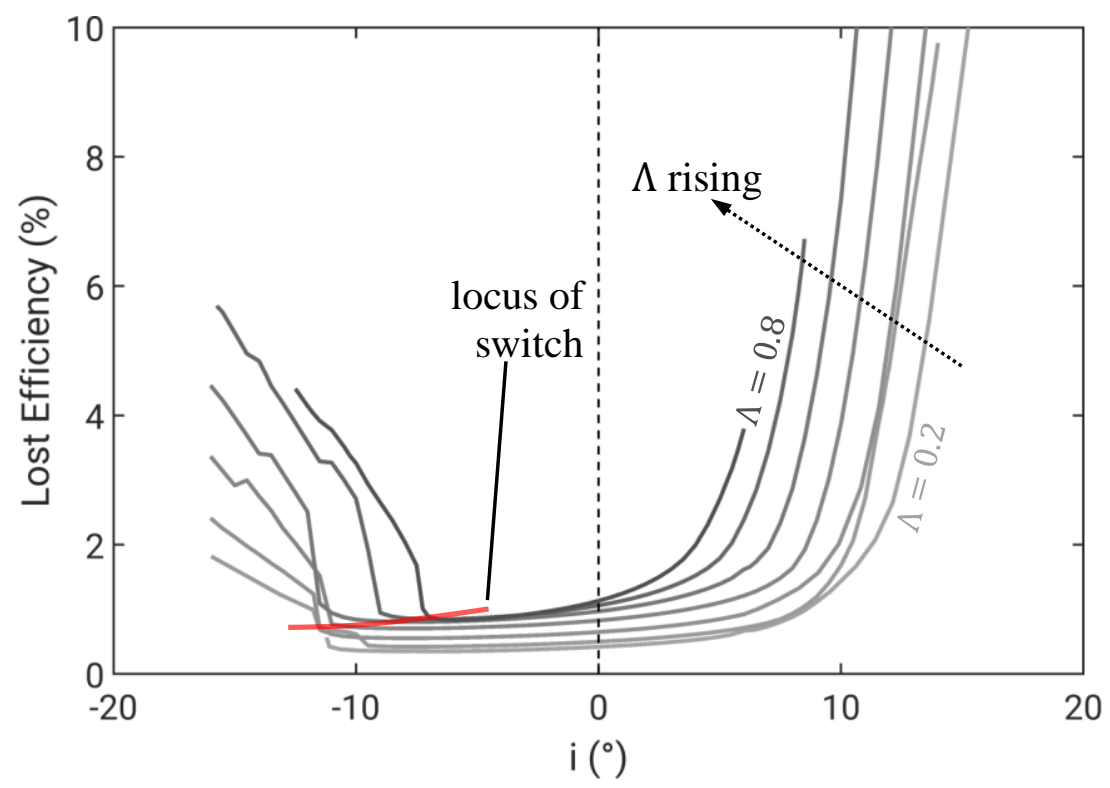

Figure 4.14: Lost efficiency loop showing the effect of reaction on rotor incidence range for the case of constant $\mathrm{D}_{\mathrm{eq}}^{*}$ (MISES). Line increment: 0.1 . 


\subsection{Summary}

The methodology by which the solidity is set is critical in determining the effect of reaction on profile loss. The effect of vary solidity is also critical in determining the effect of reaction on incidence range. To understand this effect, a framework of lost efficiency was developed.

Whether 50\% reaction results in the most, or least, efficient compressor depends on a trade between the effect of solidity (term 1 in equation 4.3) and the effects of changing velocity triangle and blade loss coefficient (term 2 and term 3 in equation 4.3). This trade depends on the particular flow coefficient $\Phi$ and work coefficient $\Psi$ at which the compressor is designed.

Most importantly, when the solidity is set by the suction-surface boundary layer shape factor at the trailing-edge $\mathrm{H}_{\mathrm{te}}$, and conventional levels of flow coefficient $\left(\Phi_{\mathrm{d}}=0.597\right)$ and work coefficient $\left(\Psi_{\mathrm{d}}=0.436\right)$ are used, the profile loss becomes relatively independent of reaction. In fact increasing the reaction from $50 \%$ to $70 \%$ reduces the stage efficiency by only $0.13 \%$.

Finally, setting the solidity constant, or fixing $\mathrm{D}_{\mathrm{eq}}^{*}$ constant, is undesirable. In the case of constant $\mathrm{D}_{\mathrm{eq}}^{*}$, as the reaction moves away from $50 \%$ the boundary layers are driven toward separation. In the case of constant solidity, moving away from 50\% reaction leaves the stage over-bladed. 


\section{Chapter 5}

\section{Endwall with rotation switched off}

In this chapter the effects of reaction on the endwall flow, with the effects of rotation switched off, are presented. The solidity of both the rotor and the stator have been set by fixing the boundary layer shape factor at the trailing-edge of the suction-surface $\mathrm{H}_{\mathrm{te}}$, as described in Section 3.3.3. This chapter uses the CFD setup described in Section 3.1.2. The results presented have been produced using the linear repeating stage model, described in Section 3.4. Conventional levels of design flow coefficient $\left(\Phi_{d}=0.597\right)$ and work coefficient $\left(\Psi_{\mathrm{d}}=0.436\right)$ are fixed throughout this chapter.

The chapter is divided into six parts. In the first part the effect of reaction on the design point efficiency of the stage is studied. Second the effect of reaction on the operating range of the compressor is studied. This raises the question of what controls the maximum pressure rise capability of the stage, which is answered in the third part. Fourth the effect of reaction on the endwall velocity triangle is investigated. Fifth the underlying mechanism is explained. At the end of the chapter, there is a summary.

\subsection{Design loss}

The endwall loss is defined as the total loss minus the profile loss. It follows that the endwall loss can therefore be written as: 


$$
\left(\frac{\mathrm{T} \Delta \mathrm{s}}{\Delta \mathrm{h}_{0}}\right)_{\text {endwall }}=\frac{(\mathrm{T} \Delta \mathrm{s})_{\text {total }}-(\mathrm{T} \Delta \mathrm{s})_{\text {profile }}}{\Delta \mathrm{h}_{0}}
$$

where $(\mathrm{T} \Delta \mathrm{s})_{\text {profile }}$ is the loss associated with the aerofoil profile and $(\mathrm{T} \Delta \mathrm{s})_{\text {total }}$ is the total loss, across the stage. It is clear from the definition of endwall lost efficiency given by equation 5.1, that the endwall loss is the contribution to the total loss of the stage, due to the additional presence of the endwalls.

The effect of reaction on the hub endwall loss is shown in Figure 5.1. The black line shows the case with zero clearance and the red line shows the case with a $1 \%$ stator hub clearance. For clarity, the casing endwall loss has not been plotted. It is identical to the hub endwall loss except that the $\mathrm{x}$ axis is one minus reaction, $1-\Lambda$. The hub endwall loss can be seen to rise as reaction rises.

The cause of the rise can be understood by considering the loss which would occur in a turbulent boundary layer over the hub endwall. The boundary layer edge velocity is considered to vary axially, and to be equal to the circumferentially mass-averaged blade mid-span relative velocity $\mathrm{W}(\mathrm{x})$. The entropy generation rate in such a boundary layer, per unit pitch, is given by:

$$
\dot{S}=\int \frac{C_{d} \rho W^{3}(x)}{T} d x
$$

where the value of the dissipation coefficient, $C_{d}$, in equation 5.2 is set as 0.002 , Denton (1993). Writing equation 5.2 in the form of the hub lost efficiency gives the blue line in Figure 5.1. The exact form of the equation plotted is derived in Appendix A. 


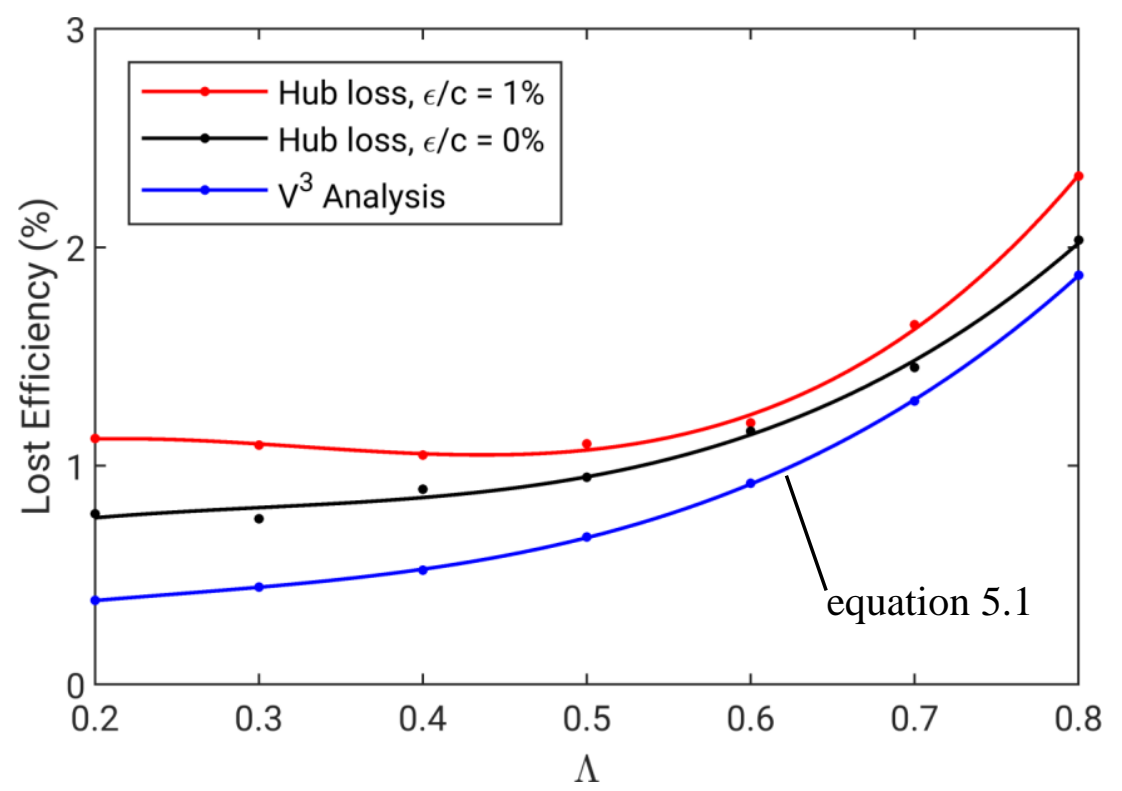

Figure 5.1: Hub endwall lost efficiency (3-D CFD).

A comparison of the blue line and the black line in Figure 5.1 shows that the rise in loss, as the reaction is raised, is simply caused by the rise in the freestream velocity relative to the hub endwall.

The cause of the rise in the freestream velocity relative to the hub endwall can be understood by looking at the velocity triangles in Figure 5.2. As the reaction is raised, the relative velocity into both the rotor and the stator, $W_{1}$ and $W_{2}$, rise. This can be understood more intuitively from Figure 5.3. The figure shows a schematic of the time averaged midheight streamline in the relative frame. As the reaction is raised the rotor stagger rises. Because the rotor is the blade which is connected to the hub endwall, it sets the mean flow angle relative to that endwall. Raising the rotor stagger, for a constant axial velocity, therefore raises the endwall relative velocity $\mathrm{W}_{\text {in }}$ into both the rotor and stator.

To a first order a designer can therefore estimate whether the loss on an endwall is either high or low, simply by looking at the stagger of the blade row which is connected to that endwall. 


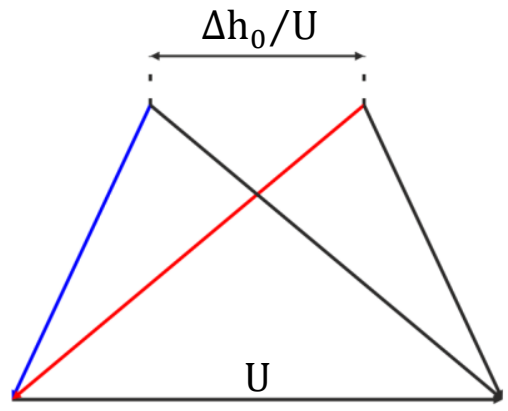

$50 \%$ reaction

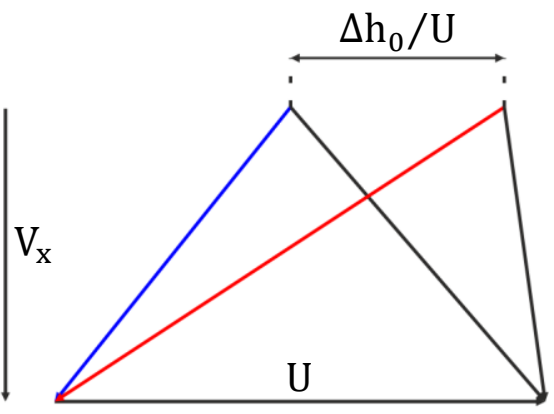

$70 \%$ reaction

Figure 5.2: Effect of reaction on velocity triangle asymmetry. 50\% reaction (left) and $70 \%$ reaction (right).

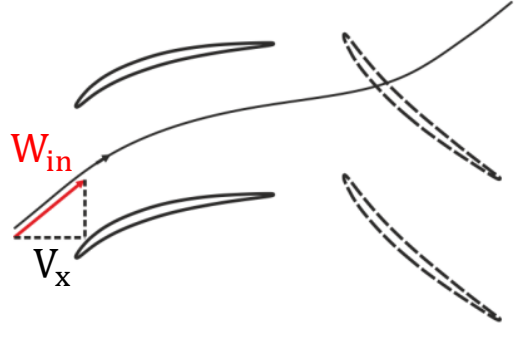

$30 \%$ reaction

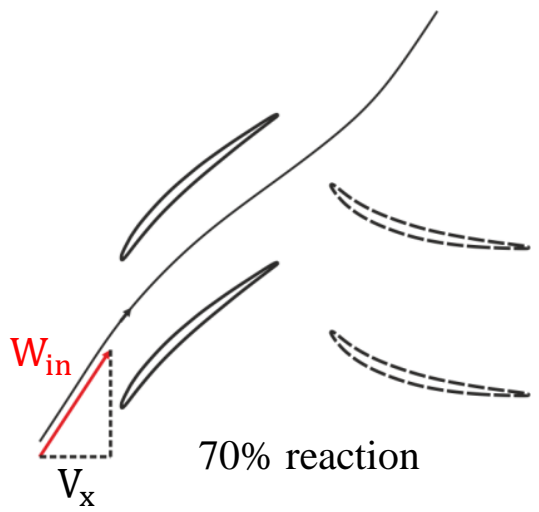

Figure 5.3: Mid-span streamline relative to hub for 30\% reaction (left) and 70\% reaction (right) stages.

A secondary effect of reaction on endwall loss can be seen in Figure 5.1. Comparing the black line, the case with no stator hub clearance, and the red line, the case with a $1 \%$ stator hub clearance, it can be seen that the effect of the hub gap on endwall loss drops as the reaction is raised. On the hub endwall, the clearance gap is on the stator hub and so this shows that as the stagger of the stator is reduced the hub leakage loss drops. An identical behaviour was observed on the casing. In summary, when the stagger of a blade was reduced the leakage loss was found to drop. 
Figure 5.4 shows the effect of reaction on the total lost efficiency of the stage. For both the cases without and with rotor and stator clearances, the $50 \%$ reaction compressor is the most efficient. Table 5.1 summarises the results in Figure 5.4 by comparing the difference in lost efficiency between the $70 \%$ and $50 \%$ reaction stage. As expected from the findings in the previous chapter, fixing solidity by setting the boundary layer shape factor at the trailing-edge of the suction-surface $\mathrm{H}_{\text {te }}$, results in the profile loss of the stage becoming relatively independent of reaction. Table 5.1 shows that changing reaction from $70 \%$ to $50 \%$ reduces the endwall lost efficiency, causing an increase in stage efficiency of $0.49 \%$, for the case with clearances, and $0.58 \%$, for the case without clearances. For both the cases without and with rotor and stator clearances, approximately four fifths of this increase in stage efficiency arises from the reduction in endwall lost efficiency.

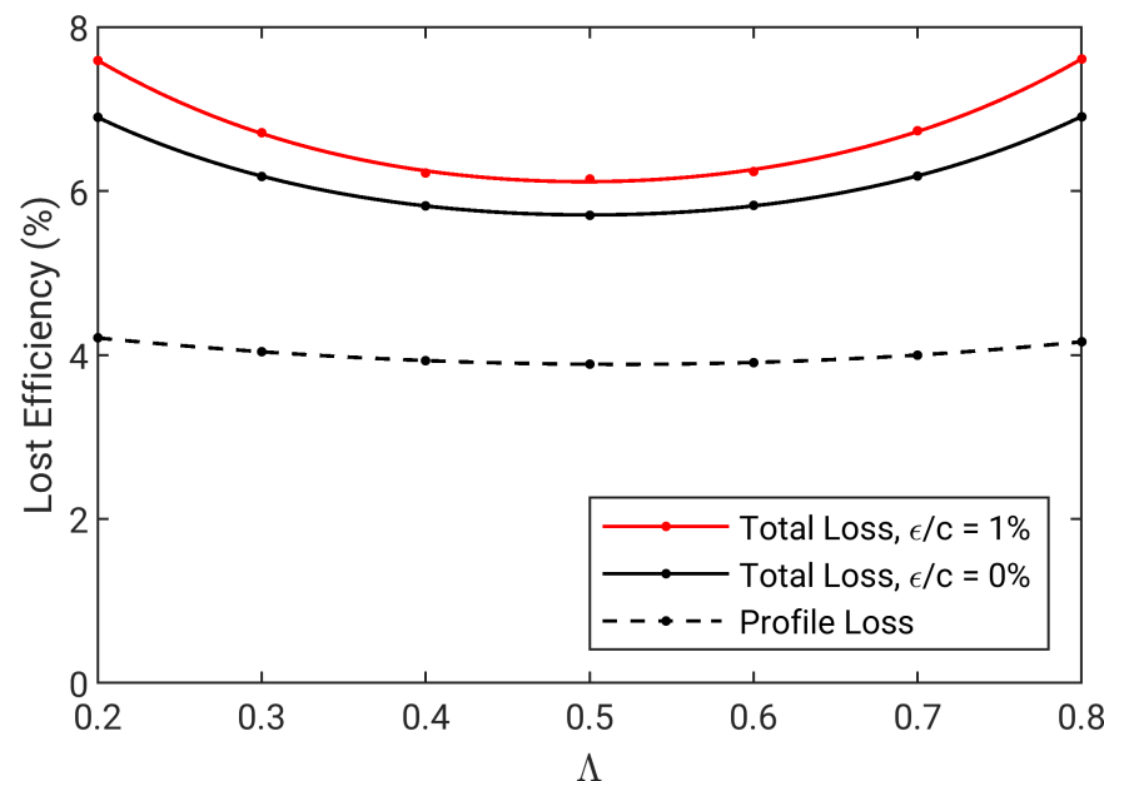

Figure 5.4: Variation of lost efficiency with reaction (3-D CFD).

Table 5.1: Summary of the effect of reaction on design efficiency (3-D CFD).

\begin{tabular}{|l|r|r|}
\hline \multicolumn{1}{|c|}{$\eta_{\Lambda=70 \%}-\eta_{\Lambda=50 \%}(\%)$} & $0 \%$ clearances & $1 \%$ clearances \\
\hline Endwall & -0.47 & -0.38 \\
\hline Profile & -0.11 & -0.11 \\
\hline Total & -0.58 & -0.49 \\
\hline
\end{tabular}




\subsection{Operating range}

The effect of reaction on the operating range of the compressor, for the cases without clearances and with clearances, are shown in Figures 5.5 and 5.6. Here we define the static-pressure rise coefficient of the compressor as:

$$
\psi^{\prime}=\frac{\Delta p}{\frac{1}{2} \rho U^{2}}
$$

where $\Delta \mathrm{p}$ is the static-pressure rise across the stage and $\mathrm{U}$ is the absolute rotor blade speed. On each plot, the dotted line shows the maximum pressure rise throttle characteristic at the point at where the CFD solution started to diverge. For both cases without clearances and with clearances, the operating range of the compressor can be seen to drop as the reaction is moved away from 50\%. This effect is small. Increasing the reaction from $50 \%$ to $70 \%$ reduces the minimum flow coefficient $\Phi_{\min }$ from 0.442 to 0.447 , for the case without clearances. This is a reduction of $1.1 \%$, relative to the $50 \%$ reaction case. For the case with clearances, the $\Phi_{\min }$ drops by $2.9 \%$.

To compare the maximum pressure rise between compressors, the maximum pressure rise throttle coefficient, $\mathrm{k}$, is used. This is a measure of the exit area at maximum pressure rise and is defined by:

$$
\mathrm{k}=\frac{\Psi_{\max }^{\prime}}{\Phi_{\min }^{2}}
$$

where $\psi_{\max }^{\prime}$ is the maximum static-pressure rise coefficient and $\Phi_{\min }$ is the flow coefficient at which $\psi_{\max }^{\prime}$ occurs. The use of $\mathrm{k}$ is well-documented and validated, and has been used extensively to compare the operating range between compressors, for example by Taylor et al. (2020) to study the operability of damaged compressors. 


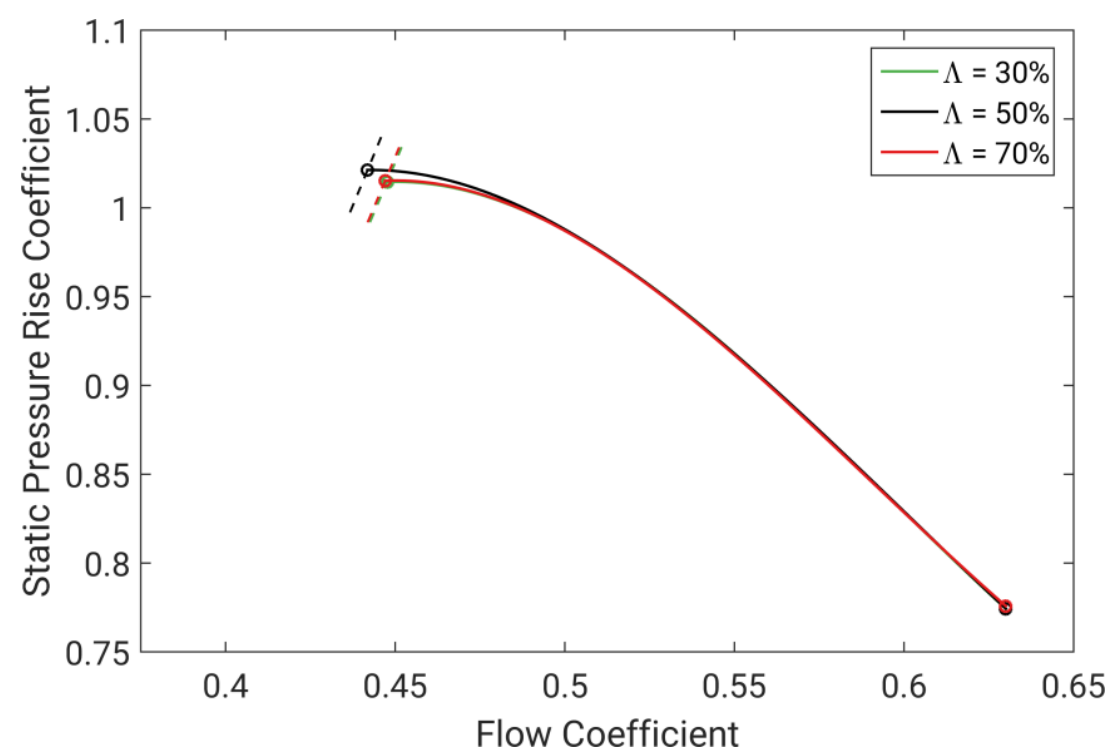

Figure 5.5: Static-pressure rise characteristics for compressors without clearances (3-D CFD).

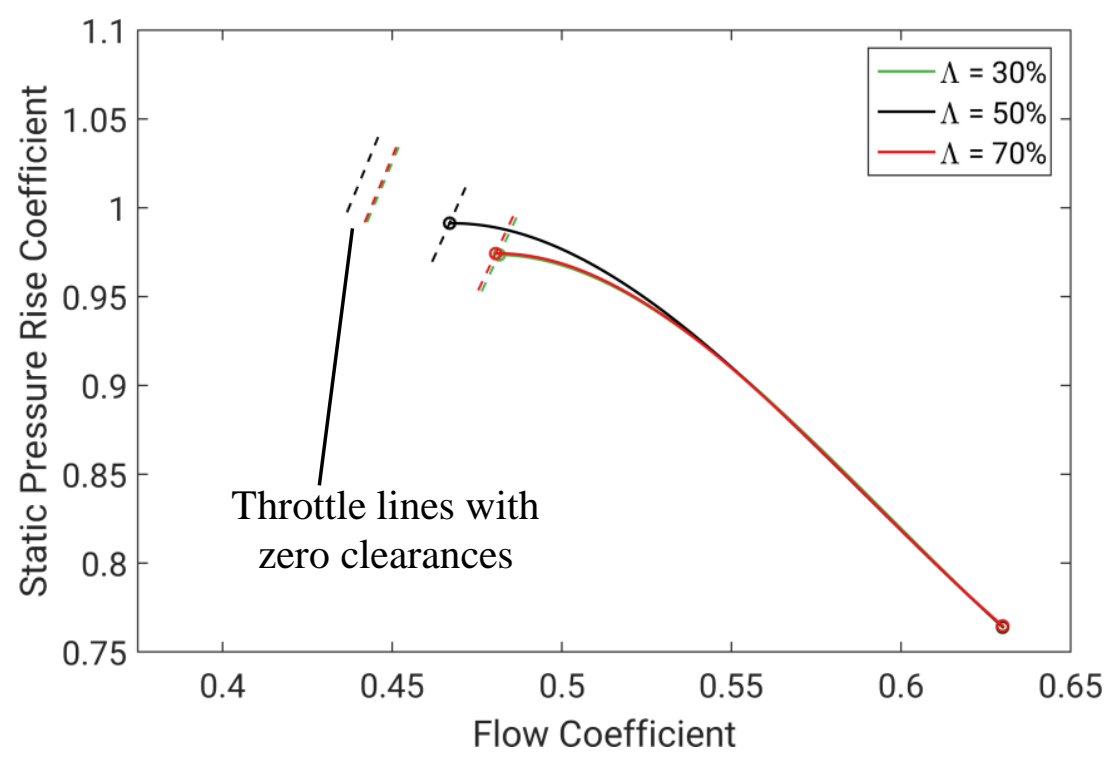

Figure 5.6: Static-pressure rise characteristics for compressors with clearances (3-D CFD).

Figure 5.7 gives a summary of how the maximum pressure rise throttle coefficient $\mathrm{k}$ varies with reaction. For both cases, without clearances and with clearances, the $50 \%$ reaction compressor has the largest operating range. It can be seen that the variation in operating range is symmetrical about $50 \%$ reaction. Increasing reaction from $50 \%$ to $70 \%$ reduces 
the maximum pressure rise throttle coefficient $\mathrm{k}$ from 5.36 to 4.85 , for the case without clearances. This is a reduction of $9.5 \%$, relative to the $50 \%$ reaction case. For the case with clearances, $\mathrm{k}$ drops by $7.2 \%$.

It can be seen that the addition of clearances reduces the operating range at all reactions, by a similar amount. The difference in $\mathrm{k}$ between the cases without clearances and with clearances is approximately 0.72 .

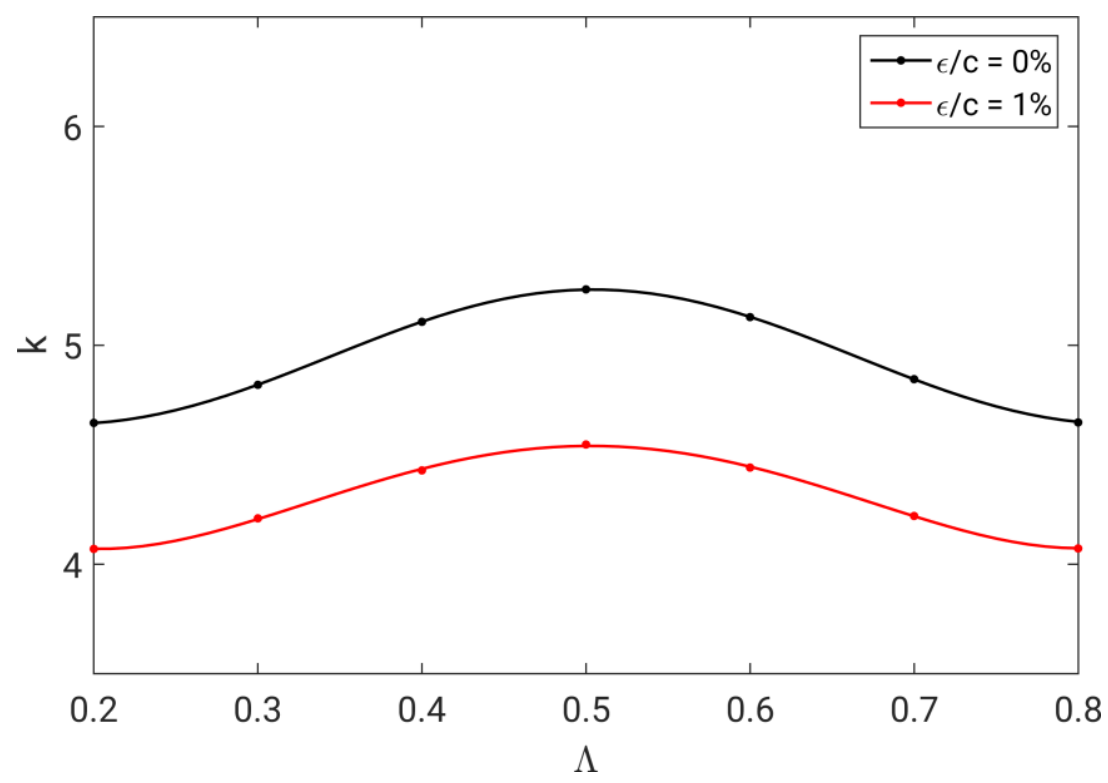

Figure 5.7: Maximum pressure rise throttle coefficient (3-D CFD).

\subsection{What controls maximum pressure rise?}

The cause of the reduction in the maximum pressure rise throttle coefficient $\mathrm{k}$ can be seen in Figure 5.8. The figure shows the limiting surface streamlines at close to maximum pressure rise $(\Phi=0.461)$ for the stator of the $50 \%$ and $70 \%$ reaction compressor stages with zero clearance. This shows the surprising result that the reduction in maximum pressure rise, as reaction is raised, is the result of an increase in the size of the stator hub separation. This is unexpected because as reaction rises, the static-pressure rise across the rotor increases and the static-pressure rise across the stator decreases. 
The opposite occurs, but in the rotor casing, as the reaction is dropped from 50\% to $30 \%$. In this case the reduction in maximum pressure rise occurs because the size of the separation in the rotor casing increases. This case is not shown for brevity.

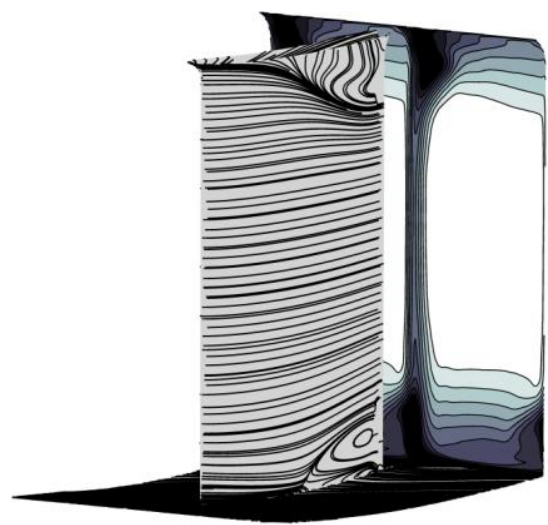

$50 \%$ reaction

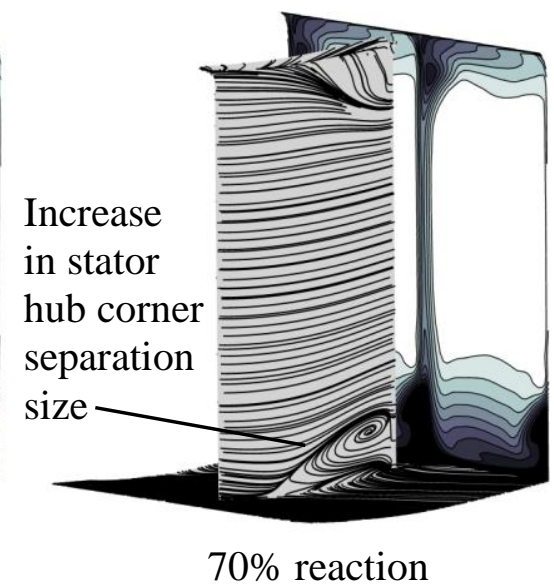

Figure 5.8: Stator suction-surface limiting streamlines for the case of zero clearances (3D CFD).

The cause of the increase in the size of the stator hub corner separation at high reaction can be understood by looking at the spanwise distribution of the local static-pressure rise coefficient across the stator, shown in Figure 5.9. Here we follow Auchoybur and Miller (2017) and define the local static-pressure rise coefficient $C_{p}$ as:

$$
\mathrm{C}_{\mathrm{p}}=\frac{\Delta \mathrm{p}}{\frac{1}{2} \rho \mathrm{V}_{\text {local }}^{2}}
$$

where $\Delta \mathrm{p}$ is the static-pressure rise across the stator and $\mathrm{V}_{\text {local }}$ is the relative inlet velocity into the stator. The bottom of equation 5.5 is the inlet dynamic pressure into the stator at each span fraction. Figure 5.9 shows that as the reaction is raised the local static-pressure rise coefficient in the stator hub rises. This rise in the local static-pressure rise coefficient in the stator hub causes the rise in the size of the stator hub separation, seen in Figure 5.8. 


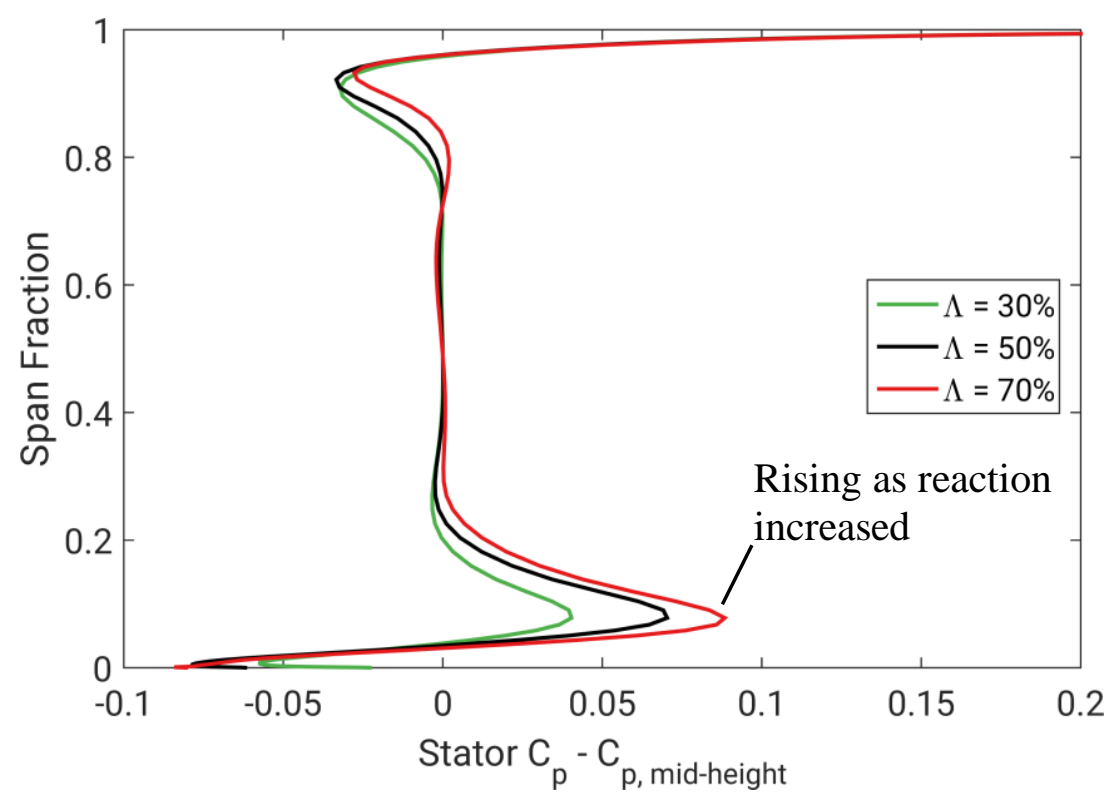

Figure 5.9: Spanwise variation in stator static-pressure rise coefficient with zero clearance (3-D CFD).

\subsection{Effect of reaction on endwall velocity triangle}

The rise in local static-pressure rise coefficient in the stator hub is caused by a drop in stator inlet velocity, $\mathrm{V}_{\text {local }}$ in equation 5.5, in the stator hub endwall region. The cause of this drop can be understood by looking at the stator inlet velocity triangle. This shows the re-energising effect, caused by the change in frame of reference, described by Koch (1981) and Auchoybur and Miller (2017) and discussed in Section 2.1.5.

Figure 5.10 shows the freestream and hub endwall stator inlet velocity triangles for $30 \%$ and $70 \%$ reaction. The freestream and hub endwall velocities have been extracted from the CFD by mass-averaging the velocities over $25 \%$ to $75 \%$ of the mass flux and $0 \%$ to $25 \%$ of the mass flux respectively. This is illustrated schematically in Figure 5.11. Returning to Figure 5.10 shows that as the reaction rises from $30 \%$ to $70 \%$ the relative difference between $V_{f s}^{2}$ and $V_{\text {hub }}^{2}$ rises. This is caused by two effects. First, as reaction is raised, the axial velocity $V_{x}$ in the hub region drops. Second, as the reaction rises the magnitude of freestream stator inlet velocity $V_{\text {fs }}$ drops and therefore any drop in $V_{\text {hub }}$ 
causes a larger fractional change in the dynamic pressure entering the stator in the hub endwall region.

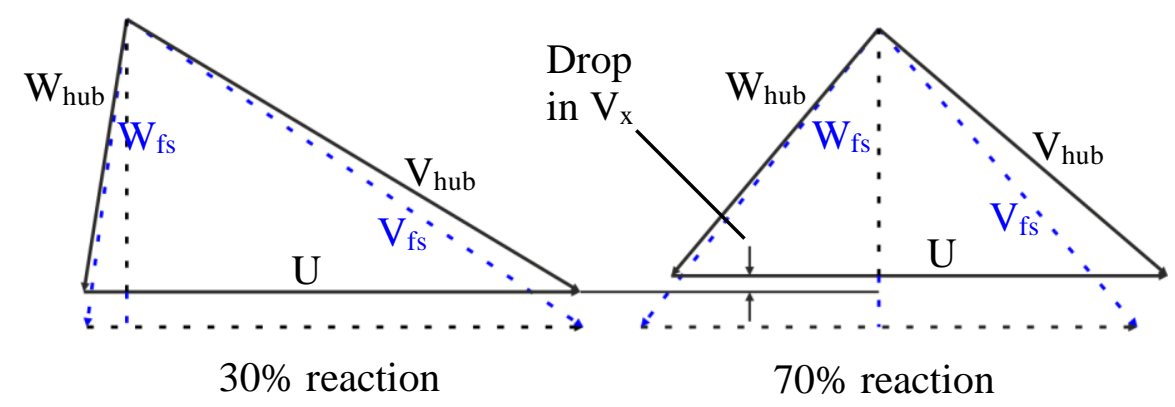

Figure 5.10: Stator inlet velocity tringles for hub endwall (solid) and freestream (dashed) regions.

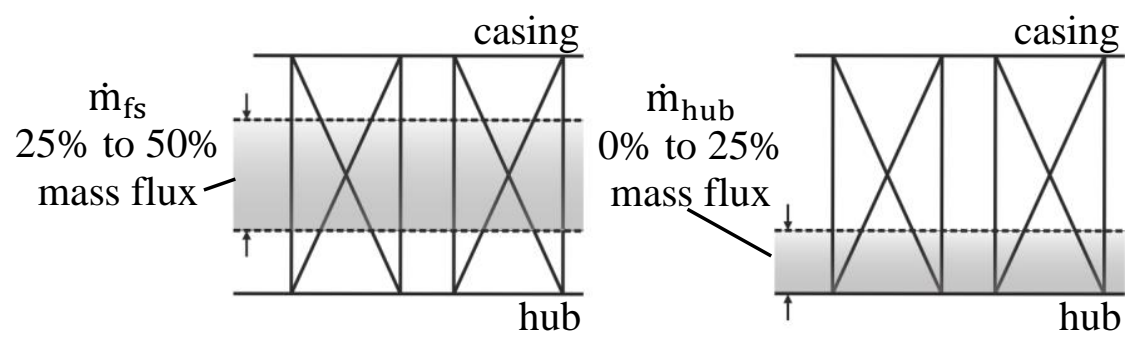

Figure 5.11: Decomposition of the flow into freestream (left) and hub endwall (right).

\subsection{Underlying mechanism}

Finally, it is necessary to explain why the axial velocity in the hub endwall region drops as the reaction is raised. Consider once again the hub streamtube ( $0 \%$ to $25 \%$ of the mass flux) used to create Figure 5.10 and shown schematically on the right-hand side of Figure 5.11. Now the mass-averaged change in stagnation enthalpy, stagnation pressure and the entropy across the stage is extracted from the CFD. As the flow is incompressible the three are related by the fundamental thermodynamic relation: 


$$
\frac{\Delta \mathrm{h}_{0}}{\mathrm{U}^{2}}=\frac{\Delta \mathrm{p}_{0}}{\rho \mathrm{U}^{2}}+\frac{\mathrm{T}_{0} \Delta \mathrm{s}}{\mathrm{U}^{2}}
$$

In the endwall region there are two restrictions on equation 5.6. First, the second term, the stagnation pressure rise coefficient, must be constant as reaction is changed, shown in the left-hand side of Figure 5.12. This is because in a repeating stage the stagnation pressure rise coefficient is constant across the span and all stages have been designed to achieve the same stagnation pressure rise coefficient. Second, the first term in equation 5.6, the work coefficient, must always collapse onto the same characteristic, shown in the right-hand side of Figure 5.12. This is because in the endwall region the deviation is found to be small, approximately $1^{\circ}$, and is found to be relatively independent of reaction. This means that the work coefficient of the endwall region must collapse onto the same characteristic set by the blade metal angles. It should be noted that as the reaction is changed the gradient of the work coefficient verses flow coefficient characteristic does not change.

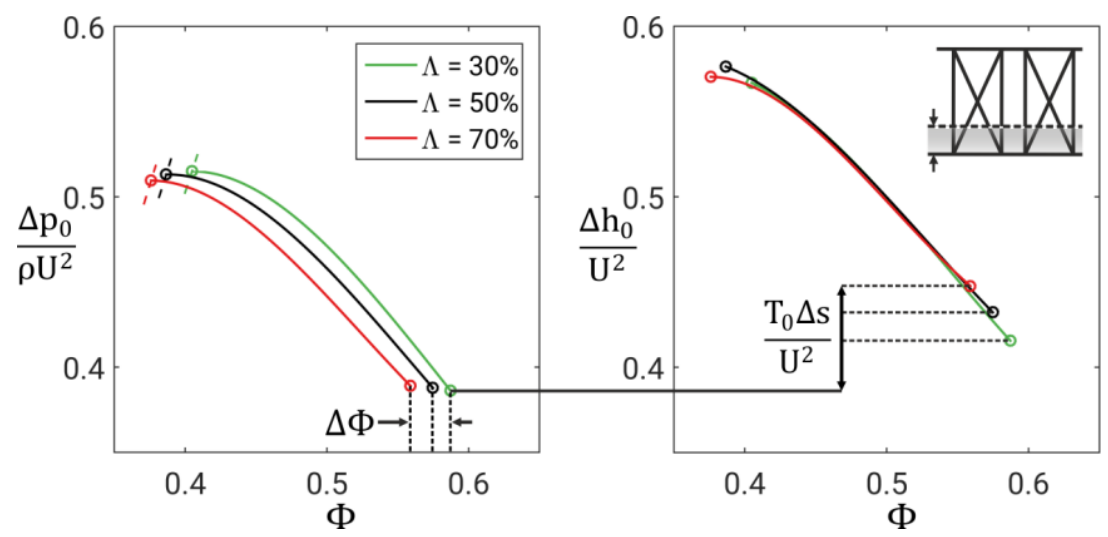

Figure 5.12: Total-pressure rise (left) and work coefficient (right) characteristics for hub endwall region.

These restrictions on the work and stagnation pressure rise coefficient, caused by equation 5.6, are shown graphically in Figure 5.12. They result in the magnitude of the endwall loss, the third term in equation 5.6, fixing the endwall flow coefficient. This effect was also observed by Auchoybur and Miller (2017). As discussed in Section 5.1, as reaction rises the hub endwall loss rises and this causes the mass flow in the endwall to drop. 
To summarise, the reduction in the maximum pressure rise of a stage, as reaction rises, is caused by an increase in the size of the stator hub separation. This is caused by a rise in the local static-pressure rise coefficient of the stator hub. In turn this is caused by a drop in the axial velocity in the stator endwall region as the stator endwall loss rises. A simple schematic is shown in Figure 5.13 which describes this mechanism. The underlying mechanism is founded on the relationship between the endwall loss and the static-pressure rise coefficient in the endwall. In the repeating stage, a rise in endwall loss causes $C_{p}$ in the endwall to rise.

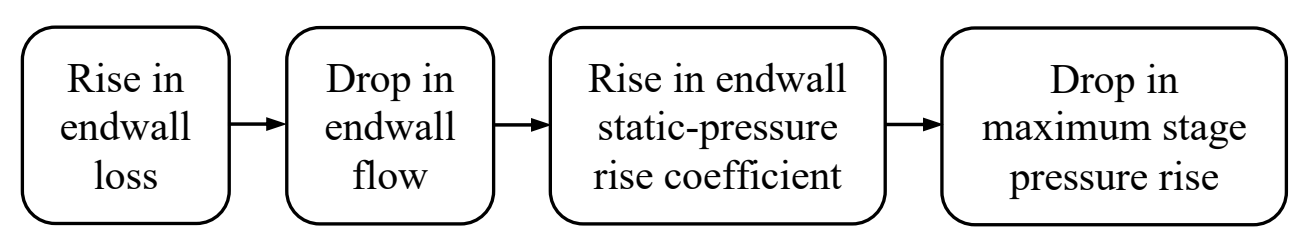

Figure 5.13: Mechanism of maximum pressure rise reduction.

This behaviour is fundamental to all cantilever compressors and shows that as reaction is raised, the stator hub will limit the maximum pressure rise of the stage, and as reaction is dropped the rotor casing will limit the maximum pressure rise of the stage.

\subsection{Summary}

For both the cases without and with rotor and stator clearances, the $50 \%$ reaction compressor was found to be the most efficient. This was understood by looking at the hub endwall loss. The hub endwall loss was shown to rise as reaction rises. The rise in loss, as the reaction is raised, is simply caused by the rise in the freestream velocity relative to the hub endwall. Because the rotor is the blade which is connected to the hub endwall, it controls the mean flow angle relative to that endwall. The rotor stagger therefore approximately sets the mean freestream velocity relative to the hub. A designer can therefore estimate whether the loss on an endwall is either high or low, simply by looking at the stagger of the blade row which is connected to that endwall. 
The operating range of the compressor was observed to drop as the reaction was moved away from $50 \%$, for both the cases without and with rotor and stator clearances. The variation in operating range was symmetrical about $50 \%$ reaction. This is because at $50 \%$ reaction the local static-pressure rise coefficient across each blade endwall is balanced.

The maximum pressure rise capability of the stage was shown to surprising be limited by the blade row with the lowest static-pressure rise, $\Delta \mathrm{p}$. At high reaction, the size of the stator hub corner separation was shown to increase, due to a rise in the local staticpressure rise coefficient across the stator hub. The reason for this was understood by considering the connection between the local static-pressure rise coefficient and the endwall velocity triangle. This showed that as the reaction is increased, there is a rise in hub endwall loss, which in turn causes a drop in axial velocity in the endwall. This reduces the inlet dynamic pressure entering the stator hub causing the static-pressure rise coefficient to increase. Therefore, as the reaction is raised, the stator hub will limit the maximum pressure rise of the stage, and as reaction is dropped the rotor casing will limit the maximum pressure rise of the stage. 


\section{Chapter 6}

\section{Endwall with rotation switched on}

In this chapter the differential rotational effects are reintroduced. This allows the natural asymmetry between the rotor and stator to be examined. This Chapter uses the CFD setup described in Section 3.1.2. The results presented have been produced using the rotation model, described in Section 3.5. Conventional levels of design flow coefficient $\left(\Phi_{d}=0.597\right)$ and work coefficient $\left(\Psi_{d}=0.436\right)$ are fixed throughout this chapter. The centrifugal forces equivalent to a real compressor of hub-to-tip ratio equal to 0.8 have been introduced throughout this chapter, except in Section 6.6 where the centrifugal forces are varied.

The chapter is divided into seven parts. In the first part the effect of rotation on the design loss of the stage is studied. Second the effect of rotation on the operating range of the compressor is studied. This raises three questions. Why does rotation extend the operating range of the compressor? Why are rotation effects larger in the rotor than in the stator? Why does rotation extend operating range further at high reaction? Each question is answered in turn, in parts three, four and five. Sixth the effect of varying the level of rotation is examined. At the end of the chapter, there is a summary. 


\subsection{Design loss}

The effect of rotation on the total lost efficiency of the stage is shown in Figure 6.1. It can be seen that rotation has a relatively small effect on design loss. However, the reaction which achieves the optimal design efficiency increases by around 5\% reaction (from 50\% reaction to 55\% reaction). It is also important to note that the range of reactions over which the efficiency only varies by $0.01 \%$ is relatively wide, between $50 \%$ reaction and $60 \%$ reaction.

Table 6.1 summarises the results in Figure 6.1 by comparing the difference in lost efficiency between the cases with rotation switched on, $\eta_{\text {rot,ON }}$, and off, $\eta_{\text {rot,ofF }}$. For the case of $70 \%$ reaction, switching on rotation reduces the endwall lost efficiency, causing an increase in stage efficiency of $0.13 \%$, for the case without clearances. Whereas for the $30 \%$ reaction, the endwall lost efficiency increases, causing a decrease in stage efficiency of $0.14 \%$, for the case without clearances. A similar effect can be seen in the cases with clearances. Rotation is found to have a negligible effect on profile loss. It remains almost invariant with reaction.

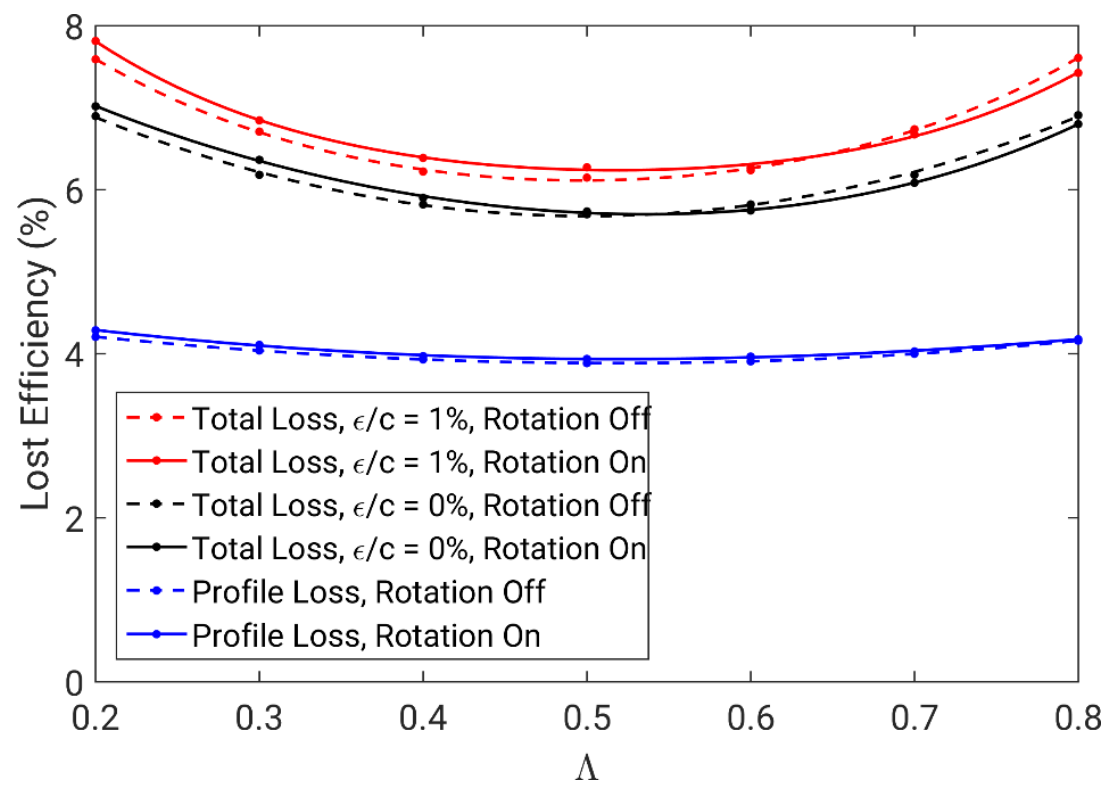

Figure 6.1: Change in lost efficiency for cases with rotation switched on and off (3-D CFD). 
Table 6.1: Summary of the effect of rotation on design efficiency (3-D CFD).

\begin{tabular}{|l|r|r|}
\hline \multicolumn{1}{|c|}{$\eta_{\text {rot, ON }}-\eta_{\text {rot, OFF }}(\%)$} & $0 \%$ clearances & $1 \%$ clearances \\
\hline$\Lambda=30 \%$ & -0.14 & -0.15 \\
\hline$\Lambda=50 \%$ & +0.01 & +0.16 \\
\hline$\Lambda=70 \%$ & +0.13 & +0.07 \\
\hline
\end{tabular}

\subsection{Operating range}

The effects of rotation on the operating range of a compressor are much larger than the effect on design loss. Figures 6.2 and 6.3 show the effect of switching on rotation on compressors with and without clearance. The effect of rotation on the maximum pressure rise throttle coefficient is shown in Figures 6.4 and 6.5. The effect of rotation is to increase the reaction which achieves the maximum pressure rise, by around 15\% (from 50\% reaction to $65 \%$ reaction).

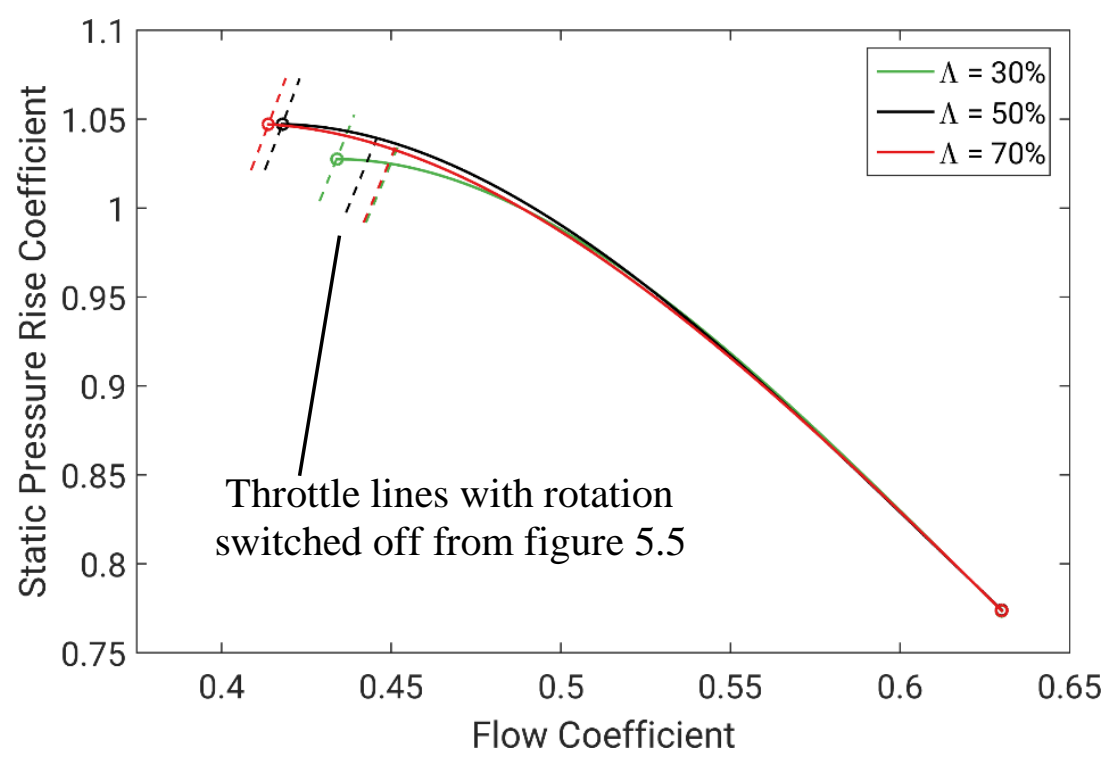

Figure 6.2: Static-pressure rise characteristics for compressors without clearances and rotation on (3-D CFD). 


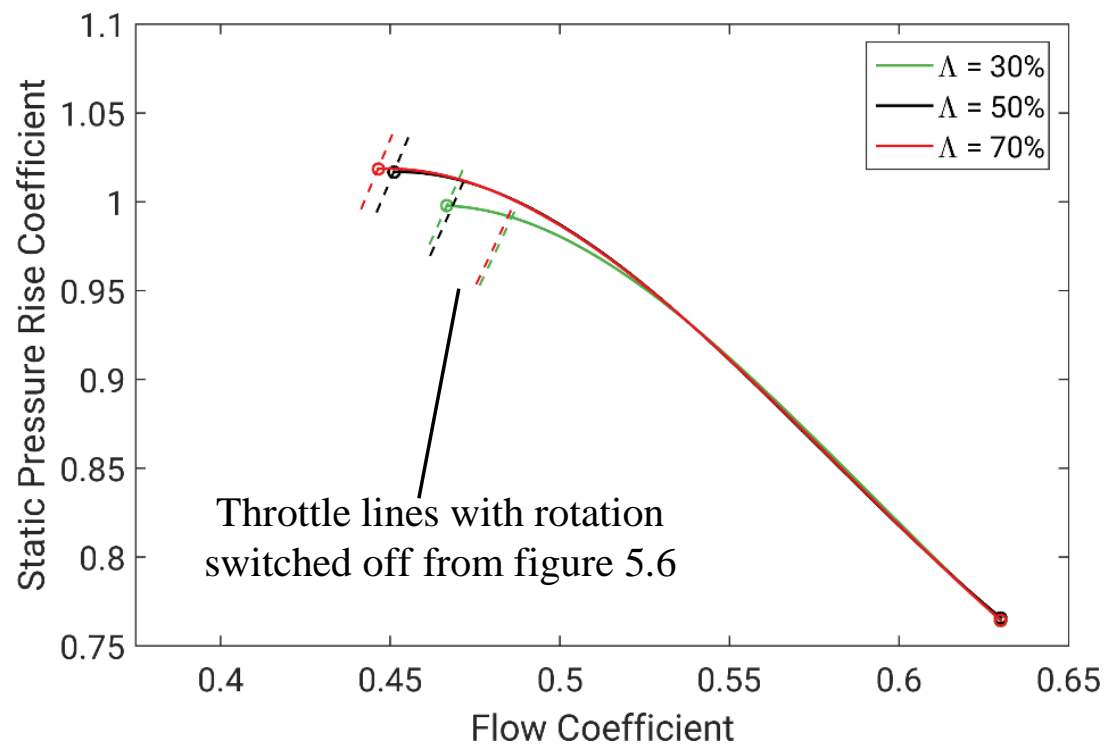

Figure 6.3: Static-pressure rise characteristics for compressors with clearances and rotation on (3-D CFD).

From Figures 6.2 and 6.3, the operating range of all compressors can be seen to rise as rotation is switched on. However, the increase in operating range greatest at high reaction and the variation is no longer symmetrical about $50 \%$ reaction.

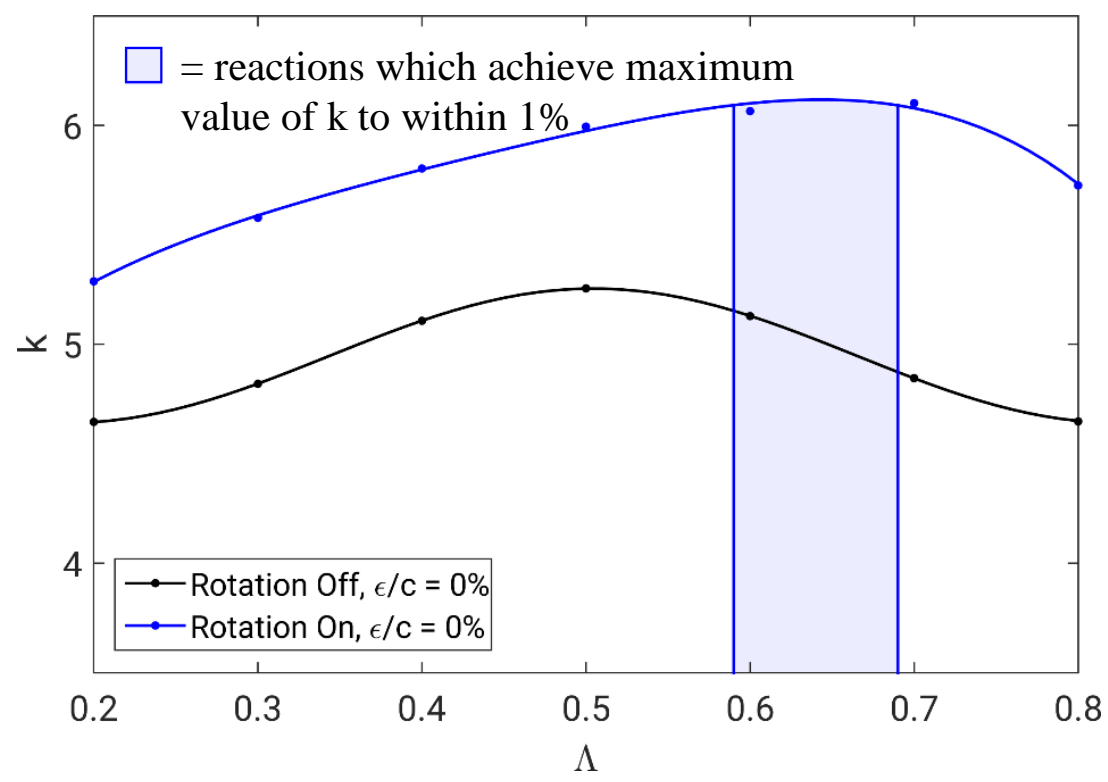

Figure 6.4: Throttle coefficients for cases with zero clearances and rotation switched on and off (3-D CFD). 


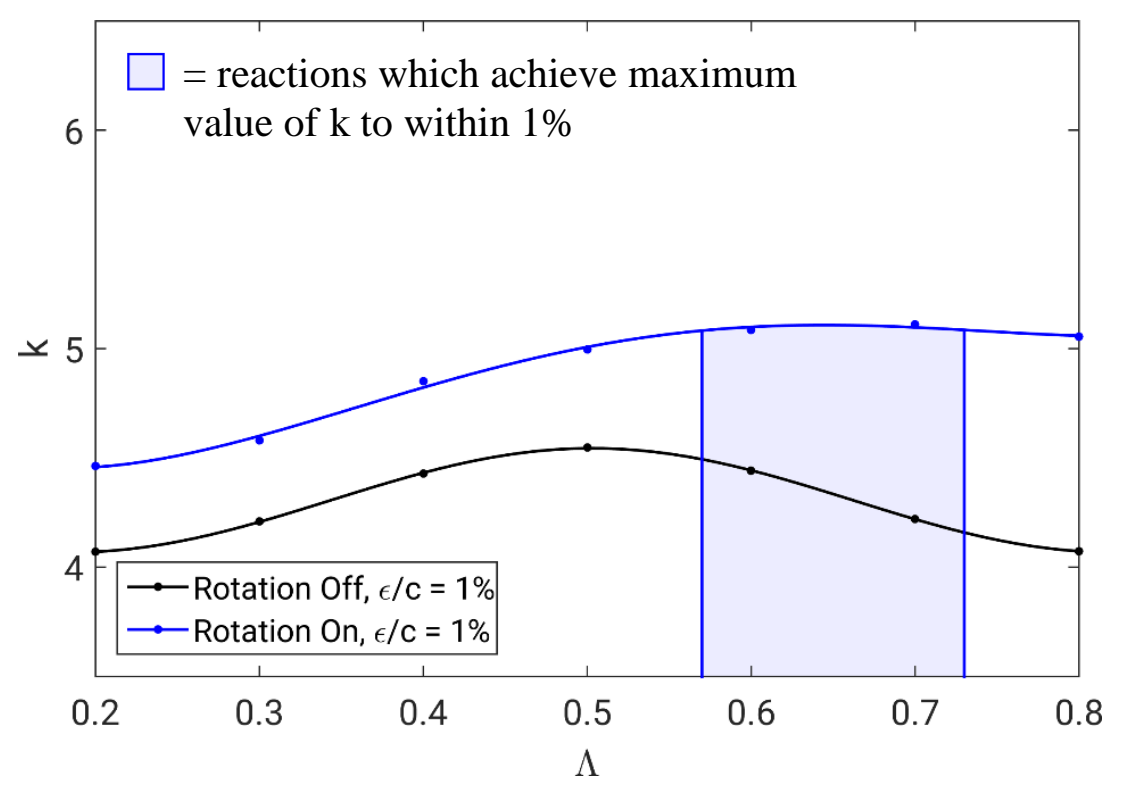

Figure 6.5: Throttle coefficients for cases with $1 \%$ clearances and rotation switched on and off (3-D CFD).

For the case of the 50\% reaction compressor shown in Figure 6.2, switching on rotation increases the minimum flow coefficient $\Phi_{\min }$ by $10.5 \%$ relative to the case with rotation switched off. With rotation switched on the operating range of the $30 \%$ compressor drops by $3.9 \%$ relative to the $50 \%$ reaction compressor. Whereas for the case of the $70 \%$ reaction compressor, there is a relative rise in operating range of $0.1 \%$. Similar results are observed for the cases with clearances. The same effect is observed in Figures 6.4 and 6.5 .

There are two principal effects of rotation on the operating range of the compressor. The first is to the increase the operating range of all compressors. The second is to increase the reaction which achieves the maximum pressure rise, by around $15 \%$ (from $50 \%$ reaction to $65 \%$ reaction). This raises two questions. First, why does rotation extend the operating range of the compressor? Second, why is the extension in operating range larger at high reaction? Each question is answered in turn, in the following sections. 


\subsection{Why does rotation extend operating range?}

The first question, why does rotation extend the operating range of the compressor, can be answered by looking at the 3-D flowfield in the stage. The effect of switching on rotation on the suction-surface limiting streamlines is shown in Figures 6.6 and 6.7. The figures show the compressor at a flow coefficient which is just before maximum pressure rise $(\Phi=0.449)$.

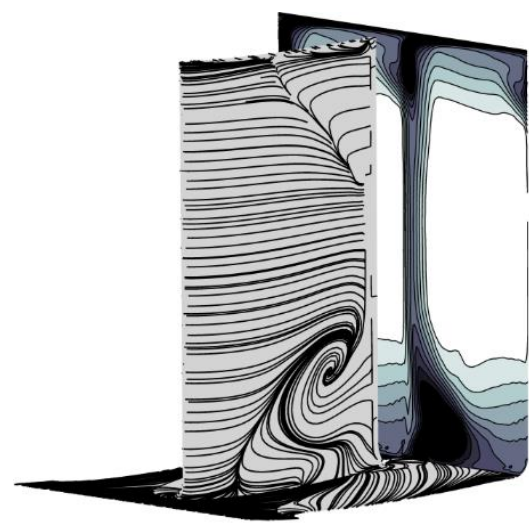

Rotor

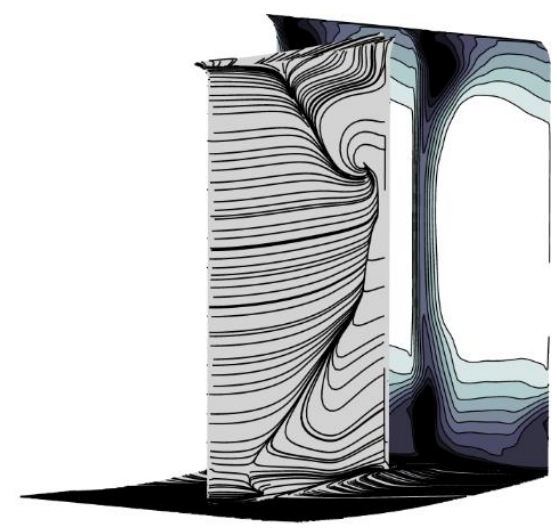

Stator

Figure 6.6: Suction-surface limiting streamlines and entropy loss contours for $50 \%$ reaction with rotation switched off (3-D CFD).

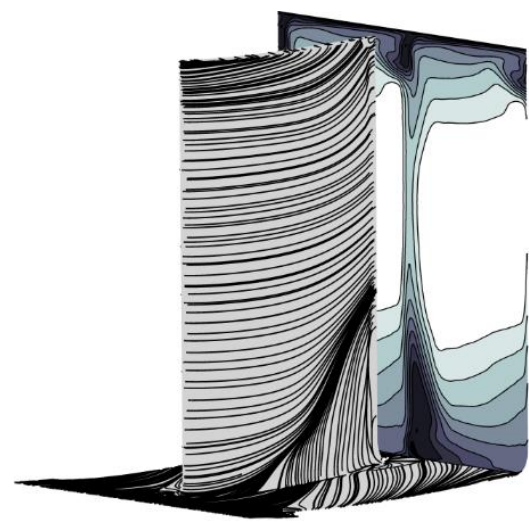

Rotor

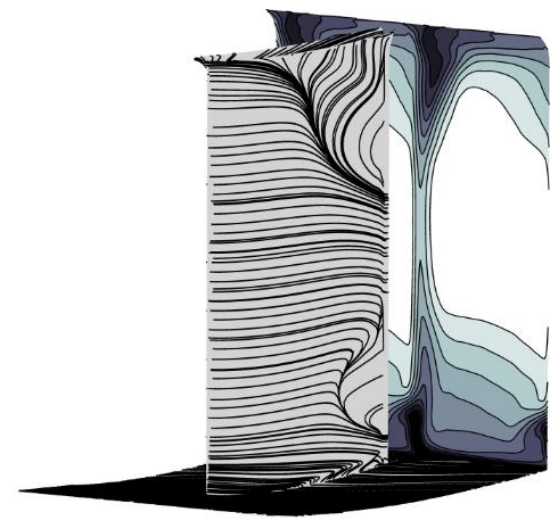

Stator

Figure 6.7: Suction-surface limiting streamlines and entropy loss contours for 50\% reaction with rotation switched on (3-D CFD). 
With rotation switched off, it is clear from the left-hand side of Figure 6.6 that the rotor hub and casing corner separations extend towards the midspan and the blade separates across its span at the trailing-edge. When rotation is switched on, the left-hand side of Figure 6.7 shows that the separation structure in the rotor improves dramatically. The low momentum fluid within the attached boundary layer and the hub corner separation is pumped radially. This provides the boundary layer with a 3-D 'escape mechanism', as the boundary layer is no longer limited to remain in plane. This effect removes both the hub and casing separation allowing the rotor boundary layer to remain attached until the trailing-edge. A similar effect was observed in the stator, by Taylor (2016), as the transverse pressure gradient was increased.

With rotation switched off, it is clear from the right-hand side of Figure 6.6 that the separation structure in the stator is similar to that in the rotor, except that the hub and casing are interchanged. With rotation switched on, it is clear from the right-hand side of Figure 6.7 that the principal effect of switching on rotation, in the stator, is to reduce the size of the stator hub separation. Towards the trailing-edge the boundary layer migrates slightly inwards, towards the hub, but this effect is small.

Figures 6.6 and 6.7 show that with rotation switched off, the maximum pressure rise of the $50 \%$ reaction stage is limited by endwall separations in both the rotor and stator. With rotation switched on however, the mechanism which limits the maximum pressure rise of the stage is different. In this case the maximum pressure rise of the $50 \%$ reaction stage is limited by the casing corner separation in the stator.

The figures show that the effects of rotation are much larger in the rotor than in the stator. Understanding why the effects of rotation are much larger in the rotor, than in the stator is a critical part in understanding both why rotation extends the maximum pressure rise of the compressor, and why the extension in operating range is larger at high reaction. 


\subsection{Why are rotation effects larger in the rotor than in the stator?}

To understand why the effects of rotation are much larger in the rotor, than in the stator, it is necessary to understand how, and where, rotation introduces perturbation centrifugal forces into the rotor and stator boundary layer. To do this a dimensionless parameter is defined, the dimensionless perturbation centrifugal force $F_{c}$, which is a measure of relative magnitude of the perturbation centrifugal forces in the boundary layer.

The dimensionless perturbation centrifugal force $F_{c}$ is defined as the difference between the centrifugal force per unit volume on the blade surface and in the freestream, $\rho \Delta V_{\theta}^{2} / r$, non-dimensionalised by the blade speed squared $U^{2}$, density $\rho$ and the blade span $\Delta \mathrm{r}$. This gives the dimensionless perturbation centrifugal force $\mathrm{F}_{\mathrm{c}}$ as:

$$
\mathrm{F}_{\mathrm{c}}=\frac{\rho\left(\frac{\mathrm{V}_{\theta}^{2}}{\mathrm{r}}\right)_{\text {surface }}-\rho\left(\frac{\mathrm{V}_{\theta}^{2}}{\mathrm{r}}\right)_{\mathrm{fs}}}{\rho \mathrm{U}^{2} / \Delta \mathrm{r}}=\frac{\Delta \mathrm{r}}{\mathrm{r}}\left(\frac{\Delta \mathrm{V}_{\theta}^{2}}{\mathrm{U}^{2}}\right)
$$

The first term on the right-hand side of equation $6.1, \Delta \mathrm{r} / \mathrm{r}$, controls the overall magnitude of the perturbation centrifugal forces in the stage. This shows that if a stage has a low span-to-radius ratio, $\Delta \mathrm{r} / \mathrm{r} \rightarrow 0$, then the perturbation centrifugal forces in the boundary layer approach zero. This term can also be rewritten as the hub to tip ratio of the compressor. The second term on the right-hand side of equation $6.1, \Delta \mathrm{V}_{\theta}^{2} / \mathrm{U}^{2}$, varies across the blade surfaces and is a measure of the relative local magnitude of the perturbation centrifugal forces in the boundary layer.

For the rotor this second term can be written as:

$$
\frac{\Delta \mathrm{V}_{\theta}^{2}}{\mathrm{U}^{2}}=\frac{\mathrm{U}^{2}-\left(\mathrm{V}_{\theta}^{2}\right)_{\mathrm{fs}}}{\mathrm{U}^{2}}
$$

because the fluid on the rotor blade surface moves at the blade velocity. For the stator, it can be written as: 


$$
\frac{\Delta \mathrm{V}_{\theta}^{2}}{\mathrm{U}^{2}}=\frac{0-\left(\mathrm{V}_{\theta}^{2}\right)_{\mathrm{fs}}}{\mathrm{U}^{2}}
$$

because the fluid on the stator blade surface is stationary.

Each term in equations 6.2 and 6.3 is plotted in the upper half of Figure 6.8. The red dashed lines show the square of the blade speed of the rotor $\mathrm{U}^{2}$ and the stator, 0 . The lower half of Figure 6.8 shows the overall magnitude of the terms in equations 6.2 and 6.3. The lower half of Figure 6.8 shows that, as expected, the perturbation centrifugal forces in the rotor and stator have opposite signs. They are radially outward in the rotor and radially inward in the stator. However, the important point to note is that perturbation centrifugal forces in the rotor are between two and four times larger than in the stator. The cause of this difference is the difference in the tangential velocity squared, shown in equations 6.2 and 6.3. Finally, Figure 6.9 shows how raising reaction changes the magnitude of the differential boundary layer effects caused by rotation.

We can now understand the answer to the first question. Rotation extends the operating range of the stage through two mechanisms. First, the perturbation centrifugal forces, $F_{c}$, in the rotor, result in a radially outward pumping of low momentum fluid which delays the onset of separation in the rotor. Second, the perturbation centrifugal forces in the rotor reduce the deficit in dynamic pressure in the repeating stage endwall boundary layer into the stator hub, and this delays the onset of the hub corner separation in the stator. This is not caused by the perturbation centrifugal forces in the stator but instead by the forces in the rotor which pump low momentum fluid radially outwards. This explains why in Figure 6.7 rotation acts to reduce the size of the stator hub separation. 

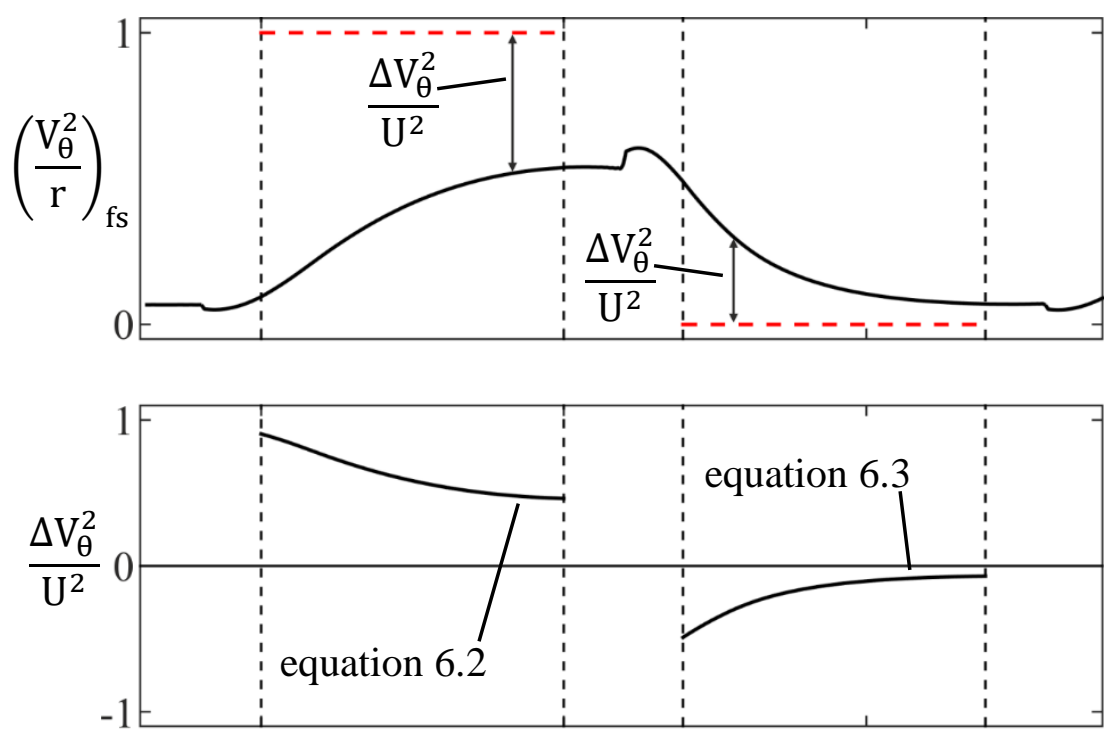

Surface Distance

Figure 6.8: Differential boundary layer effect of rotation for 50\% reaction (3-D CFD).

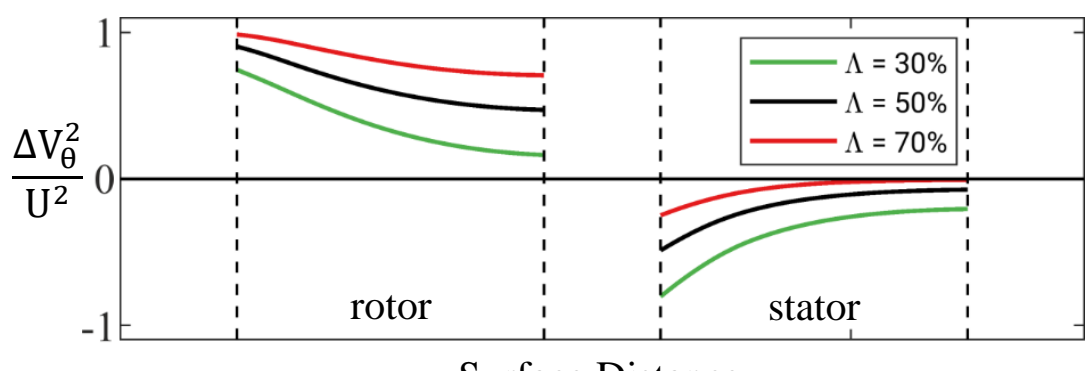

Surface Distance

Figure 6.9: Differential boundary layer effect of rotation (3-D CFD).

We can now return to the second question, why the extension in operating range is larger at high reaction. We will use the framework of the dimensionless perturbation centrifugal force $F_{c}$, outlined in this section, to answer this.

\subsection{Why does rotation extend operating range further at high reaction?}

We can now explain the cause of the large increase in maximum pressure rise coefficient at high reaction. Figure 6.10 shows the effect of rotation on the stator limiting streamlines. 
Rotational effects increase the maximum pressure rise by reducing the size of the stator separation.

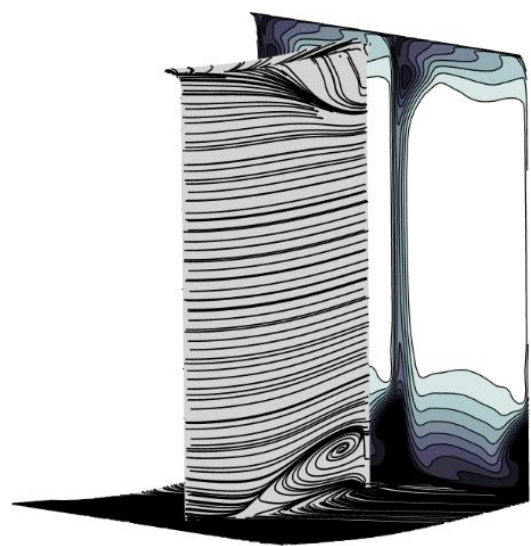

Rotation Off

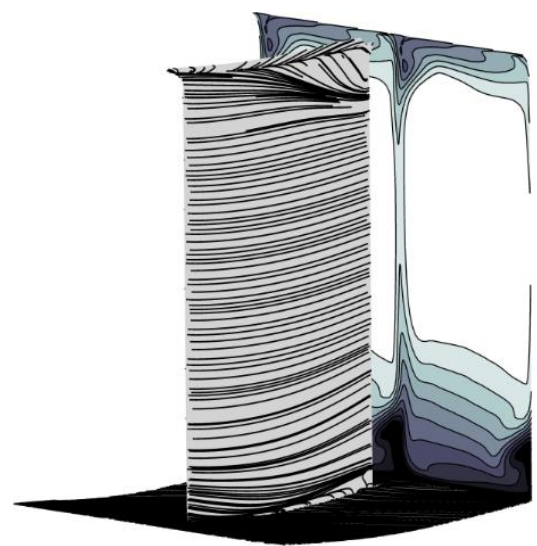

Rotation On

Figure 6.10: Stator suction-surface limiting streamlines for $70 \%$ reaction without clearances (3-D CFD).

The cause of this reduction can be seen in Figure 6.11. The rotational effects in the rotor causes a radial migration of high loss fluid in the hub towards the midspan. This in turn increases the endwall dynamic pressure entering the stator hub. This causes the local static-pressure rise coefficient in the stator hub, shown in Figure 6.11, to drop. It can be seen from Figure 6.9 that as reaction rises, the perturbation centrifugal force, $\mathrm{F}_{\mathrm{c}}$, in the rotor rises. This explains why this mechanism acts to increase the maximum pressure rise coefficient of the compressor at high reaction.

We have now understood the answer to the second question. A simple schematic is shown in Figure 6.12 which describes this mechanism. This behaviour is fundamental to all cantilever compressors and shows that as reaction is raised, there is a natural asymmetry to the way in which the perturbation centrifugal force extends the operating range of the compressor. 


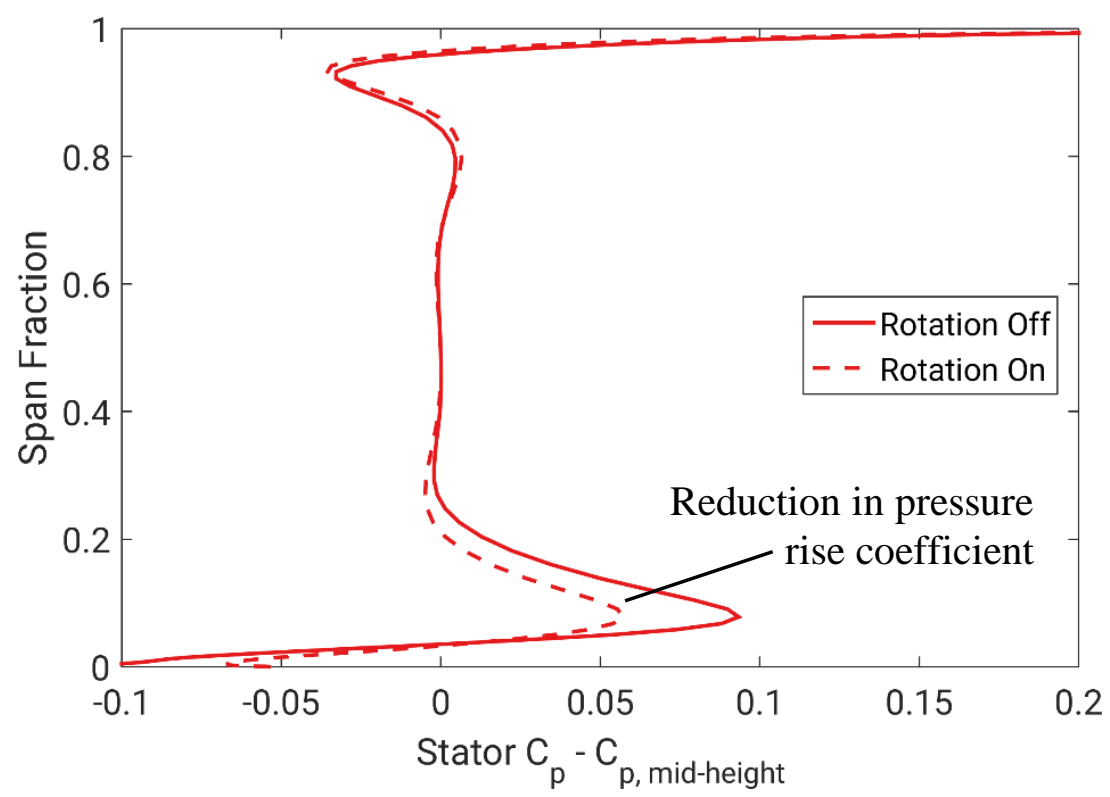

Figure 6.11: Spanwise variation of stator static-pressure rise for case of $70 \%$ reaction (3-D CFD).

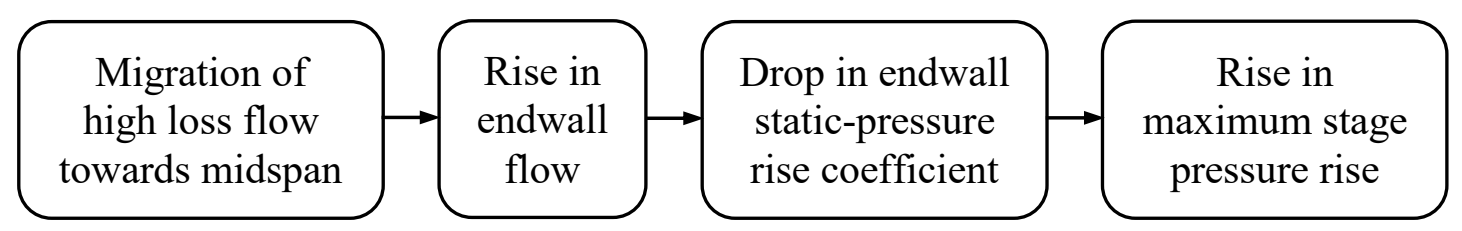

Figure 6.12: Mechanism rotational effects increase the maximum pressure rise of a stage.

\subsection{Effect of varying level of rotation}

The effect of varying the level of rotation on the design loss of the compressor is shown in Figure 6.13. The effective radius $r_{\text {eff }}$ in the rotation model has been varied between a relatively high value, corresponding to a low value on the $\mathrm{x}$-axis, where the blades act as if part of a rectilinear cascade, and a relatively low value, corresponding to a high value on the $\mathrm{x}$-axis. Where the effective radius is set equal to the radius of a real compressor $(100 \%)$, the model imposes the differential boundary layer effects of rotation that are expected in a real compressor. 
The figure shows the effect of changing the effective radius of the compressor from $0.03 \%$ of a real compressor (a rectilinear cascade) to $250 \%$ of a real compressor. As expected from the findings presented in Section 6.1, the figure shows that the effect of changing the effective radius of the compressor is small. As the effective radius changes between approximately $25 \%$ and $125 \%$ of a real compressor, the efficiency drops by just $0.05 \%$, for the case without clearances and $0.16 \%$ with clearances.

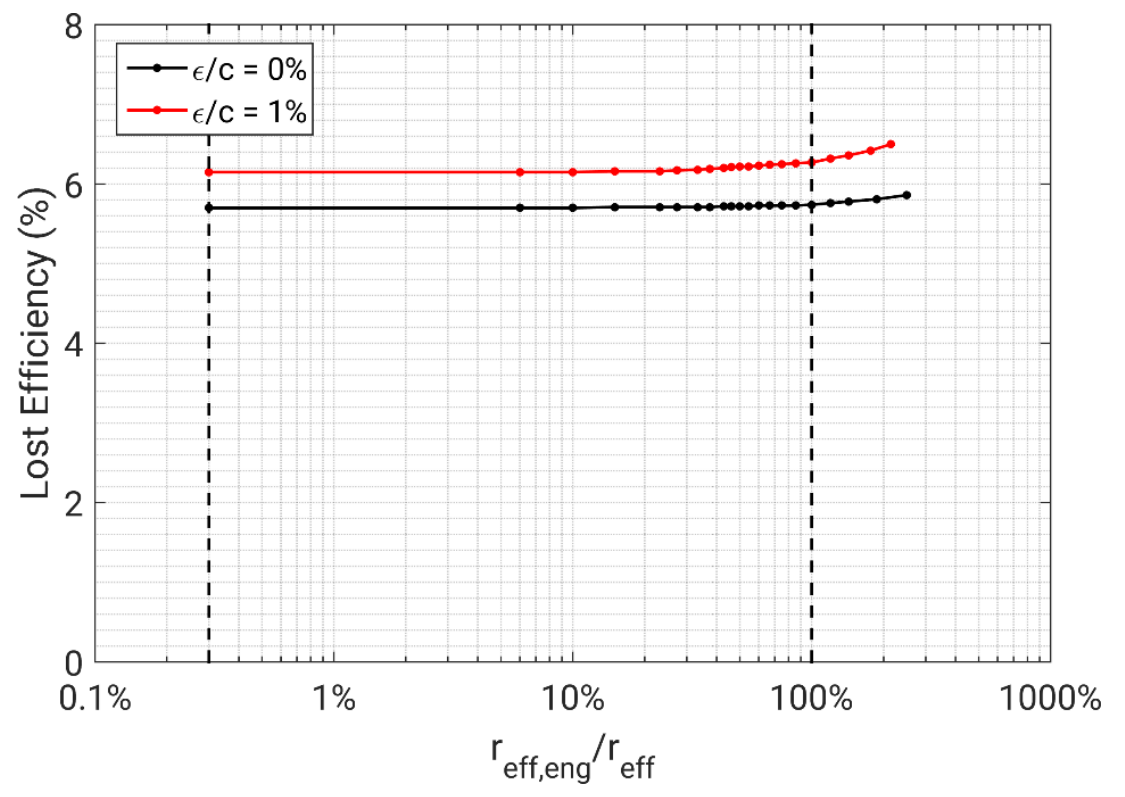

Figure 6.13: Lost efficiency for cases with varying level of rotation (3-D CFD).

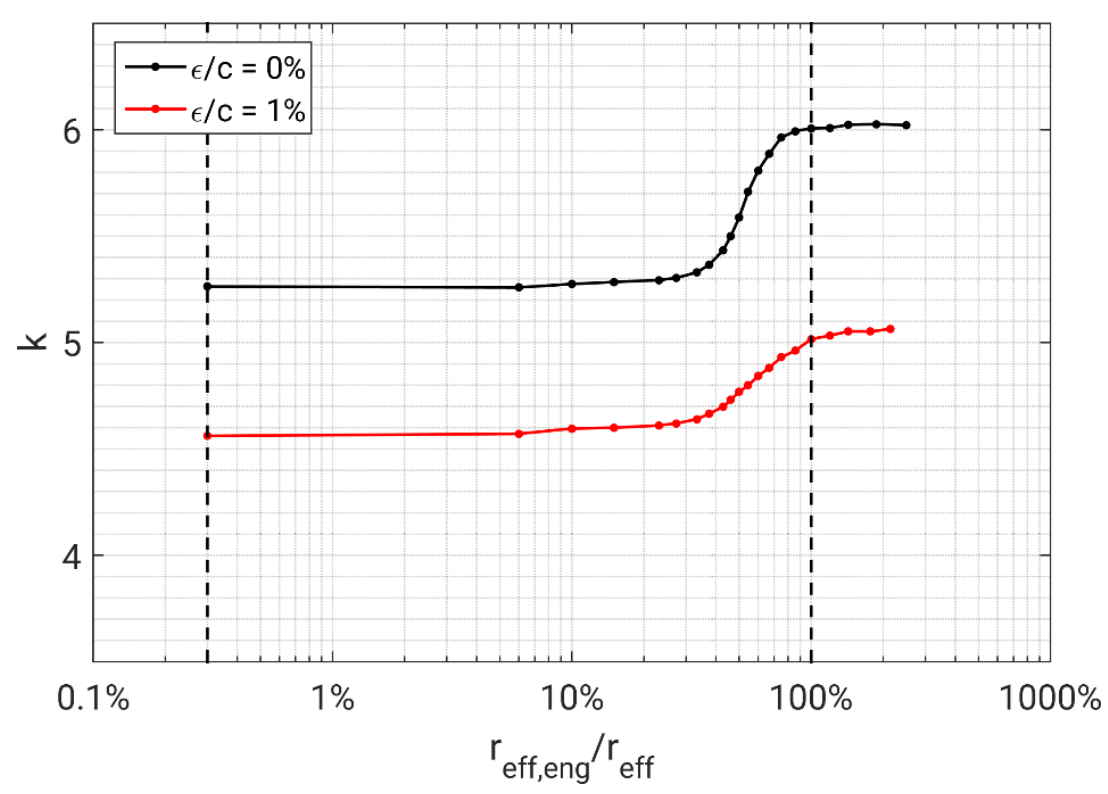

Figure 6.14: Throttle coefficients for cases with varying level of rotation (3-D CFD). 
The effect of varying the level of rotation on the maximum pressure rise of the compressor is shown in Figure 6.14. The figure shows the effect of changing the effective radius of the compressor from $0.03 \%$ of a real compressor (a rectilinear cascade) to $250 \%$ of a real compressor. The figure shows that the increase in the maximum pressure rise occurs when the effective radius changes between approximately $25 \%$ and $125 \%$ of a real compressor.

\subsection{Summary}

The effect of rotation on the design efficiency of the stage was found to be relatively small. However, the reaction which achieves the optimal design efficiency was found to increases by around 5\% reaction (from 50\% reaction to $55 \%$ reaction). The range of reactions over which the efficiency only varies by $0.01 \%$ was relatively wide, between $50 \%$ reaction and $60 \%$ reaction. Rotation was found to have a negligible effect on profile loss and was found to remain almost invariant with reaction.

The principal effect of rotation on the compressor is to improve its operating range. Furthermore, rotation was found to increase the reaction which achieves the maximum pressure rise, by around $15 \%$ (from $50 \%$ reaction to $65 \%$ reaction). This is brought about by the perturbation centrifugal forces in the rotor which introduce a natural asymmetry. It is critical to consider this effect in preliminary design. This effect is not captured in the correlations currently used in preliminary design. 


\section{Chapter 7}

\section{Application to multistage compressors}

In this chapter, the understanding developed in the previous three chapters is applied to show how the choice of reaction affects the overall performance of a multistage compressor.

The inlet swirl to a compressor stage and its reaction are coupled. If the flow into a stage is axial, then the stage reaction is automatically high. This chapter investigates different approaches which the designer can take, to deal with this high reaction. The chapter is divided into four parts. First, the stagewise and spanwise distributions of reaction in the compressor are discussed, in turn. Low reaction compressor are investigated in the third part and at the end of the chapter, there is a summary.

\subsection{Stagewise distribution of reaction}

We are now in a position to understand how the choice of reaction affects the overall lost efficiency of a multistage core compressor. This is an important industry question because the requirement for axial flow at the inlet and exit of a multistage compressor naturally results in high reaction. The designer therefore must decide whether to tolerate this high reaction through the compressor or to aim for a more optimal reaction in the central stages of the compressor.

To answer this question the lost efficiency of a multistage machine can be written as: 


$$
\left(\frac{\mathrm{T} \Delta \mathrm{s}}{\Delta \mathrm{h}_{0}}\right)_{\text {compressor }}=\frac{\sum_{1}^{\mathrm{n}}(\mathrm{T} \Delta \mathrm{s})_{\text {stage }}}{\Delta \mathrm{h}_{0}}
$$

where the summation of loss is across all $\mathrm{n}$ stages and $\Delta \mathrm{h}_{0}$ is the isentropic work input to the machine. We will consider a hypothetical $n=10$ stage compressor, with axial flow at the inlet and exit. For conventional levels of work $\left(\Psi_{d}=0.436\right)$ and flow coefficient $\left(\Phi_{\mathrm{d}}=0.597\right)$, axial flow at the inlet and exit of the compressor corresponds to a reaction of approximately $75 \%$. The lost efficiencies found in the Chapter 6 can be used in equation 7.1 to estimate the overall lost efficiency of a multistage compressor.

To understand how the choice of reaction affects the overall lost efficiency we will consider three cases. The stagewise distribution of reaction in these three cases is shown in Figure 7.1, for the 10 stage compressor.

\subsubsection{0\% reaction compressor}

Case A represents a historic design philosophy of having 50\% reaction in all the stages. This design choice was adopted widely in the past. It is advocated in many turbomachinery design textbooks. To achieve 50\% reaction in all the stages, an Inlet Guide Vane (IGV) and Outlet Guide Vane (OGV) is required. This adds additional loss. We will consider the loss coefficient of the IGV and OGV to be 0.04 , based on the correlations of Lieblein (1959).

\subsubsection{High reaction compressor}

Case B represents a second historic design philosophy where the reaction is maintained at $75 \%$ through all stages. This benefits from having no IGV or OGV, however, it suffers from having a reaction which has a higher design loss. This was an appealing alternative to Case $\mathrm{A}$ in the past, in particular, in low bypass compressors. This design also benefits from having similar stages, which greatly simplifies the stage design and manufacture.

This case was found to have a design efficiency which is $0.07 \%$ higher than case A. This is because the efficiency gain caused by removing the IGV and OGV is approximately offset by the rise in reaction. It should be noted that this design philosophy 
would have a better operability than Case A, due to the increased maximum pressure rise of its stages. This could explain why many historic compressors, with high reactions, had a relatively good design efficiency and operating range.
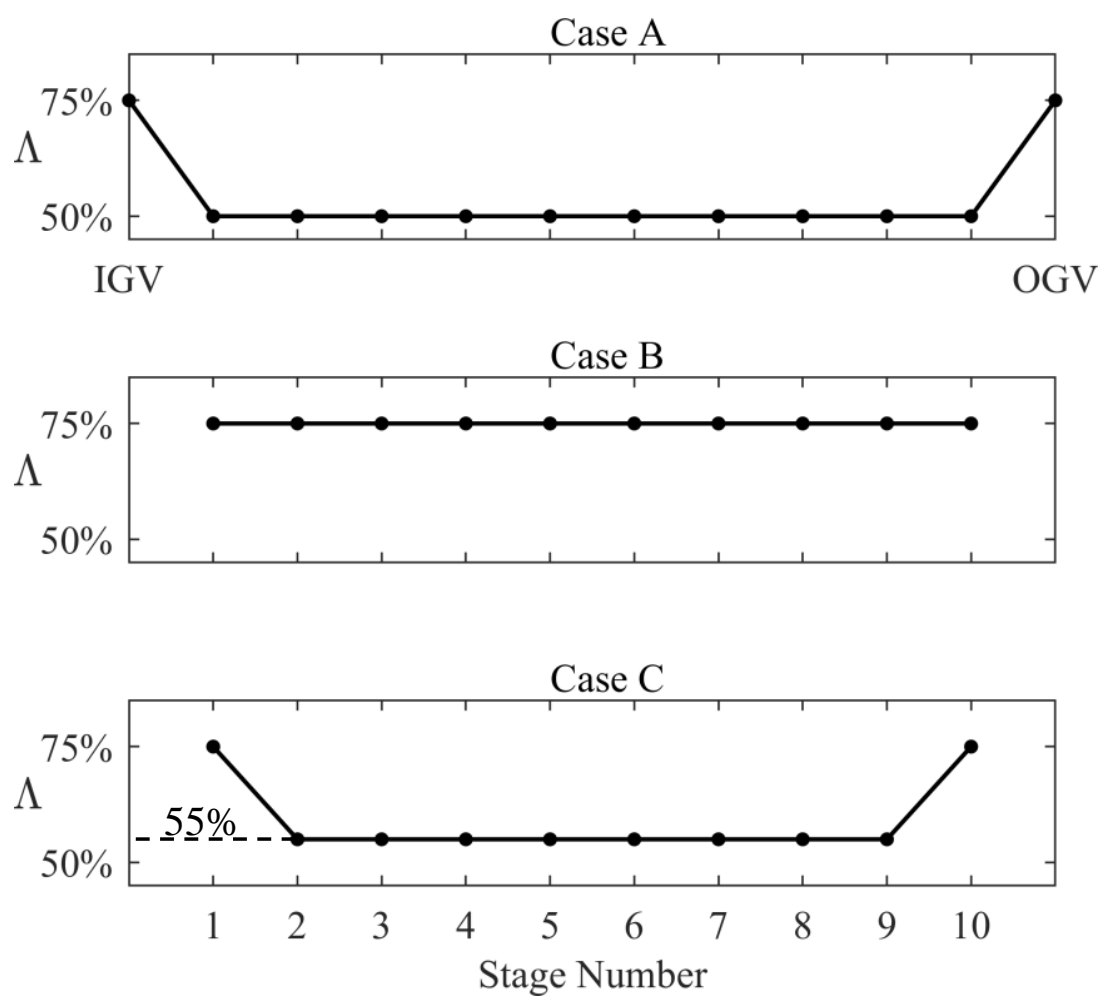

Figure 7.1: Comparison of three stagewise distributions of reaction for a 10 stage compressor.

\subsubsection{Maximum design efficiency compressor}

Case $\mathrm{C}$ represents the compressor with the maximum design efficiency. This compressor has central stages which have a reaction of $55 \%$, as shown in section 6.1 . This was found to result in a design efficiency which was $0.65 \%$ higher than case A.

It is therefore clear that, if maximising efficiency is the aim of the designer, then the reaction of the central stages should be $55 \%$. However, it is important to note that if the central stages have an efficiency between $50 \%$ and $60 \%$ the change in efficiency is relatively small. This provides the designer with a useful degree of freedom. 


\subsection{Spanwise distribution of reaction}

This section looks at how the variation in reaction up the span of the blade affects performance. At low hub-to-tip ratios, there is a significant difference between the rotor blade speed at the hub and at the tip. This results in a changing velocity triangle design, up the span of the compressor. This in turn changes the variation in work coefficient, flow coefficient and reaction up the blade span, and therefore the performance of the compressor. It is therefore important for a designer to be able to have a low order understanding of how the spanwise variation of reaction effects performance.

Three different ways of fixing the spanwise variation of reaction up the blade span are discussed in this section, in turn. These represent the case of constant work and flow, constant reaction, and a tailored reaction profile.

To understand how the spanwise choice of reaction affects the performance of the compressor, it is first necessary to consider the radial equilibrium of the flow. In this section the compressor stages have a plane annulus and the blades operate at near the design point. A radial equilibrium equation for incompressible flow can therefore be written as:

$$
\frac{1}{\rho} \frac{\mathrm{dp}_{0}}{\mathrm{dr}}=\mathrm{V}_{\mathrm{x}} \frac{\mathrm{dV} \mathrm{V}_{\mathrm{x}}}{\mathrm{dr}}+\frac{\mathrm{V}_{\theta}}{\mathrm{r}} \frac{\mathrm{d}\left(\mathrm{rV}_{\theta}\right)}{\mathrm{dr}}
$$

where $\mathrm{p}_{0}$ is the stagnation-pressure at each location up the span. The full derivation of equation 7.2 is given in Appendix B. The first term in equation 7.2 includes the radial distribution of work, $\mathrm{dh}_{0} / \mathrm{dr}$, and losses, $\mathrm{ds} / \mathrm{dr}$. The third term changes, depending on the chosen vortex flow, as it affects the way in which the radial momentum of the flow, $\mathrm{rV}_{\theta}$, changes with radius. It is clear from equation 7.2 therefore that the second term, the axial velocity profile, is set by the first and third terms.

In a repeating stage, the reaction at each radius can be written as:

$$
1-\Lambda=\frac{\mathrm{V}_{\theta 1}+\mathrm{V}_{\theta 2}}{2 \Omega \mathrm{r}}
$$


where $V_{\theta 1}$ and $V_{\theta 2}$ are the absolute tangential velocity into the rotor and the stator. It is clear from equation 7.3 that the reaction sets the level of interstage swirl of the stage. Equation 7.3 is easily derived from the common repeating stage velocity triangle relationships, derived in most turbomachinery design textbooks, for example Dixon \& Hall (2014).

It is clear from equation 7.3 that for a chosen type of vortex flow, which represents a rule for setting $V_{\theta}$, the shape of the radial distribution of reaction is fixed. This choice of vortex flow also fixes the third term in equation 7.2. Therefore if the work input is known in equation 7.2, the axial velocity profile can be calculated. Although it is not strictly necessary to design the stage to satisfy radial equilibrium in this way, it is practical to do so. The observations of Smith (1969), described in Section 3.4, show that after the first few stages in a multistage compressor the axial velocity profile reaches a steady profile and uniform work is done at up the span. It is therefore practical to design a stage by following equation 7.2.

We can now look at the three different ways of fixing the spanwise variation of reaction up the blade span. In each case we will consider the impact on the design loss and operating range of the compressor.

\subsubsection{Constant work and flow}

If the work input $\Delta \mathrm{h}_{0}$ is to be uniform radially, as the blade speed is proportional to the radius, the change in absolute tangential velocity $\Delta V_{\theta}$ across the rotor must be inversely proportional to the radius. This is the definition of a free vortex design. This design choice was advocated widely in the past, Cumpsty (1989). It is defined by:

$$
\begin{aligned}
& V_{\theta 1}=\frac{k_{1}}{r} \\
& V_{\theta 2}=\frac{k_{2}}{r}
\end{aligned}
$$


where $\mathrm{k}_{1}$ and $\mathrm{k}_{2}$ in equations 7.4 and 7.5 are constants and $\mathrm{r}$ is the radius. Combining equations 7.4 and 7.5 with equation 7.3 gives:

$$
1-\Lambda=\left(\frac{\mathrm{k}_{1}+\mathrm{k}_{2}}{\Omega}\right) \frac{1}{\mathrm{r}^{2}}
$$

which shows that the reaction is inversely proportional to the square of the radius. The level of variation of reaction up the span is controlled by the hub-to-tip ratio, $r_{h} / r_{t}$.

For conventional levels of work $\left(\Psi_{\mathrm{d}}=0.436\right)$ and flow coefficient $\left(\Phi_{\mathrm{d}}=0.597\right)$, the findings of Chapter 6 concluded that the compressor which achieves the maximum design efficiency, has a reaction of 55\%. The designer may therefore choose a reaction of $55 \%$, along the meanline of the compressor. The spanwise variation in reaction, for the free vortex design described by equation 7.6, is shown in Figure 7.2, for this case. The black line shows the case of $r_{h} / r_{t}$ equal to 0.8 . It can be seen that low reaction results at the hub and high reaction at the casing. The findings of Chapter 6 imply that to a first order, for the case without clearances, this drops the total endwall lost efficiency by $0.1 \%$, compared to the case of a constant reaction of 55\% up the span. The hub reaction drops even further as the $r_{h} / r_{t}$ drops to 0.6, shown in red in Figure 7.2. The findings of Chapter 6 indicate that this low reaction at the hub is undesirable for operating range.

If the designer wishes to design a stage with a good operating range then Chapter 6 shows that a high reaction design in the hub region is required. The findings of Chapter 6 concluded that the compressor which achieves the maximum operating range, has a reaction of approximately $65 \%$. The designer may therefore choose a reaction of $65 \%$, along the hub of the compressor. The spanwise variation in reaction, for the case of $65 \%$ hub reaction is shown in Figure 7.3. The reaction does not drop below 65\%. This increases the total endwall lost efficiency by $0.3 \%$, compared to the case of a constant reaction of $55 \%$, but the stage benefits from a good operating range. 


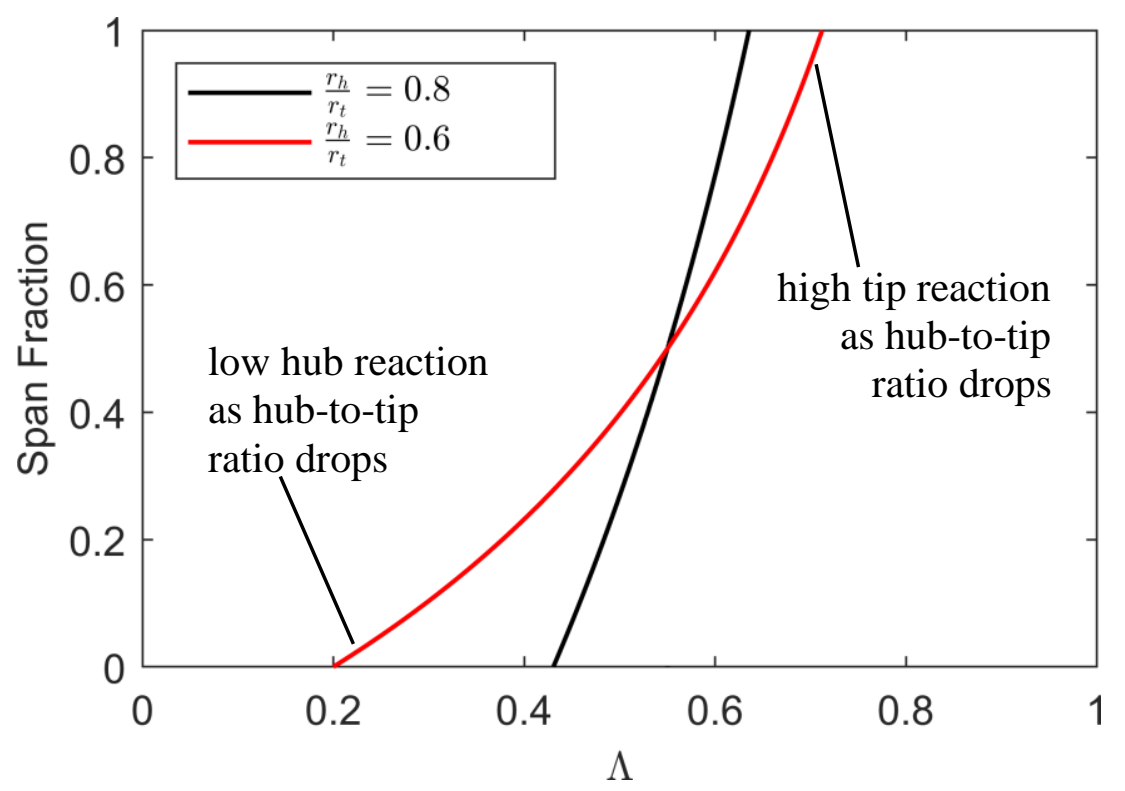

Figure 7.2: Spanwise variation in reaction for a free vortex design (equation 7.6), where meanline reaction is set to $55 \%$.

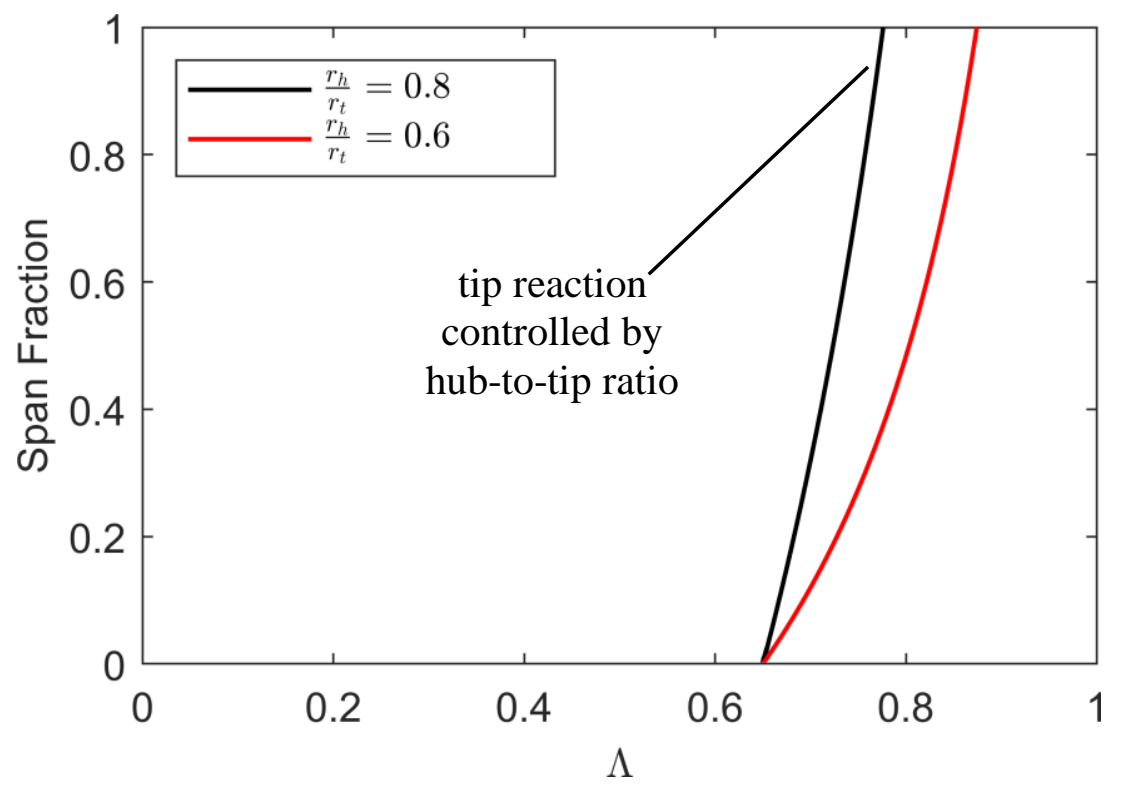

Figure 7.3: Spanwise variation in reaction for a free vortex design (equation 7.6), where hub reaction is set to $65 \%$.

If the designer therefore wishes to hold the work and flow constant up the blade span, allowing the reaction to vary, the compressor with the best design efficiency has a high reaction along the casing, and low reaction along the hub. However this compressor has 
a poor operating range. To overcome this problem the designer should aim for a higher reaction along the hub of the compressor.

\subsubsection{Constant reaction}

If instead the absolute tangential velocities vary proportionally with the radius, a stage of approximately constant spanwise reaction can be designed. In the case of a forced vortex, the absolute tangential velocities into the rotor and stator, $V_{\theta 1}$ and $V_{\theta 2}$, are given by:

$$
\begin{aligned}
\mathrm{V}_{\theta 1} & =\mathrm{c}_{1} \mathrm{r} \\
\mathrm{V}_{\theta 2} & =\mathrm{c}_{2} \mathrm{r}
\end{aligned}
$$

where $c_{1}$ and $c_{2}$ in equations 7.7 and 7.8 are constants. Combining equations 7.7 and 7.8 with equation 7.3 gives:

$$
1-\Lambda=\left(\frac{\mathrm{c}_{1}+\mathrm{c}_{2}}{\Omega}\right)
$$

which shows that in the case of a forced vortex design, the reaction is constant with radius.

This stage benefits from the fact that work coefficient $\Psi$ can be held constant up the span. It is also possible for the axial velocity $V_{x}$ to be held approximately constant in equation 7.2 for this case. This is because in the forced vortex design, the first term and the third term in equation 7.2 both vary with $r^{2}$.

If the designer therefore aims to maximise the design point efficiency of the compressor, a reaction of $55 \%$ should be chosen, based on the findings in Chapter 6 . If instead the designer wishes to maximise the operating range of the compressor, a reaction of $65 \%$ should be chosen. 


\subsubsection{Tailored reaction profile}

The design efficiency of the compressor was shown to rise by choosing a high reaction along the casing, and low reaction along the hub. However the low reaction along the hub gives this compressor a poor operating range. To overcome this, what is required is a way to tailor the reaction up the span, so that the designer has more control.

To produce the radially constant work input $\Delta \mathrm{h}_{0}$, as in Section 7.2.1, it is only necessary that the difference in absolute tangential velocity across the rotor, $\Delta V_{\theta}=V_{\theta 2}-$ $\mathrm{V}_{\theta 1}$, is proportional to the radius. One historic family of designs which achieves this is to choose:

$$
\begin{aligned}
& \mathrm{V}_{\theta 1}=a r^{\mathrm{n}}-\frac{\mathrm{b}}{\mathrm{r}} \\
& \mathrm{V}_{\theta 2}=a r^{\mathrm{n}}+\frac{\mathrm{b}}{\mathrm{r}}
\end{aligned}
$$

where $\mathrm{a}$ and $\mathrm{b}$ in equations 7.10 and 7.11 are constants and $\mathrm{n}$ is an index which can be either 0 or 1 . Combining equations 7.10 and 7.11 with equation 7.3 gives:

$$
1-\Lambda=\left(\frac{\mathrm{a}}{\Omega}\right) \mathrm{r}^{\mathrm{n}-1}
$$

which shows that the variation in reaction is proportional to the term $\mathrm{r}^{\mathrm{n}-1}$.

For the case of $n=0$, equation 7.12 shows that the variation in reaction is inversely proportional to the radius. As in section 7.2.1, the designer may choose a reaction of $55 \%$, along the meanline of the compressor. The spanwise variation in reaction, for the vortex design described by equation 7.12, is shown in Figure 7.4, for this case. In this design, the reaction varies a little with radius but change in reaction at the hub and at the casing is not excessive. For the case of $r_{h} / r_{t}$ equal to 0.8 and 0.6 , this drops the total endwall lost efficiency by $0.05 \%$ and $0.2 \%$, with little effect on the operating range of the compressor, compared to the case of a constant reaction of $55 \%$. 
In addition, it is straightforward to show that the vortex designs described by equation 7.10 and 7.11 satisfy equation 7.2 and therefore it is possible that the axial velocity $\mathrm{V}_{\mathrm{x}}$ be held approximately constant with radius.

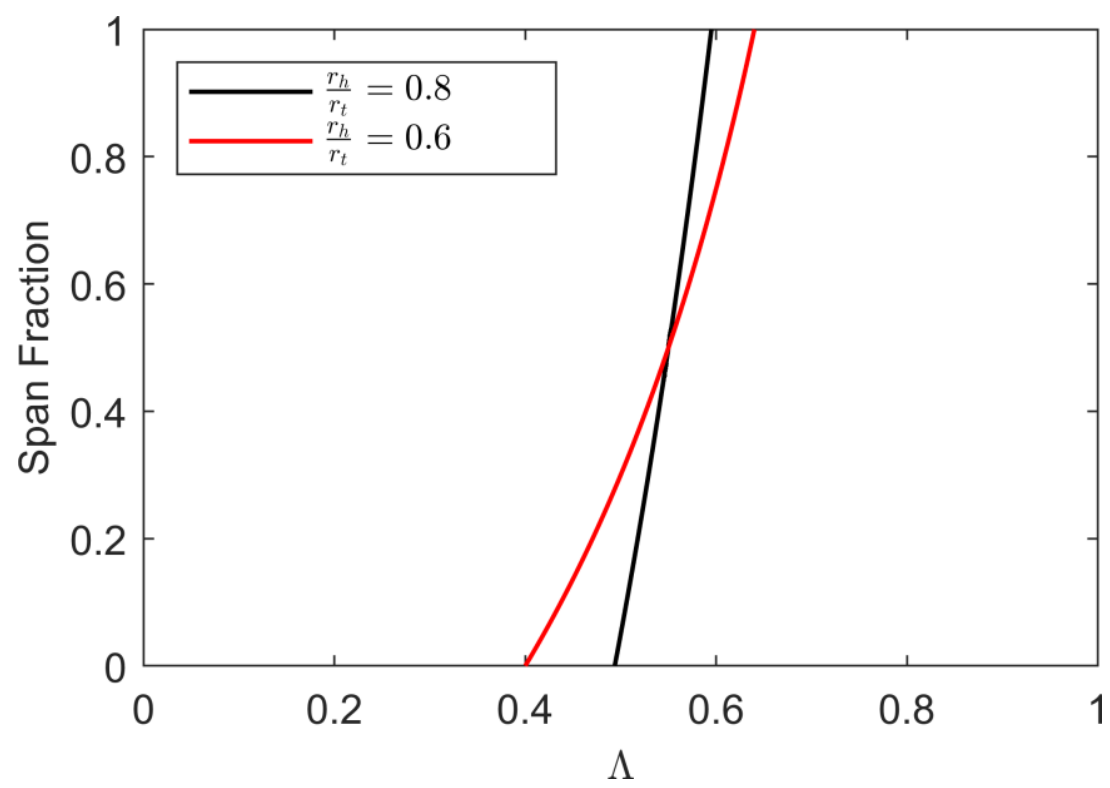

Figure 7.4: Spanwise variation in reaction for $n=0$ design (equation 7.12), where meanline reaction is set to $55 \%$.

For the case of $n=1$, equation 7.12 reduces to the forced vortex design, described in section 7.2.2, with $\mathrm{a}=\mathrm{c}_{1}+\mathrm{c}_{2}$.

The choice of a tailored reaction profile therefore allows the designer improve the design efficiency of the compressor, without decreasing its operating range.

A compressor designed with high reaction at the casing and low reaction at the hub has the best design efficiency. However, choosing a low reaction at the hub, gives this compressor a poor operating range. A designer should therefore aim for a relatively higher reaction at the hub, and control the variation of reaction up the span using a 'tailored reaction profile'. It is important to note that the maximum efficiency is a weak function of reaction between reactions of $50 \%$ and $60 \%$, which provides a useful degree of freedom to the designer, when tailoring the reaction profile. 


\subsection{Low reaction compressors}

Compressors of reaction less than $50 \%$ are rarely designed. One reason why this may be the case is that low reaction compressors must sustain a large radial pressure gradient. The reason for this can be understood by considering the radial equilibrium of the flow.

The lower the reaction, the greater the absolute tangential velocity $V_{\theta}$, relative to the rotor blade speed U. This can be understood from Figure 7.5. The figure shows a schematic of the time averaged mid-height streamline in the absolute frame for $30 \%$ and $70 \%$ reaction stages.

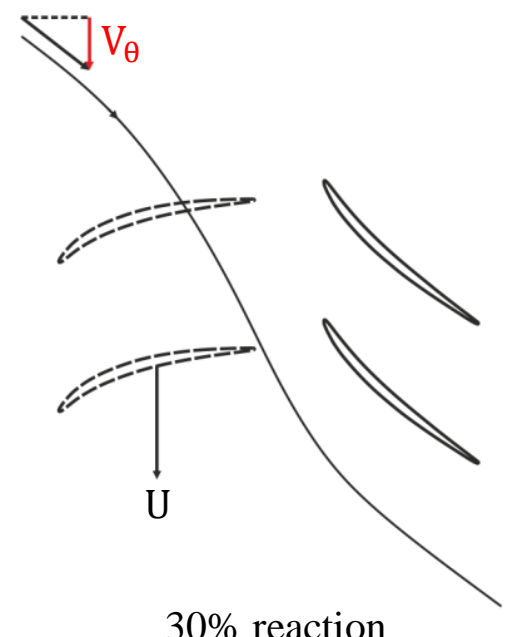

$30 \%$ reaction

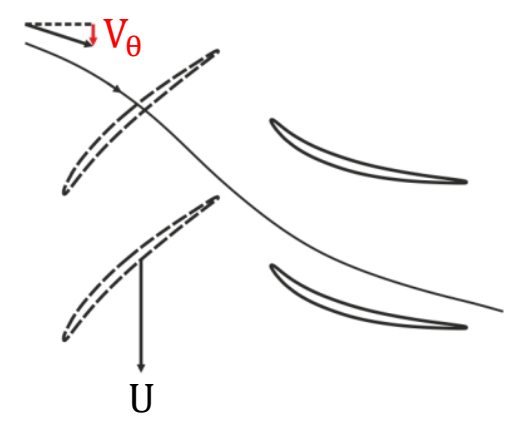

$70 \%$ reaction

Figure 7.5: Mid-span streamline in absolute frame of reference for $30 \%$ reaction (left) and $70 \%$ reaction (right) stages.

Dropping the reaction can be seen to increase $V_{\theta}$, for a constant $U$, through the stage. To satisfy radial equilibrium, the radial component of the static-pressure gradient must therefore rise, as the reaction drops. This can be understood by looking at equation B2. This large radial pressure gradient can lead to excessive spatial gradients in the spanwise flow conditions. 
Another reason that compressors of reaction less than 50\% are rarely designed is that they require high turning Inlet Guide Vanes (IGVs) and Outlet Guide Vanes (OGVs). In a low reaction stage, the absolute swirl at the inlet and exit of the compressor is high. This means that IGVs and OGVs are required to swirl and deswirl the flow, from and to the axial direction. The additional losses due to theses high turning IGVs and OGVs are clearly diminish the efficiency of the compressor.

Compressors designed with a reaction of less than $50 \%$ have large radial pressure gradients and require high turning IGVs and OGVs, which add loss. They are therefore seldom designed.

\subsection{Summary}

In a multistage compressor the requirement for axial flow at the inlet and exit of the multistage compressor results in high reaction. The designer can choose to tolerate this high reaction through the compressor or aim for a more optimal reaction in the central stages. The lost efficiencies found in the Chapter 7 were used to estimate the overall efficiency of a hypothetical $n=10$ stage compressor. It was shown that if the compressor with a constant stage reaction of $75 \%$, was rebladed so that the central 8 stages had $55 \%$ reaction, then the overall design point efficiency would rise by $0.58 \%$.

The compressor with the highest design efficiency should have high reaction along the casing, and low reaction along the hub. However, at low hub-to-tip ratios, the reaction at the hub can be extremely low, which is gives the compressor a poor operating range. To overcome this problem the designer should choose an optimal reaction of $55 \%$ for design loss, along the meanline of the compressor, and aim for a higher reaction at the hub. For a hub-to-tip ratio of 0.6, the lost efficiencies found in the Chapter 6 were used to show that for a 'tailored reaction profile', this drops the total endwall lost efficiency by $0.2 \%$, with little effect to operating range, compared to the case of a constant reaction of 55\%. If instead the designer wishes to maximise the operating range of the compressor, a constant reaction of $65 \%$ should be chosen. It is important to note that between reactions of $50 \%$ and $60 \%$, the efficiency of the compressor is a weak function of reaction. 


\section{Chapter 8}

\section{Conclusions}

This thesis examined the effect of reaction on the efficiency and operating range of the compressor. The major findings from this work are summarised, and the consequential recommendations for future work are discussed in turn, in this chapter.

\subsection{Major findings}

There is considerable debate over the effect of reaction on compressor design efficiency and operating range. This study shows that the confusion is due in part to the inability to decouple the effects of the centrifugal force and the effects of changing the velocity triangle, in a controllable way.

A unique approach has been taken in which the centrifugal forces have been removed by using McKenzie's concept of a 'linear repeating stage', McKenzie (1997). The perturbation centrifugal force has then been reintroduced using a body force. This has allowed the two asymmetries, centrifugal force and velocity triangle, to be decoupled and has allowed their effect on compressor performance to be studied independently for the first time. The ability to accurately decouple these two asymmetries has led to a number of major findings. 


\subsubsection{Optimal reaction}

The effect of reaction on profile loss has been shown to be highly dependent on the methodology by which the solidity is set. When the solidity is set by the shape factor of the suction-surface boundary layer at the blade trailing edge, and conventional levels of work and flow coefficient are used $\left(\Psi_{\mathrm{d}}=0.44\right.$ and $\left.\Phi_{\mathrm{d}}=0.60\right)$, the profile loss has been shown to be relatively independent of reaction.

Reaction is shown to have a major effect on endwall loss. This is because it controls the freestream velocity of the flow relative to the endwall, at the edge of the endwall boundary layer. When the centrifugal effects are removed. this results in 50\% reaction compressors having the lowest endwall loss and thus the highest design efficiency.

A surprising conclusion of the study is that the maximum pressure rise capability of high reaction compressors is limited not by the rotor, but by the stator. This is counterintuitive because at high reaction the pressure rise in the rotor is greater than in the stator. The cause of this is due to the way reaction changes endwall loss, and the way reaction changes the re-energising effect provided by the change in reference frame, described by Koch (1981) and Auchoybur and Miller (2017).

When the centrifugal forces are reintroduced, the compressor with the maximum design efficiency is found to rise in reaction by $5 \%$ (from $50 \%$ reaction to $55 \%$ reaction) and the compressor with the maximum operating range is found to rise in reaction by $15 \%$ (from $50 \%$ reaction to $65 \%$ reaction). If a designer aims to maximise the design efficiency of a compressor, the reaction of its central stages should therefore be $55 \%$. However, it is important to note that the maximum efficiency is a weak function of reaction between reactions of $50 \%$ and $60 \%$.

\subsubsection{Impact on compressor design}

It is worth considering the impact of the study on design. Currently many preliminary design systems do not differentiate between rotors and stators in terms of loss and operating range. This means they are unlikely to correctly predict the true optimal reaction. Second the asymmetry between centrifugal forces in the rotor and stator 
boundary layers implies that three-dimensional blade design philosophies of rotating and stationary blades should differ.

The study shows that Roy Smith's statement that reactions higher than 50\% were optimal was correct. However, it has been shown that his reasoning was incorrect and that the primary cause is the asymmetry in the magnitude of the perturbation centrifugal forces in the rotor and stator boundary layers.

It is interesting to note that historically high reaction compressors operated with relatively high design efficiency and operating range. The study explains why this is the case. It is due to the higher centrifugal forces in the rotor acting as a form of boundary layer control.

It is also interesting to note that high reaction stages have lower optimal blade solidities. This significantly reduces the number of blades in the compressor. This implies that in the future, high reaction compressors could be optimal in applications where cost and weight are the primary drivers.

\subsection{Recommendations for future work}

The results of this study point to three potentially important areas of future work:

1. The trade between design loss and operating range.

2. The effect of Mach number, as the reaction is changed.

3. Exploiting the effects of rotation in 3-D rotor design.

\subsubsection{Improved design efficiency}

In this study, the effects of rotation have been shown to have a small effect on design loss, as the reaction is varied. However, the effects of rotation on the operating range of the compressor, are much larger. If instead of increased operating range, a designer intended to preserve operating range in exchange for improved design efficiency, then this could be achieved by removing blades. This trade should be studied in more detail. 


\subsubsection{Effect of Mach number}

The focus of this thesis has been to decouple the effects of reaction and Mach number. This was achieved by undertaking the study at low a Mach number. Now that the effect of reaction has been understood, it would be worth examining the effect of Mach number.

\subsubsection{Exploiting rotation effects in 3-D rotor design}

The asymmetry between the effects of rotation in the rotor and stator boundary layers implies that very different three-dimensional blade design philosophies should be undertaken in rotating and stationary blade rows. This should be studied in further detail. 


\section{References}

Auchoybur, K. (2017). Design of compressor endwall velocity triangles. $\mathrm{PhD}$ thesis: University of Cambridge, U.K.

Auchoybur, K., \& Miller, R. J. (2017). Design of compressor endwall velocity triangles. Journal of Turbomachinery, 139(6): 061005.

Banks, W. H., \& Gadd, G. E. (1963). Delaying effect of rotation on laminar separation. American Institute of Aeronautics and Astronautics Journal, 1(4): 941-942.

Baum, O., Koschichow, D., \& Fröhlich, J. (2016). Influence of the Coriolis force on the flow in a low pressure turbine cascade T106. ASME Turbo Expo Conference Proceedings. Seoul, South Korea: ASME paper GT2016-57399.

Casey, M. V. (1987). A mean line prediction method for estimating the performance characteristic of an axial compressor stage. International Conference on Turbomachinery: Efficiency Prediction and Improvement. Cambridge, U.K: IMechE Paper C264/87.

Cumpsty, N. A. (1989). Compressor aerodynamics. London: Longman Scientific \& Technical.

Denton, J. D. (1993). Loss mechanisms in turbomachines. Journal of Turbomachinery, 115(4), 621-656.

Denton, J. D. (2015). TBlock description and user manual version 15.1.

Dickens, T., \& Day, I. (2011). The design of highly loaded compressors. Journal of Turbomachinery, 133(3), 133(3): 031007.

Dixon, S. L., \& Hall, C. A. (2014). Fluid mechanics and Thermodynamics of Turbomachinery. Oxford: Butterworth-Heinemann. 
Drela, M., \& Youngren, H. (2008). A user's guide to MISES version 2.6.

Ekman, V. W. (1905). On the influence of the earth's rotation on ocean-currents. Arkiv för matematik, astronomi och fysik, 2(11): 1-52.

Farmakalides, C. D., McKenzie, A. B., \& Elder, R. L. (1994). The effect of reaction on axial flow compressor performance. ASME Turbo Expo Conference Proceedings. The Hague, Netherlands: ASME paper 94-GT-456.

Fogarty, L. E. (1951). The laminar boundary layer on a rotating blade. Journal of Aeronautical Sciences, 18(4), 18(4): 247-252.

Garzon, V. E., \& Darmofal, D. L. (2003). Impact of geometric variability on axial compressor performance . Journal of Turbomachinery, 125(4): 692-703.

Gbadebo, S. A., Cumpsty, N. A., \& Hynes, T. P. (2005). Three-dimensional separations in axial compressors. Journal of Turbomachinery, 127(2): 331-339.

Goodhand, M. N., \& Miller, R. J. (2011). Compressor leading edge spikes: A new performance criterion. Journal of Turbomachinery, 133(2): 021006.

Horlock, J. H. (1958). Axial flow compressors. London: Butterworths \& Co.

Horlock, J. H., \& Wordsworth, J. (1965). The three-dimensional laminar boundary layer on a rotating helical blade. Journal of Fluid Mechanics, 23(2): 305-314.

Hughes, D. W., \& Horlock, J. H. (1971). Effect of rotation on the development of the turbulent boundary layer. Proceedings of Salford Symposium on Internal Flows. London, U.K: IMechE paper 18.

Karimipanah, M. T., \& Olsson, E. K. (1992). Calculation of three-dimensional boundary layers on rotor blades using integral methods. Journal of Turbomachinery, 115(2): 342-353.

Klass, R., \& Kuhn, B. (1992). Fillet and surface intersections defined by rolling balls. Computer Aided Geometric Design Periodical, 9(3): 185-193.

Koch, C. C. (1981). Stalling pressure rise capability of axial flow compressor stages. Journal of Engineering for Gas Turbines and Power, 103(4): 645-656. 
Koch, C. C., \& Smith, L. H. (1976). Loss sources and magnitudes in axial-flow compressors. Journal of Engineering for Gas Turbines and Power, 98(3): 411424.

Kulfan, B. M. (2008). Universal parametric geometry representation method. Journal of Aircraft, 45(1): 142-158.

Lakshminarayana, B., \& Govindan, T. R. (1981). Analysis of turbulent boundary layer on cascade and rotor blades of turbomachinery. American Institute of Aeronautics and Astronautics Journal, 19(10): 1333-1341.

Lakshminarayana, B., Davino, R., \& Pouagare, M. (1982). Three-dimensional flow field in the tip region of a compressor rotor passage - Part I: Mean velocity profiles and annulus wall boundary layer. Journal of Engineering for Gas Turbines and Power, 104(4): 760-771.

Lakshminarayana, B., Jabbari, A., \& Yamaoka, H. (1972). Turbulent boundary layer on a rotating helical blade. Journal of Fluid Mechanics, 51(3): 545-569.

Lei, V. -M., Spakovszky, Z. S., \& Greitzer, E. M. (2008). A criterion for axial compressor hub-corner stall. Journal of Turbomachinery, 130(3): 031006.

Lieblein, S. (1953). Diffusion factor for estimating losses and limiting blade loadings in axial-flow-compressor blade elements. Cleveland, Ohio: NACA research memorandum.

Lieblein, S. (1959). Loss and stall analysis of compressor cascades. Journal of Fluids Engineering, 81(3): 387-397.

Mager, A., \& Hansen, H. (1952). Laminar boundary layer over flat plate. Cleveland, Ohio: NACA research memorandum.

McKenzie, A. B. (1997). Axial flow fans and compressors. London: Ashgate.

McKenzie, A. B. (2009). Axial compressor development at Rolls-Royce Derby, 19461962. Derby: Rolls-Royce Heritage Trust.

Miyake, Y., \& Fujita, S. (1974). A laminar boundary layer on a rotating three-dimensional blade. Journal of Fluid Mechanics, 65(3): 481-498. 
Moore, R. W., \& Richardson, D. L. (1955). A study of the end wall boundary layer in an axial compressor blade row. Cambridge, Massachusetts: Gas Turbine Laboratory, Massachusetts Institute of Technology Report.

Morris, P. J. (1981). The three-dimensional boundary layer on a rotating helical blade. Journal of Fluid Mechanics, 112: 283-296.

Nash, J. F., \& Hicks, J. G. (1971). The calculation of three-dimensional turbulent boundary layers on helicopter rotors. Marietta, Georgia: NASA Contractor Report prepared by Lockheed Georgia Research Laboratory.

Reynolds, B., \& Lakshminarayana, B. (1979). Characteristics of lightly loaded fan rotor blades. University Park, Pennsylvania: NASA Contractor Report prepared by Pennsylvania State University.

Smith, L. H. (1970). Casing boundary layers in multistage axial flow compressors. Flow research on blading, 106: 635-647.

Spalart, P. R., \& Allmaras, S. R. (1992). A one equation turbulence model for aerodynamic flows. 30th American Institute of Aeronautics and Astronautics Meeting and Exhibit. Reno, Nevada.

Stratford, B. S., Jawor, Z. M., \& Golesworthy, G. T. (1964). The mixing with ambient air of a cold stream in a centrifugal field. London, U.K: Ministry of Aviation, Aeronautical Research Council Report.

Taylor, G. I. (1932). Stability of a viscous liquid contained between two rotating cylinders. Philosophical Transactions of the Royal Society of London. Series A, Containing Papersof a Mathematical or Physical Character, 223: 289-343.

Taylor, J. V. (2016). Three-dimensional mechanisms in compressor flows. PhD thesis: University of Cambridge, U.K.

Taylor, J. V. (2019). Separated flow topology in compressors. Journal of Turbomachinery, 141(9): 091014.

Taylor, J. V., \& Miller, R. J. (2017). Competing three-dimensional mechanisms in compressor flows. Journal of Turbomachinery, 139(2): 021009. 
Taylor, J. V., Conduit, B., Dickens, A. M., Hall, C., Hillel, M., \& Miller, R. J. (2020). Predicting the operability of damaged compressors using machine learning. Journal of Turbomachinery, 142(5): 051010.

To, H. (2016). The effect of aspect ratio on compressor performance. $\mathrm{PhD}$ thesis: University of Cambridge, U.K.

To, H., \& Miller, R. J. (2019). The effect of aspect ratio on compressor performance. Journal of Turbomachinery, 141(8): 081011.

Wright, P. I., \& Miller, D. C. (1991). An improved compressor performance prediction model. European Conference of Turbomachinery: Latest Developments in a Changing Scene. London, U.K: IMechE paper C423/028.

Zierke, W. C., Farrell, K. J., \& Straka, W. A. (1994a). Measurements of the tip clearance flow for a high reynolds number axial-flow rotor Part I - flow visualization. ASME Turbo Expo Conference Proceedings. The Hague, Netherlands: ASME paper 94GT-453.

Zierke, W. C., Farrell, K. J., \& Straka, W. A. (1994b). Measurements of the tip clearance flow for a high reynolds number axial-flow rotor Part II - detailed flow measurements. ASME Turbo Expo Conference Proceedings. The Hague, Netherlands: ASME paper 94-GT-454. 



\section{Appendix A: Low order endwall loss model}

The total entropy generation, per unit pitch, in either the hub or casing endwall, can be calculated by:

$$
\dot{S}=L \int_{0}^{1} \frac{\mathrm{C}_{\mathrm{d}} \rho \mathrm{V}_{0}^{3}}{\mathrm{~T}} \mathrm{~d}\left(\frac{\mathrm{x}}{\mathrm{L}}\right)
$$

where $\mathrm{L}$ is the stage length and $\mathrm{V}_{0}$ is the boundary layer edge velocity. Writing equation A1 in the form of lost efficiency gives:

$$
\left(\frac{\mathrm{T} \Delta \mathrm{s}}{\Delta \mathrm{h}_{0}}\right)_{\text {endwall }}=2 \frac{\mathrm{C}_{0}}{\mathrm{~V}_{\mathrm{x}}} \frac{\mathrm{L}}{\Delta \mathrm{r}} \int_{0}^{1} \mathrm{C}_{\mathrm{d}}\left(\frac{\mathrm{V}_{0, \mathrm{ref}}}{\mathrm{C}_{0}}\right)^{3} \frac{\mathrm{dx}}{\mathrm{L}}
$$

where $\Delta \mathrm{r}$ is the blade span and $\mathrm{V}_{0 \text {,ref }}$ is the boundary layer edge velocity relative to the endwall. 



\section{Appendix B: Derivation of radial equilibrium equation}

Consider the balance of equilibrium of the small element of fluid in the compressor, illustrated in Figure B1. The balance of radial momentum at radius, r, gives:

$$
-\frac{1}{\rho} \frac{\mathrm{dp}}{\mathrm{dr}}=-\frac{\mathrm{V}_{\theta}^{2}}{\mathrm{r}}+\frac{\mathrm{V}_{\mathrm{m}}^{2}}{\mathrm{r}_{\mathrm{m}}} \cos (\phi)+\mathrm{V}_{\mathrm{m}} \frac{\mathrm{dV}_{\mathrm{m}}}{\mathrm{dm}} \sin (\phi)
$$

where $V_{m}$ is the meridional velocity, $r_{m}$ is the radius of the path of the fluid element in the meridional plane and $\phi$ is the angle between $V_{m}$ and the axial direction. The flow is assumed to be axisymmetric. The first term is the radial force per unit volume required to balance the radial components of acceleration, shown in Figure B1. If the compressor has a plane annulus, the meridional flow angle, $\phi$, is small and the meridional curvature of the fluid element along its path, $\mathrm{r}_{\mathrm{m}}$, is large. This means that the third and fourth terms in equation $\mathrm{B} 1$ can be dropped to give:

$$
\frac{1}{\rho} \frac{\mathrm{dp}}{\mathrm{dr}}=\frac{\mathrm{V}_{\theta}^{2}}{\mathrm{r}}
$$

which shows that the absolute tangential velocity $V_{\theta}$ sets up the radial static-pressure gradient. Integrating equation B2 shows clearly why the static-pressure p can never be 
lower at the casing than at the hub for the case of a plane annulus.For an incompressible flow the stagnation-pressure can be written as:

$$
\mathrm{p}_{0}=\mathrm{p}+\frac{1}{2} \rho\left(\mathrm{V}_{\mathrm{x}}^{2}+\mathrm{V}_{\theta}^{2}+\mathrm{V}_{\mathrm{r}}^{2}\right)
$$

which can be differentiated to give:

$$
\frac{1}{\rho} \frac{\mathrm{dp}_{0}}{\mathrm{dr}}=\frac{1}{\rho} \frac{\mathrm{dp}}{\mathrm{dr}}+\mathrm{V}_{\mathrm{x}} \frac{\mathrm{dV}_{\mathrm{x}}}{\mathrm{dr}}+\mathrm{V}_{\theta} \frac{\mathrm{dV}_{\theta}}{\mathrm{dr}}
$$

where the term corresponding to the rate of change, $d / d r$, of radial velocity, $V_{r}$, is not included as it is small relative to the remaining terms in equation B4.

Combining equations $\mathrm{B} 2$ and $\mathrm{B} 4$ gives the result:

$$
\frac{1}{\rho} \frac{\mathrm{dp}_{0}}{\mathrm{dr}}=\mathrm{V}_{\mathrm{x}} \frac{\mathrm{dV} \mathrm{V}_{\mathrm{x}}}{\mathrm{dr}}+\frac{\mathrm{V}_{\theta}}{\mathrm{r}} \frac{\mathrm{d}\left(\mathrm{rV}_{\theta}\right)}{\mathrm{dr}}
$$

which is a radial equilibrium equation for incompressible flow.

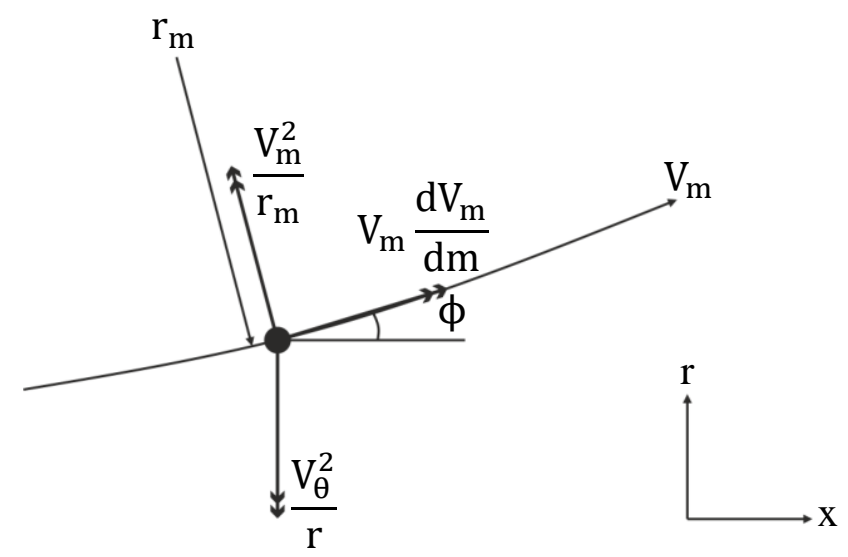

Figure B1: Components of acceleration for a small fluid element on an axisymmetric path. 